NBER WORKING PAPER SERIES

\title{
RULES WITHOUT COMMITMENT: REPUTATION AND INCENTIVES
}

\author{
Alessandro Dovis \\ Rishabh Kirpalani \\ Working Paper 26451 \\ http://www.nber.org/papers/w26451
NATIONAL BUREAU OF ECONOMIC RESEARCH
1050 Massachusetts Avenue
Cambridge, MA 02138 \\ November 2019
}

We thank Mark Aguiar, Gadi Barlevy, John Geanakoplos, Ben Hébert, Juanpa Nicolini, Erik Madsen, Ramon Marimon, Giuseppe Moscarini, Guillermo Ordonez, Chris Phelan, Facundo Piguillem, Aleh Tsyvinski, and Fabrizio Zilibotti for valuable comments. The views expressed herein are those of the authors and do not necessarily reflect the views of the National Bureau of Economic Research.

NBER working papers are circulated for discussion and comment purposes. They have not been peer-reviewed or been subject to the review by the NBER Board of Directors that accompanies official NBER publications.

(C) 2019 by Alessandro Dovis and Rishabh Kirpalani. All rights reserved. Short sections of text, not to exceed two paragraphs, may be quoted without explicit permission provided that full credit, including $\odot$ notice, is given to the source. 
Rules without Commitment: Reputation and Incentives

Alessandro Dovis and Rishabh Kirpalani

NBER Working Paper No. 26451

November 2019

JEL No. E6

\begin{abstract}
$\underline{\text { ABSTRACT }}$
This paper studies the optimal design of rules in a dynamic model when there is a time inconsistency problem and uncertainty about whether the policy maker can commit to follow the rule ex post. The policy maker can either be a commitment type, which can always commit to follow rules, or an optimizing type, which sequentially decides whether to follow rules or not. This type is unobservable to private agents, who learn about it through the actions of the policy maker. Higher beliefs that the policy maker is the commitment type (the policy maker's reputation) help promote good behavior by private agents. We show that in a large class of economies, preserving uncertainty about the policy maker's type is preferable from an ex-ante perspective. If the initial reputation is not too high, the optimal rule is the strictest one that is incentive compatible for the optimizing type. We show that reputational considerations imply that the optimal rule is more lenient than the one that would arise in a static environment. Moreover, opaque rules are preferable to transparent ones if reputation is high enough.
\end{abstract}

\author{
Alessandro Dovis \\ Department of Economics \\ University of Pennsylvania \\ The Ronald O. Perelman Center \\ for Political Science and Economics \\ 133 South 36th Street \\ Philadelphia, PA 19104 \\ and NBER \\ adovis@upenn.edu \\ Rishabh Kirpalani \\ Department of Economics \\ University of Wisconsin-Madison \\ Madison, WI 53706 \\ rishabh.kirpalani@wisc.edu
}




\section{Introduction}

Since Kydland and Prescott (1977), a large literature in macroeconomics has grappled with the problem of designing policies when there are time inconsistency problems. Rules are often proposed as a solution to the time inconsistency problem. The implicit assumption is that society can credibly impose rules on policy makers and that policy makers can commit to follow these rules. However, at the time when rules and regulations are formulated, there is often substantial uncertainty about whether policy makers can resist the temptation to deviate ex-post from the stated rules if it is optimal for them to do so. This uncertainty is only resolved over time as the actions of policy makers are observed. The combination of uncertainty and learning generates reputational incentives for policy makers.

The key question motivating this paper is how should rules be designed taking into account both the uncertainty about the policy makers' ability to follow the rules ex-post and their reputational building incentives. To do this we study the optimal design of policy rules in a dynamic game between policy makers and private agents in which the policy maker's ability to commit is private information. We define the public beliefs about the ability of the policy maker to commit as the policy maker's reputation. The main result of our paper is that if the initial reputation is low enough, the optimal rule should be designed to preserve uncertainty in future periods. This is implemented by introducing leniency in policy. In contrast, if the initial reputation is high, the optimal rule should promote learning about this type. We also show that designing opaque rules can be beneficial when reputation is high since they help preserve uncertainty without the need to introduce leniency in rules.

The insights from our theory can be applied to many relevant policy design questions including the design of central bank mandates, fiscal rules in federal governments, and financial regulation. Consider, for instance, the optimal design of financial regulation. As is well understood, in a large class of economies, if regulators can commit, a no-bailout policy is optimal in order to prevent excessive risk taking by financial institutions ex-ante. In particular, creditors should be forced to take losses in the event of default (bail-in). If the reputation of regulators is not sufficiently high, our analysis suggests that allowing for partial bailouts in equilibrium is optimal. We show that, contrary to conventional wisdom, bailouts along the equilibrium path are necessary to discipline future risk-taking of financial firms as they preserve uncertainty about the type of the policy maker.

We consider a dynamic model with three types of agents: a rule designer, policy makers, and private agents. The rule designer chooses a rule, which consists of a policy recommendation to policy makers, in order to maximize the expected social welfare. After the rule is chosen, the private agents take their actions and, finally, the policy maker chooses 
a policy. As in Barro (1986), the policy maker can be one of two types: a commitment type, which always follows the recommendation, or an optimizing type, which follows the recommendation only if it is sequentially optimal to do so. This type is unobservable to both the rule designer and private agents. We define the beliefs that the policy maker is the commitment type as its reputation.

We present two leading examples of our framework. The first is a model similar to Barro and Gordon (1983b) in which the rule designer must choose the optimal inflation target. The second is a banking model in the spirit of Kareken and Wallace (1978) where there is a trade-off between providing incentives to bankers for taking appropriate levels of risk ex-ante and bailing them out ex-post to avoid a costly default. In this case the rule designer chooses an optimal bailout policy.

We first study a static problem. Since there is no way to incentivize the optimizing type to choose any policy other than the ex-post optimal one, the best the rule designer can do is get the commitment type to follow the Ramsey policy. We show that under certain conditions, uncertainty is beneficial in that the expected social welfare is higher when the private agents and the rule designer are uncertain about the type of the policy maker relative to the case in which this type is revealed right before the rule designer chooses the rule. That is, the rule designer's static value is concave in the policy maker's reputation. ${ }^{1}$ This is because under our assumptions there are decreasing returns to reputation. In the context of the bailout example, an increase in reputation incentivizes banks to take on less risk, and the disciplining effect of reputation is greater when reputation is low and banks are taking on a lot of risk.

We then consider a repeated version of this policy game. Unlike in the static model, the optimizing type now cares about its reputation in the following period as it affects the actions of the private agents. Thus, it can be incentivized to choose policies other than its static best response. We show that when reputation is low, the rule designer wants to preserve uncertainty about the type of the policy maker. The optimal rule in this case is the most stringent policy that is incentive compatible for the optimizing type. This recommended policy is more lenient than the statically optimal one. Leniency in the rule makes it easier for the optimizing type to follow the recommendation ex-post. This has dynamic benefits because it prevents the private agents from learning the type of the policy maker, and uncertainty is beneficial. When reputation is low, inducing the optimizing type to follow the rule also has static benefits. This is because it promotes better behavior by the private agents who anticipate that the optimizing type will follow

\footnotetext{
${ }^{1}$ Nosal and Ordoñez (2016) also consider an environment in which uncertainty can mitigate the time inconsistency problem. The mechanism is very different: here there is uncertainty about the policy maker's type, while in their paper there is uncertainty about the state of the economy, which restrains the policy maker ex-post.
} 
the rule - albeit more lenient - instead of the statically optimal policy.

This result has sharp implications for policy. In the context of optimal inflation targeting, having looser inflation targets is beneficial when reputation is low. Another application of our framework is the design of exchange rate regimes. Our result suggests that when reputation is low, crawling pegs might be superior to fixed exchange rate policies. Similarly, in the context of financial regulation, if reputation is low, the optimal rule is not a strict no-bailout policy that imposes losses on lenders. By explicitly allowing for partial bailouts along the equilibrium path, the rule designer makes it easier for the optimizing type to adhere to the rule and maintain its reputation. The optimal rule prescribed by the model is in contrast with the observed design of financial regulation after the 2008 financial crisis. After the bailouts of financial institutions during this crisis, the reputation of regulators was arguably low. While our model prescribes a more lenient bailout policy in this situation, the Dodd-Frank Act imposed very strict no-bailout policies.

In contrast, if reputation is sufficiently high, the rule designer finds it optimal to set stringent rules that result in the type of the policy maker being revealed. This is because when reputation is sufficiently high, there are static costs associated with choosing a lenient rule. In this case, the private agents anticipate that the rule will be followed with sufficiently high probability and so by choosing the Ramsey policy, the rule designer can obtain a value close to the Ramsey outcome. There are, however, dynamic losses associated with choosing the Ramsey policy: if the rule is to follow the Ramsey policy, for a low enough discount factor, the optimizing type will not follow the rule and there will be revelation about the type of the policy maker in the first period. Because uncertainty is beneficial, the expected continuation value is lower than in the case in which the type of the policy maker is not revealed. When reputation is high enough, the static benefits of choosing a stringent rule outweigh the dynamic losses.

We then show that the rule designer itself suffers from a time-inconsistency problem. In particular, we study the problem for a rule designer who can choose rules for each subsequent period in period 0 and commit to them. We show that the solution to this problem is different than the baseline in which the rule designer chooses the rule each period. This is because the rules in period $t+1$ can provide incentives to the policy maker in period $t$ which are not internalized by the rule designer in period $t+1$. In particular, we show that the prospect of stringent rules in period $t+1$ provides more incentives to the optimizing type in period $t$. Thus, for a range of prior reputation levels, the rule designer in period $t$ would like to choose a stringent rule in period $t+1$ that induces separation, while the rule designer in period $t+1$ would like to choose a lenient rule that induces pooling.

Next, we study the optimal degree of transparency of the rule. We say that a rule is transparent if the policy maker's deviations are easily detectable. In repeated policy 
games with no reputational considerations, perfect monitoring is always desirable. See Atkeson and Kehoe (2001), Atkeson et al. (2007), and Piguillem and Schneider (2013). In contrast, we show that with reputational considerations, transparent rules are desirable only for low levels of reputation, while opaque rules are desirable for high levels of reputation. ${ }^{2}$ This is because they can help maintain reputation without the static costs associated with pooling when reputation is high.

We consider two ways in which the rule designer can affect the transparency of the rules. First, we assume that future private agents and rule designers observe only a signal of the chosen policy and the rule designer can choose the precision of the signal. High precision (transparency) is beneficial because it incentivizes the optimizing type to follow the rule, as a deviation results in the revelation of its type with large reputation losses. Low precision (opaqueness) is beneficial because it allows the rule designer to maintain uncertainty about the policy maker's type. For instance, if the signals are imprecise, the private agents attribute the observed deviations from the stated policy to noise rather than to the policy maker being the optimizing type that deviated from the policy. This is helpful for high levels of reputation since the rule designer would like to choose the Ramsey policy from a static perspective. As discussed earlier, there is a trade-off between the static value of having the commitment type follow a stringent rule and the dynamic losses associated with learning the policy maker's type. Allowing for opaque rules helps break this trade-off: the rule designer can achieve both the high static pay-off of choosing a rule equal to the Ramsey policy without the costs associated with separation for sure because the policy observations are very noisy. A similar argument implies that it is optimal to have short tenure for the policy maker when reputation is high.

An alternative way of introducing opacity in rules is to allow the rule designer to choose stochastic rules even though fundamentals are deterministic. When reputation is low, the optimal rule has no randomization in order to maximize the incentives of the optimizing type to follow more stringent policies. When reputation is high instead, it is optimal to have randomization in order to reduce the dispersion in the posteriors.

In our baseline setup, we model the commitment type as a policy maker that cannot deviate from the rules. One interpretation of this is that the commitment type suffers a cost from deviating from the stated rule over and above the reputational cost in the model. For example, a deviation may affect the commitment's type ability to be elected to higher offices, while the optimizing type may not have such ambitions. Alternatively, one could assume that policy makers are identical, but there is uncertainty about whether these policy deviations can be enacted, due to legislative holdups, for example. In particular, policy makers always have an incentive to choose policies which are sequentially rational,

\footnotetext{
${ }^{2}$ In the principal-agent literature there are examples of environments where imperfect monitoring is beneficial to provide incentives. See for instance Crémer (1995) and Prat (2005).
} 
but might face roadblocks in implementation if the legislature is controlled by opponents who might block these policies for purely political purposes. As in Piguillem and Riboni (2018), the rule can be the default option in case of such disagreements. In this case, we can interpret the commitment type as a policy maker which faces such roadblocks, and the optimizing type as one which does not. The latter might want to pretend as if its hands are tied (like the commitment type) for exactly the same reasons as in the baseline model.

An alternative approach is to assume that the two types of policy makers differ in their preferences (payoff types). For example, policy makers can differ in their temptation to deviate ex-post because certain policy makers can better resist the pressure from interest groups ex-post or simply have different preferences over outcomes than the social welfare function, as in the seminal Rogoff (1985) paper. The outcomes in this case differ from the ones in the baseline model: we show that with preference types and a reasonable belief refinement, the equilibrium with payoff types has separation for all levels of initial reputation.

Related literature This paper is related to the literature that studies the trade-off between rules and flexibility. See for example Athey et al. (2005), Halac and Yared (2014), Halac and Yared (2017), and Azzimonti et al. (2016), among others. The focus of this literature is on how much flexibility to leave the policy maker when it is not possible to make the rule contingent on the state of the economy (say because it is private information to the policy maker). We abstract from this issue by considering a deterministic environment, but we focus instead on the uncertainty about the ability of the policy maker to commit. Our paper is also related to the literature that studies optimal policies without commitment when it is known that the policy maker cannot commit. This is the approach followed by a large literature on time consistent policies, including Barro and Gordon (1983b), Chari and Kehoe (1990), Phelan and Stacchetti (2001), and Halac and Yared (2018). Our paper nests simple versions of these two approaches as special cases when reputation is either one or zero.

This paper builds on the reputation literature that originates with Milgrom and Roberts (1982) and Kreps and Wilson (1982). See Barro (1986), Backus and Driffill (1985), Phelan (2006), Amador and Phelan (2018), and Dovis and Kirpalani (2019b) for recent applications to policy games. Most of this literature takes as given the policy chosen by the commitment type and analyses the incentives of the optimizing type and the outcomes that can be achieved. The goal of this paper is to study the optimal policy that the commitment type should follow.

A key driver of our results is the idea that uncertainty about the policy maker type is beneficial. This feature is also present in Dovis and Kirpalani (2019a). Our contribu- 
tion is to show how this property affects the design of the optimal rule. Marinovic and Szydlowski (2019), Bond and Zeng (2018), and Asriyan et al. (2019) also consider environments in which uncertainty is beneficial and it is not optimal to resolve uncertainty. In these models, the focus is on whether the agent having the information should disclose it to the other agent(s) in the economy. In contrast, the rule designer in our model does not know the policy maker's type and we focus on the design of policies that can induce - or not - revelation. In Section 5.1, we consider an environment in which the rules are chosen by the policy makers (who know their type) instead of the rule designer and show that the results are very different. In particular, for intermediate levels of discount factors, there will be separation for all priors.

Our paper is also related to a literature that studies signaling games when policy makers have different types. See for instance Vickers (1986), Cole et al. (1995), Angeletos et al. (2006), King et al. (2008), Lu (2013), and Lu et al. (2016) with payoff types, or Dovis and Kirpalani (2019a), where one type has the ability to commit to the announced policy. See also Sanktjohanser (2018) for a similar analysis in the context of a bargaining game. Our approach differs from these papers since we study the best policy chosen by the rule designer when there is uncertainty about the type of the policy maker, while these papers study the optimal policy that the commitment type would choose knowing its type. We show that if the rules are chosen by the policy maker, the commitment type (if sufficiently patient) chooses a stringent rule to separate from the optimizing type for all levels of reputation, while the rule designer under the veil of uncertainty chooses to avoid separation when the reputation of the policy maker is sufficiently low.

Debortoli and Nunes (2010) consider a policy game in which the policy maker has the ability to change its policies infrequently and randomly. They abstract from reputationbuilding incentives.

\section{Policy game}

We consider a policy game that captures a variety of relevant economic environments as special cases. We present two leading examples of our framework: a version of the Barro and Gordon (1983a) model of monetary policy and a banking model in the spirit of Kareken and Wallace (1978). Our framework also nests other models, including the Fisher model of capital income taxation considered in Chari and Kehoe (1990).

There are three types of agents: the rule designer, policy makers (or bureaucrats), and a continuum of private agents. We consider a repeated environment where there are no endogenous state variables across periods. At the beginning of each period, the rule designer recommends a policy $\pi_{\mathrm{r}}$ from a set $[\underline{\pi}, \bar{\pi}]$. We refer to this recommendation as 
a rule. The private agents then choose an individual action. After observing the private action, the policy maker chooses a policy $\pi$. The policy maker can be one of two types: a commitment type, which always follows the recommendation made by the rule designer, or an optimizing type, which can choose any policy $\pi$ in the set $[\pi, \bar{\pi}]$. We assume that the policy maker's type is permanent. ${ }^{3}$ The policy maker's type is unobservable to the private agents and the rule designer, who learn about it through the observed policies. We assume that the private agents and the rule designer share a common prior $\rho$ that they are facing the commitment type. We define the probability that the private agents and the rule designer ascribe to the policy maker being the commitment type as the policy maker's reputation.

We let $x$ denote the representative (average) action taken by the private agents. We assume that the private action is a function $\phi$ of the expected policy, $\mathbb{E} \pi=\rho \pi_{c}+(1-\rho) \pi_{0}$, where $\pi_{\mathrm{c}}=\pi_{\mathrm{r}}$ is the policy chosen by the commitment type and $\pi_{\mathrm{o}}$ is the policy implemented by the optimizing type,

$$
x=\phi(\mathbb{E} \pi) .
$$

We will refer to (1) as the implementability constraint. We think of the function $\phi$ as summarizing the set of implementability conditions describing the set of outcomes that can be implemented given a set of policies or an incentive compatibility constraint.

The rule designer and the policy makers maximize a social welfare function $w(x, \pi)$. We assume that the problem is time inconsistent. Specifically, we define the Ramsey outcome as

$$
\left(x_{\text {ramsey }}, \pi_{\text {ramsey }}\right)=\arg \max _{x, \pi} w(x, \pi) \quad \text { subject to } \quad x=\phi(1, \pi) .
$$

We assume that there is a time-inconsistency problem in that the Ramsey policy is not optimal ex-post, i.e., $\pi_{\text {ramsey }} \neq \pi^{*}\left(x_{\text {ramsey }}\right)$, where $\pi^{*}(x)$ denotes the best response of the government to $x, \pi^{*}(x)=\arg \max _{\pi} w(x, \pi)$. We assume without loss of generality that $\pi^{*}\left(x_{\text {ramsey }}\right)>\pi_{\text {ramsey }}$.

We also make the following assumptions about $w$ and $\phi$ :

\section{Assumption 1. Assume that}

1. If $w_{x}>0$ then $\phi^{\prime} \leqslant 0, \phi^{\prime \prime} \leqslant 0$, and $w_{x \pi}<0$

2. If $w_{x}<0$ then $\phi^{\prime} \geqslant 0, \phi^{\prime \prime} \geqslant 0$, and $w_{x \pi}>0$.

As is standard in the time-inconsistency literature, we consider environments in which the inability of the policy maker to commit ex-post incentivizes the private agents to take

\footnotetext{
${ }^{3}$ This assumption is made for convenience. Our main results extend to the case in which the policy maker's type can change exogenously, provided that this type process is persistent. When types are i.i.d., there is no role for reputation.
} 
worse actions ex-ante. Thus, if social welfare is increasing in the private action $x$, we assume that if the agents expect higher $\pi$, they choose lower values of $x\left(\phi^{\prime} \leqslant 0\right)$. We also assume that the private action $x$ is concave in expected policy. Finally, we assume a form of supermodularity in $(x, \pi)$ which implies that the government's incentive to deviate from its ex-ante promises is higher the worse the private action (low $x$ ) is.

We next present two economies and show how they map into our general framework.

Example 1: Barro-Gordon One special case of the general environment is the classic Barro and Gordon (1983a) model used to analyze the time inconsistency problem in monetary policy. In this context, we interpret $x$ as the average wage inflation, and $\pi$ is the money growth rate (or price inflation).

We assume that the private agents set wage inflation according to

$$
\chi=\phi(\mathbb{E} \pi)=\rho \pi_{c}+(1-\rho) \pi_{\mathrm{o}}
$$

The social welfare function takes the quadratic form

$$
w(x, \pi)=-\frac{1}{2}\left[(\psi+x-\pi)^{2}+\pi^{2}\right]
$$

with $\psi>0$. The first term in this functional form represents the welfare losses associated with low employment due, for example, to monopolistic competition in labor markets. The parameter $\psi$ measures the extent of this distortion, and it can be mapped into the wage markup set by unions. The second term captures the costs of ex-post inflation (due, for example, to the transactional value of real money balances).

Example 2: Bailout and effort We now consider another economy inspired by the classic analysis in Kareken and Wallace (1978), which studies the trade-off between the expost benefits and the ex-ante costs of bailouts.

There are two types of private agents: depositors and bankers. At the beginning of each period, the banker must borrow $k=1$ from the depositors to finance an investment opportunity that pays off at the end of the period. The return of the investment opportunity is $R_{H}$ with probability $p(e)$, where $e$ is the effort exerted by the banker, and 0 with probability $1-p(e)$. The function $p$ is increasing and concave, and it satisfies the Inada conditions. Exerting effort e results in a utility $\cos t v(e)$, where $v$ is increasing and convex. We interpret the effort as the costs associated with monitoring the investment project. The bankers and the depositors are risk-neutral and do not discount consumption between the beginning and the end of the period.

The banker offers the depositors a contract that promises to repay $R$ units of the con- 
sumption good in the second sub-period subject to limited liability. We assume that society faces bankruptcy costs $\psi$ whenever the lenders recover less than their initial investment. ${ }^{4}$ The policy maker can avoid these bankruptcy costs by making a transfer to the banker to enable him to repay the depositors. In particular, the government can choose the recovery $\pi$ in case the banker is unable to repay. There is a taxation cost associated with these transfers, denoted by $c(\pi)$, where $c$ is increasing and convex. To simplify calculations we assume that $p(e)=e^{\alpha}, v(e)=e^{2} / 2$, and $c(\pi)=\lambda \pi^{2} / 2$. We assume that if the recovery is $\pi$, the bankruptcy costs are $\psi(1-\pi)$.

We assume that the depositors can observe the effort $e$. They are then willing to lend to the banker if the interest rate is at least

$$
R(e)=\frac{1-(1-p(e))\left[\rho \pi_{c}+(1-\rho) \pi_{0}\right]}{p(e)} .
$$

The banker chooses the effort to maximize $-v(e)+p(e)\left[R_{H}-R(e)\right]$ subject to (2). Using (2) to substitute for $R(e)$, we can rewrite the banker's problem as

$$
\max _{e}-v(e)+p(e) R_{H}+(1-p(e)) \mathbb{E} \pi,
$$

where the term $(1-p(e)) \mathbb{E} \pi$ represents the distortion induced by the expected bailout. Thus the optimal effort $e$ is a function $\phi(\mathbb{E} \pi)$ that is implicitly defined by the first order condition

$$
v^{\prime}(e)=p^{\prime}(e)\left[R_{H}-\mathbb{E} \pi\right] .
$$

The social welfare function is the equally weighted sum of the utility of the bankers and depositors net of taxation and bankruptcy costs:

$$
w(e, \pi)=-v(e)+p(e) R_{H}-1-(1-p(e))(1-\pi) \psi-c(\pi) .
$$

\section{Optimal rules}

We now consider the problem of how to design the optimal rule. We begin by characterizing the rule designer's problem in a static setting and next study how the optimal rule changes once we introduce dynamics. We first establish a set of sufficient conditions under which uncertainty about the policy maker's type is beneficial in the static economy. Our main result is that when reputation is low, the rule designer designs a rule which preserves uncertainty about the type of the policy maker. The optimal rule is the most stringent policy that is incentive compatible for the optimizing type. This recommended

\footnotetext{
${ }^{4}$ Alternatively, we could have assumed that these costs are incurred whenever the lenders recover less than the promised return.
} 
policy is more lenient than the statically optimal one. Leniency in the rule makes it easier for the optimizing type to follow the recommendation ex-post. In contrast, if reputation is sufficiently high, the rule designer finds it optimal to set stringent rules that result in the type of the policy maker being revealed. This is because when reputation is sufficiently high, the static costs associated with choosing a lenient rule outweigh the benefits associated with preserving uncertainty about the policy maker's type.

We next relax the assumption that the rule designer chooses a rule in each period. First, we show that the rule designer itself suffers from a time inconsistency problem and contrast the optimal path of rules chosen in period zero with the ones chosen sequentially. Second, we consider a set-up in which the rule designer lacks commitment but is only stochastically able to change the rule each period.

\subsection{Statically optimal rules}

We begin by studying the optimal rule in a static setting. The rule designer anticipates that if the policy maker is the commitment type, it will follow the rule $\pi_{\mathrm{r}}$. Instead, if the policy maker is the optimizing type, it will always choose the static best response to the private action $x$. This is because in a static model the rule designer has no tools to incentivize the optimizing type to take any other action. Of course, this will change in the dynamic setting.

We can then write the problem for the rule designer as choosing the recommendation for the commitment type, $\pi_{c}$, to solve

$$
W_{0}(\rho)=\max _{\pi_{\mathbf{c}}} \rho w\left(x, \pi_{c}\right)+(1-\rho) w\left(x, \pi_{\mathrm{o}}\right),
$$

where given $\pi_{\mathrm{c}}$ and the prior $\rho, \mathrm{x}$ and $\pi_{\mathrm{o}}$ are given by

$$
\begin{aligned}
x & =\phi\left(\rho \pi_{c}+(1-\rho) \pi_{0}\right) \\
\pi_{0} & =\pi^{*}(x) .
\end{aligned}
$$

For later reference, we denote the solution to this problem as $\pi_{\mathrm{c} 0}(\rho), \pi_{\mathrm{o} 0}(\rho)$, and $x_{0}(\rho) .^{5}$ We can also define the value for the optimizing type:

$$
V_{0}(\rho)=w\left(x_{0}(\rho), \pi^{*}\left(x_{0}(\rho)\right)\right) .
$$

\footnotetext{
${ }^{5}$ Note that here we are allowing the rule designer to choose the best equilibrium given a rule $\pi_{\mathrm{r}}$. Thus there is no need to have the rule depend on the representative private action $x$.
} 
We next discuss the conditions under which uncertainty is beneficial in that

$$
W_{0}(\rho) \geqslant \rho W_{0}(1)+(1-\rho) W_{0}(0)
$$

When uncertainty is beneficial, the expected social welfare is higher when the policy maker's type is uncertain relative to the case in which types are revealed right before the rule designer chooses the rule. This property of the static problem turns out to be critical for the form of the optimal rule in a dynamic model. ${ }^{6}$

We now provide a set of sufficient conditions on primitives that ensure that uncertainty is beneficial. In the appendix we show that the Barro-Gordon and bailout examples satisfy these assumptions.

\section{Assumption 2. Assume that}

1. $w(x, \pi)$ is concave in $(x, \pi)$.

2. $w_{\pi}(x, \pi)$ is convex in $(x, \pi)$.

3. $1>\pi^{*}{ }_{x}(x) \phi^{\prime}(\pi) \geqslant 1-\frac{w_{x}\left(\underline{x}, \pi^{*}(\underline{x})\right)}{w_{x}(\underline{x}, \underline{\pi})}$, where $x=\phi(\pi), \underline{x}=\phi(\underline{\pi})$, and $\pi_{x}^{*}(x)=$ $-\frac{w_{x \pi}\left(x, \pi^{*}(x)\right)}{w_{\pi \pi}\left(x, \pi^{*}(x)\right)}$.

4. $w_{\pi}(x, \pi)+\left[\rho w_{\chi}(x, \pi)+(1-\rho) w_{x}\left(x, \pi^{*}(x)\right)\right] \frac{\phi^{\prime}(\cdot)}{\left[1-\phi^{\prime}(\cdot)(1-\rho) \pi_{x}^{*}(x)\right]} \leqslant 0$ for all $\pi$.

We have the following lemma:

Lemma 1. Under Assumptions 1 and $2, \pi_{\mathrm{c} 0}(\rho)=\underline{\pi}$ and uncertainty is beneficial in that (5) holds.

The optimal static rule takes a simple form: for all $\rho$, the rule is set to $\pi_{\mathfrak{c}}(\rho)=\pi$, which is also the Ramsey policy. ${ }^{7}$ This result follows from Condition 4 in Assumption 2. The expression in Condition 4 is the first-order condition for the problem in (3). The condition implies that reducing $\pi$ has a positive marginal effect and thus it is optimal to be at the corner $\underline{\pi}$. Therefore, it is optimal for the rule designer to recommend the most stringent possible policy. In the context of the Barro-Gordon example, this means that the optimal inflation target is zero, while in the bailout example, a strict no-bailout policy is optimal.

We now argue that under our assumptions, condition (5) holds. To establish that uncertainty is beneficial it is sufficient to show that $W_{0}(\rho)$ is concave or equivalently that there are decreasing returns to reputation. To understand why, consider an increase in

\footnotetext{
${ }^{6}$ In Appendix B we provide an example of an environment that does not satisfy this property.

${ }^{7}$ This is true even though the private action is not at the Ramsey level since the private agents anticipate that with probability $1-\rho$ the policy maker is the optimizing type who will deviate from the recommendation and choose the static best response.
} 
reputation $\rho$. We illustrate the logic for the case in which $w_{x}>0$, as in the bailout model. A specular logic holds for the case in which $w_{x}<0$. First, notice that an increase in $\rho$ increases $x_{0}(\rho)=\phi\left(\rho \underline{\pi}+(1-\rho) \pi^{*}\left(x_{0}(\rho)\right)\right)$ through a direct channel (since $\underline{\pi}<\pi^{*}$ and $\phi$ is decreasing) and an indirect channel since $\pi^{*}$ is decreasing in $x$, which in turn increases $x$ further. Since $\phi$ is concave (Assumption 1) and $\pi^{*}$ is convex (which follows from Condition 2 of Assumption 2), the increase in $x_{0}$ will be larger for low $\rho$ than for high $\rho$. Consequently, $x_{0}$ is concave in $\rho$. Next, the concavity of $w$ and the concavity of $x_{0}$ imply that $w\left(x_{0}(\rho), \underline{\pi}\right)$ and $w\left(x_{0}(\rho), \pi_{0}(\rho)\right)$ are concave in $\rho$. Establishing the concavity of $w\left(x_{0}(\rho), \underline{\pi}\right)$ and $w\left(x_{0}(\rho), \pi_{0}(\rho)\right)$ is not enough to show that $W_{0}(\rho)=\rho w\left(x_{0}(\rho), \underline{\pi}\right)+(1-\rho) w\left(x_{0}(\rho), \pi_{0}(\rho)\right)$ is concave since the product of two concave functions is not necessarily concave. However, the technical assumption in Condition 3 guarantees that $W_{0}(\rho)$ is concave.

Intuitively, the marginal value of reputation is larger when reputation is low. In the context of the bailout example, this implies that an increase in reputation will incentivize banks to increase their effort by more when reputation is low than when reputation is high, i.e., $x_{0}$ is concave in $\rho$, as shown in Figure 1. In other words, the disciplining effect of reputation is greater when reputation is low and banks are exerting little effort.

Figure 1: Static value and private action when $w_{x}>0$
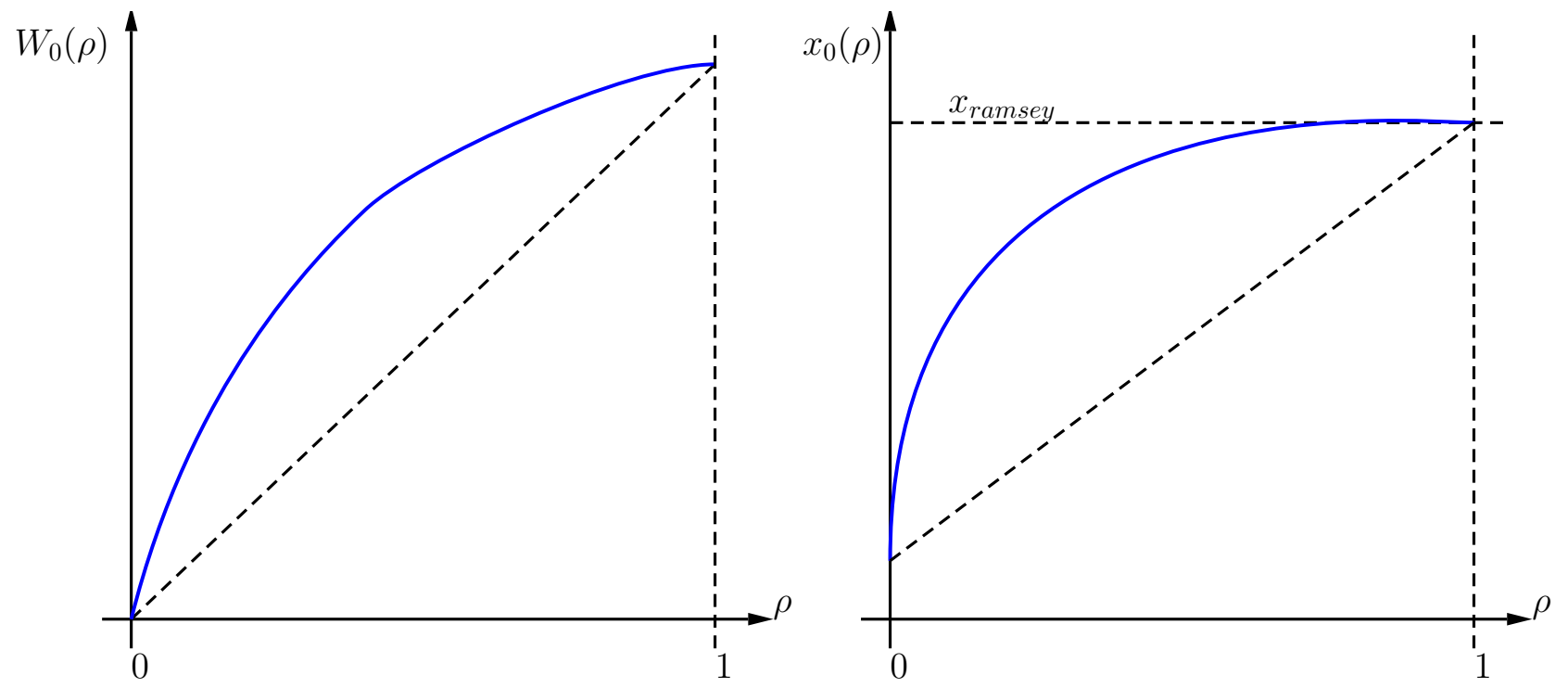

\subsection{Dynamic problem}

We now study the optimal rule design problem in a dynamic setting. We start by repeating the stage game, studied in the previous section, twice and then analyze what happens as the number of periods goes to infinity. 
When there is more than one period, the optimizing type can be incentivized to take a different action from its static best response. We can set up the rule designer's problem as choosing the rule that will be followed by the commitment type, $\pi_{r}=\pi_{c}$, and a recommendation to the optimizing type, $\pi_{0}$. This recommendation must be incentive compatible in that the optimizing type must prefer to follow the recommendation than to choose its best possible deviation (playing the static best response $\pi^{*}(x)$ ) and attaining a continuation value $V_{0}(0)$ as the prior jumps to zero:

$$
w\left(x, \pi_{\mathrm{o}}\right)+\beta_{\mathrm{o}} \mathrm{V}_{0}\left(\rho^{\prime}\left(\pi_{\mathrm{o}}\right)\right) \geqslant w\left(x, \pi^{*}(x)\right)+\beta_{\mathrm{o}} \mathrm{V}_{0}(0)
$$

where $\beta_{0}$ is the discount factor for the optimizing type and $\rho^{\prime}\left(\pi_{0}\right)$ is the private belief about the policy maker's type after observing $\pi_{\mathrm{o}}$ (given recommendation $\pi_{\mathrm{c}}$ ). ${ }^{8}$ The law of motion for beliefs on path follows Bayes' rule

$$
\rho^{\prime}(\pi)=\left\{\begin{array}{ll}
\frac{\rho}{\rho+(1-\rho) \sigma} & \text { if } \pi=\pi_{\mathrm{c}} \\
0 & \mathrm{o} / \mathrm{w}
\end{array},\right.
$$

where $\sigma$ is an indicator variable that takes value 1 if the private agents expect the optimizing type to choose the same policy as the commitment type, $\pi_{\mathrm{o}}=\pi_{\mathrm{c}}$, and $\sigma=0$ otherwise.

For all subsequent analyses we assume that the policy makers are sufficiently impatient so that the Ramsey outcome is not incentive compatible:

Assumption 3. The discount factor $\beta_{\mathrm{o}}$ is small enough so that

$$
w\left(x_{\text {ramsey }}, \pi^{*}\left(x_{\text {ramsey }}\right)\right)-w\left(x_{\text {ramsey }}, \pi_{\text {ramsey }}\right)>\frac{\beta_{\mathrm{o}}}{1-\beta_{\mathrm{o}}}\left[\mathrm{V}_{0}(1)-\mathrm{V}_{0}(0)\right]
$$

We can then write the rule designer's problem as

$$
W(\rho)=\max _{x, \pi_{c}, \sigma} \rho\left[w\left(x, \pi_{c}\right)+\beta W_{0}\left(\rho^{\prime}\left(\pi_{c}\right)\right)\right]+(1-\rho)\left[w\left(x, \pi_{0}\right)+\beta W_{0}\left(\rho^{\prime}\left(\pi_{0}\right)\right)\right]
$$

subject to the implementability condition,

$$
x=\phi\left(\rho \pi_{c}+(1-\rho)\left[\sigma \pi_{c}+(1-\sigma) \pi^{*}(x)\right]\right),
$$

the incentive compatibility constraint for the optimizing type (6), and the law of motion

\footnotetext{
${ }^{8}$ If the rule designer chooses $\pi_{\mathrm{o}}=\pi_{\mathrm{c}}$, then a deviation only happens off-path and thus Bayes' rule does not pin down the posterior. On the right side of (6), we assume that after a deviation, the posterior goes to zero. This is reasonable because the commitment type cannot deviate. Moreover, it also constitutes the worst punishment in the event that the optimizing type deviates.
} 
for beliefs (7). Note that we allow in principle for the rule designer's discount factor $\beta$ to differ from $\beta_{0}$, although this is not critical.

For simplicity we abstract from mixed strategies for the optimizing type. In Appendix A.2, we show that this is without loss of generality. Under our assumptions, the outcome in which the optimizing type follows the rule with probability $\sigma \in(0,1)$ and the expost optimal policy with probability $1-\sigma$ is dominated in terms of welfare by the best equilibrium in which the optimizing type follows the rule with probability one. This is because of two reasons. First, since uncertainty is beneficial and the posterior is a martingale, mixing introduces volatility in the posterior without affecting its mean, which lowers the continuation value. Second, we show in the appendix that mixing tightens the optimizing type's incentive constraint because $w$ is concave in $\pi$ and $V_{0}$ is concave in $\rho$ and thus reduces static payoffs.

We can then reduce the problem above to a discrete choice between two options: separating or pooling. If the rule designer chooses to separate, it chooses the best static rule. Because of Assumption 3, the Ramsey outcome is not incentive compatible and the optimizing type will choose the static best response and not follow the rule so the type of the policy maker is revealed at the end of the period. Thus the continuation value is either $W_{0}(1)$ with probability $\rho$ or $W_{0}(0)$ with probability $1-\rho$. The value of separating is then

$$
W_{\text {sep }}(\rho)=W_{0}(\rho)+\beta\left[\rho W_{0}(1)+(1-\rho) W_{0}(0)\right]
$$

If the rule designer chooses to pool, it sets the rule to $\pi_{1, i c o}(\rho)$, which is the most stringent policy $\pi$ consistent with the incentive compatibility constraint for the optimizing type:

$$
w\left(\phi\left(\pi_{1, \text { ico }}(\rho)\right), \pi_{1, \text { ico }}(\rho)\right)+\beta V_{0}(\rho)=w\left(\phi\left(\pi_{1, \text { ico }}(\rho)\right), \pi^{*}\left(\phi\left(\pi_{1, \text { ico }}(\rho)\right)\right)\right)+\beta V_{0}(0)
$$

In this case, both types of policy makers follow the rule in equilibrium and thus uncertainty about the type is preserved and the continuation value is $W_{0}(\rho)$. The value of pooling is then

$$
W_{\text {pool }}(\rho)=\mathcal{W}\left(\phi\left(\pi_{1, \text { ico }}(\rho)\right), \pi_{1, \text { ico }}(\rho)\right)+\beta W_{0}(\rho)
$$

The next proposition shows that designing a rule that preserves uncertainty about the policy maker's type is valuable when reputation is low:

Proposition 1. Under Assumptions 1-3,

1. For $\rho$ close to one there is separation with probability one and $\pi=\pi_{0 c}(\rho)=\underline{\pi}$;

2. For $\rho$ close to zero there is pooling $(\sigma=1)$ and $\pi_{c}(\rho)>\pi_{0 c}(\rho)=\underline{\pi}$. 
In particular, for the Barro-Gordon economy, the optimal regulation has a cutoff property in that there exists a $\rho_{1}^{*} \in(0,1)$ such that:

1. For $\rho>\rho_{1}^{*}$ it is optimal to separate and $\pi=\pi_{0 c}(\rho)=\underline{\pi}$.

2. For $\rho \leqslant \rho_{1}^{*}$ it is optimal to pool and $\pi_{\mathfrak{c}}(\rho)=\pi_{1, \text { ico }}(\rho)>\pi_{0 \mathrm{c}}(\rho)=\underline{\pi}$.

The key implication of this proposition is that in contrast to the static case, when reputation is low, the rule designer recommends more lenient rules in order to preserve uncertainty about the policy maker's type in the future. To see why this is indeed the case, consider

$$
W_{\text {pool }}(\rho)-W_{\text {sep }}(\rho)=\Delta \omega(\rho)+\beta \Delta \Omega(\rho),
$$

where $\Delta \Omega(\rho) \equiv W_{0}(\rho)-\left[\rho W_{0}(1)+(1-\rho) W_{0}(0)\right]$ are the dynamic benefits of pooling and $\Delta \omega(\rho)$ are the static benefits of pooling given by

$$
\Delta \omega(\rho) \equiv w\left(\phi\left(\pi_{1, \text { ico }}(\rho)\right), \pi_{1, \text { ico }}(\rho)\right)-W_{0}(\rho)
$$

The dynamic and static benefits of pooling are plotted in Figure 2. Since uncertainty is beneficial, we know that $\Delta \Omega(\rho)>0$ for all $\rho \in(0,1)$ and equal to zero when there is no uncertainty and $\rho \in\{0,1\}$. Also by construction, the static benefits of pooling are zero for $\rho=0$, since $\pi_{1, \text { ico }}(0)=\pi_{00}(0)=\pi^{*}\left(x_{0}(0)\right)$, and negative for $\rho=1$, since $W_{0}(1)$ attains the Ramsey value and $w\left(\phi\left(\pi_{1, \text { ico }}(\rho)\right), \pi_{1, \text { ico }}(\rho)\right)<W_{\text {ramsey }}$ because the incentive constraint is assumed to be binding for all $\rho$ (Assumption 3 ).

Combining these observations, it is immediate that for $\rho$ close to one $W_{\text {sep }}(\rho)>$ $W_{\text {pool }}(\rho)$ since the dynamic benefits are approximately zero and $\Delta \omega(\rho)<0$. In the proof, we show that the static benefits of pooling $\Delta \omega(\rho)$ are increasing in $\rho$ for low levels of reputation. Intuitively, in the pooling regime the rule designer is inducing the optimizing type to follow a more stringent policy than the static best response, $\pi_{1, \text { ico }}(\rho)<\pi^{*}\left(x_{0}(\rho)\right)$ at the cost of forcing the commitment type to follow a more lenient policy, $\pi_{1, \text { ico }}(\rho)>\underline{\pi}$. When reputation is low enough, this makes the expected policy more stringent in the pooling regime than in the separating regime, because in the latter, the private agents expect the recommended policy to be followed with a low probability. Thus, pooling has both static and dynamic benefits and is therefore preferable.

For the Barro-Gordon example we can provide a tighter characterization of the optimal policy and show that it has a cutoff property. The proof for this is in the appendix. (For the bailout example we verify that this is the case numerically.) The optimal dynamic rule in this case is plotted in Figure 3.

Let's now consider what Proposition 1 implies for our two examples. In the bailout example, the optimal static rule is a strict no-bailout policy. However, in the dynamic 
Figure 2: Dynamic and static benefits of pooling

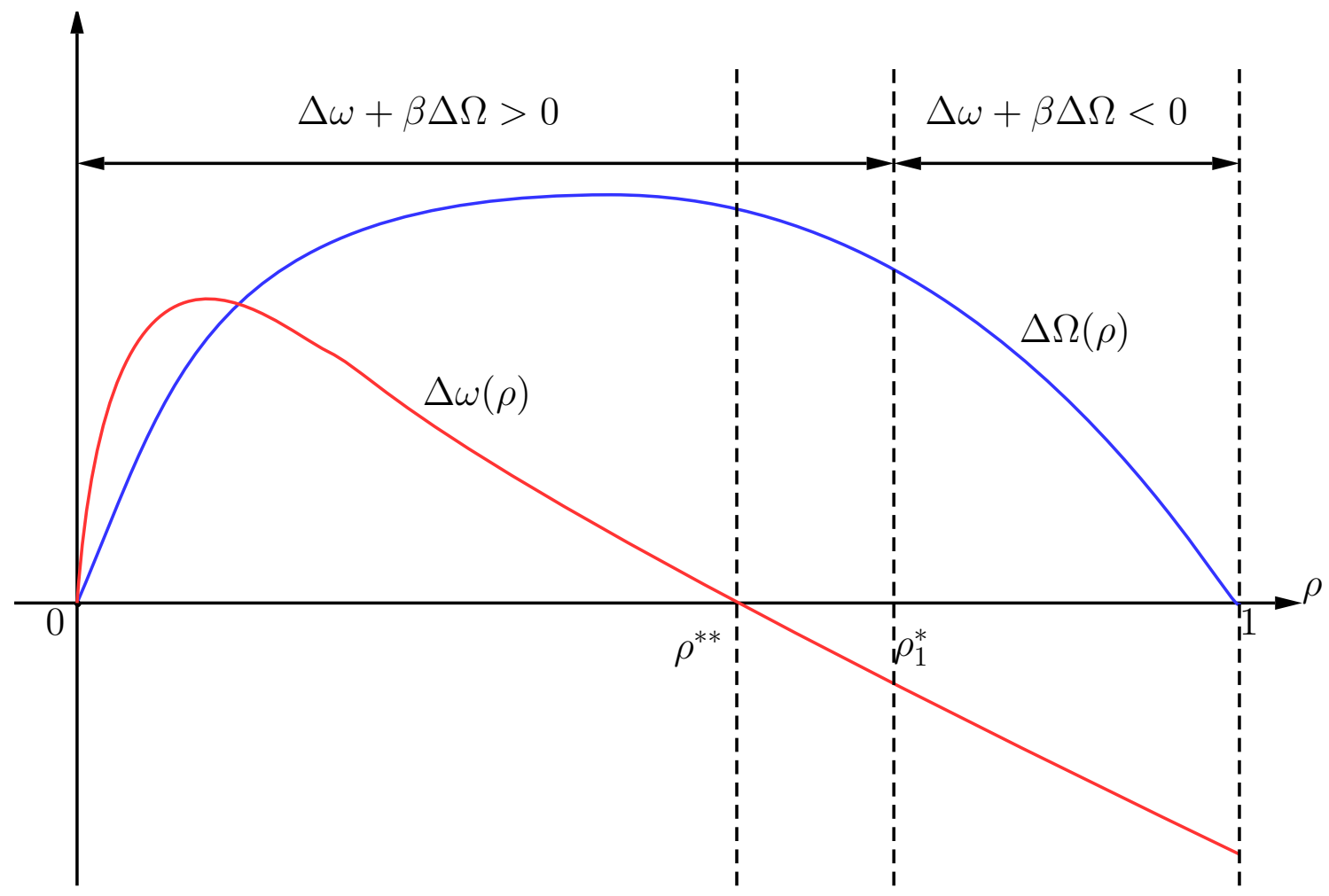

model, on-path bailouts are necessary to achieve good outcomes when reputation is low. In particular, counter to conventional wisdom, bailouts along the equilibrium path are necessary in order to impose future discipline on financial institutions. This is precisely because allowing for bailouts makes it easier for the optimizing type to follow the designer's recommendation and thus helps to preserve uncertainty going forward. This is beneficial because uncertainty about the policy maker's type prevents bankers from taking on excessive risk by exerting little effort. Similarly, in the Barro-Gordon model, having looser inflation targets is beneficial when reputation is low.

\subsection{Limit of finite horizon}

We now show that the insights from the two-period model extend to any horizon. In particular, we analyze the limit of the finite horizon economy and show that an analog of Proposition 1 holds.

Let $K$ be the horizon of the economy. For a fixed $K$, let $\left\{\pi_{k}^{K}(\rho)\right\}_{k=0}^{K}$ be the optimal rules set by the rules designer at each horizon. In the previous section, we characterized the case for $\mathrm{K}=1$. We will use the following property: 
Figure 3: Optimal dynamic rule

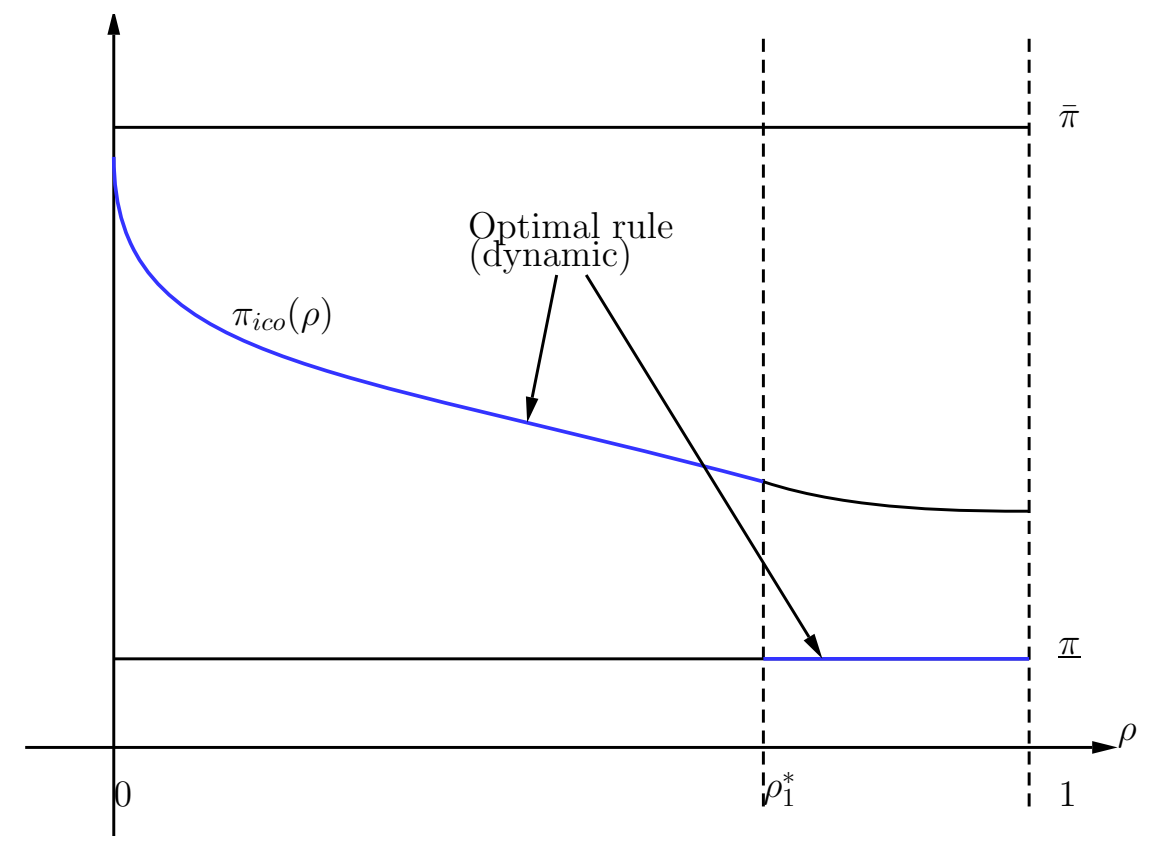

Assumption 4. The gains of best responding are decreasing in $x$, in that

$$
\mathrm{G}(\mathrm{x}) \equiv w\left(x, \pi^{*}(\mathrm{x})\right)-w\left(x, \phi^{-1}(\mathrm{x})\right)
$$

is monotone decreasing in $\mathrm{x}$.

This property is satisfied in our two examples. In the appendix we provide an additional sufficient condition on the general environment which implies this property.

To set up our next proposition, we define the following objects. First, let $\left(x_{\mathrm{CK}}, \pi_{\mathrm{CK}}\right)$ be the private action and the policy that emerge in the best sustainable equilibrium for the infinite horizon version of the model where $\rho=0$. That is, $x_{C K}$ solves

$$
\frac{w\left(x_{C K}, \phi^{-1}\left(x_{C K}\right)\right)}{1-\beta_{\mathrm{o}}}=w\left(x_{C K}, \pi^{*}\left(x_{C K}\right)\right)+\frac{\beta_{\mathrm{o}}}{1-\beta_{\mathrm{o}}} W_{0}(0),
$$

where $W_{0}(0)=V_{0}(0)$ is the value of the worst equilibrium (the repetition of the static Nash for $\rho=0)$ and $\pi_{C K}=\phi^{-1}\left(x_{C K}\right)$. Note that because of Assumption 3, $x_{C K}$ is higher (lower) than the Ramsey outcome when $w_{x}<0\left(w_{x}>0\right)$.

Second, define the cutoff $\rho^{*}$ as the (unique) solution to

$$
\frac{w\left(x_{C K}, \phi^{-1}\left(x_{C K}\right)\right)}{1-\beta}=W_{0}\left(\rho^{*}\right)+\frac{\beta}{1-\beta}\left[\rho^{*} W_{0}(1)+\left(1-\rho^{*}\right) W_{0}(0)\right] .
$$

Recall that $\rho_{1}^{*}$ is the cutoff that separates the region where it is optimal to pool from the 
region where it is optimal to separate in the twice repeated Barro-Gordon economy. For the next result we restrict ourselves to environments in which such a cutoff exists.

The next proposition shows that the limit of the finite horizon economy has the following property: there are two cutoffs and it is optimal to pool for priors below one cutoff and separate for priors above the other cutoff.

Proposition 2. Under Assumptions 1-4, as the horizon $\mathrm{k} \rightarrow \infty$ we have that:

1. For $\rho=0, W_{k}(0)=W_{0}(0) /(1-\beta)$ and $V_{k}(0)=V_{0}(0) /\left(1-\beta_{0}\right)$.

2. For $\rho \in\left(0, \rho^{*}\right]$, there is pooling for all $\mathrm{k}$ and $\pi_{\mathrm{k}} \rightarrow \pi_{\mathrm{CK}}$.

3. For $\rho \in\left(\rho_{1}^{*}, 1\right]$, there is separation for all $\mathrm{k}$ and $\pi_{\mathrm{k}}=\underline{\pi}$ for all $\mathrm{k}$.

4. For $\rho \in\left(\rho^{*}, \rho_{1}^{*}\right)$ there is no convergence. In particular, the optimal rules display a cyclical pattern: it is optimal to pool for $\mathrm{M}(\rho)$ consecutive periods and then to separate for one period and so on.

Qualitatively, the optimal rule is the same as in the two-period model. For high values of $\rho$, above the cutoff $\rho_{1}^{*}$, it is optimal to separate because pooling is associated with static losses that are not compensated by the dynamic gains. For low levels of reputation, it is optimal to choose rules that do not reveal the type of the policy maker. Note that the optimal policy in the pooling regime does not depend on the prior $\rho$ in the limit. This is because if it is optimal to pool today it is also optimal to pool in all subsequent periods. In this case, the type of the policy maker will never be revealed and so $\rho$ does not affect the value on the equilibrium path. The initial prior also does not affect the value of the deviation on the right side of (10). This is because upon deviation the prior jumps to zero independently of the initial value. Thus the value of pooling in $\left(0, \rho^{*}\right]$ is independent of $\rho$, as shown in Figure 4. Moreover, the policy converges to its value in the best sustainable equilibrium when it is known that the policy maker is the optimizing type.

Notice that there is a discontinuity at $\rho=0$. This is because when $\rho=0$ and the horizon is finite it is not possible to incentivize the optimizing type to choose any policy other than its static best response.

For intermediate values, $\rho \in\left(\rho^{*}, \rho_{1}^{*}\right)$, the equilibrium strategies do not converge as the horizon goes to infinity. This is because there is strategic substitutability between rule designers in different periods. Pooling by the rule designer in period $t+1$ reduces the incentives of the period $t$ rule designer to pool. In fact, the prospect of pooling in $t+1$ tightens the incentive compatibility for the optimizing type in period $t$. The best way to provide incentive to the optimizing type is to promise separation next period. We will explain the intuition behind this observation in Section 3.4 when discussing optimal rule design when the rule designer can commit. 
Comparison with best sustainable equilibrium We now compare the limit of the finite horizon to the best sustainable equilibrium (in the infinite horizon economy).

Proposition 3. Under Assumption 3, the best sustainable equilibrium from the rule designer's perspective is such that it is always optimal to separate for all $\rho>0$. The value to the rule designer is larger than the limit of the finite horizon for $\rho \in(0,1)$.

The value of the best equilibrium is plotted in Figure 4 and denoted by $\bar{W}(\rho)$. In the best sustainable equilibrium, it is always optimal to separate even when uncertainty is beneficial in the finite horizon economy. This is because trigger strategies can substitute for reputation and it is statically beneficial to use the commitment power of the commitment type. In fact, the value of the pooling regime equals the value of the best equilibrium when the rule designer knows that it is facing the optimizing type for sure, $W_{\text {pool }}=\bar{W}(0)$. This is because when $\rho=0$, it is possible to support $\pi_{\mathrm{CK}}$ with trigger strategies to the worst equilibrium, $\underline{W}(0)$, which equals $W_{0}(0) /(1-\beta)$. Instead, in the limit of the finite horizon economy, once the private agents learn that the policy maker is the commitment type, the only outcome that can be supported is the repetition of the static economy with $\rho=0$ with value $W_{0} /(1-\beta)=\underline{W}(0)$.

\section{Figure 4: Equilibrium values}

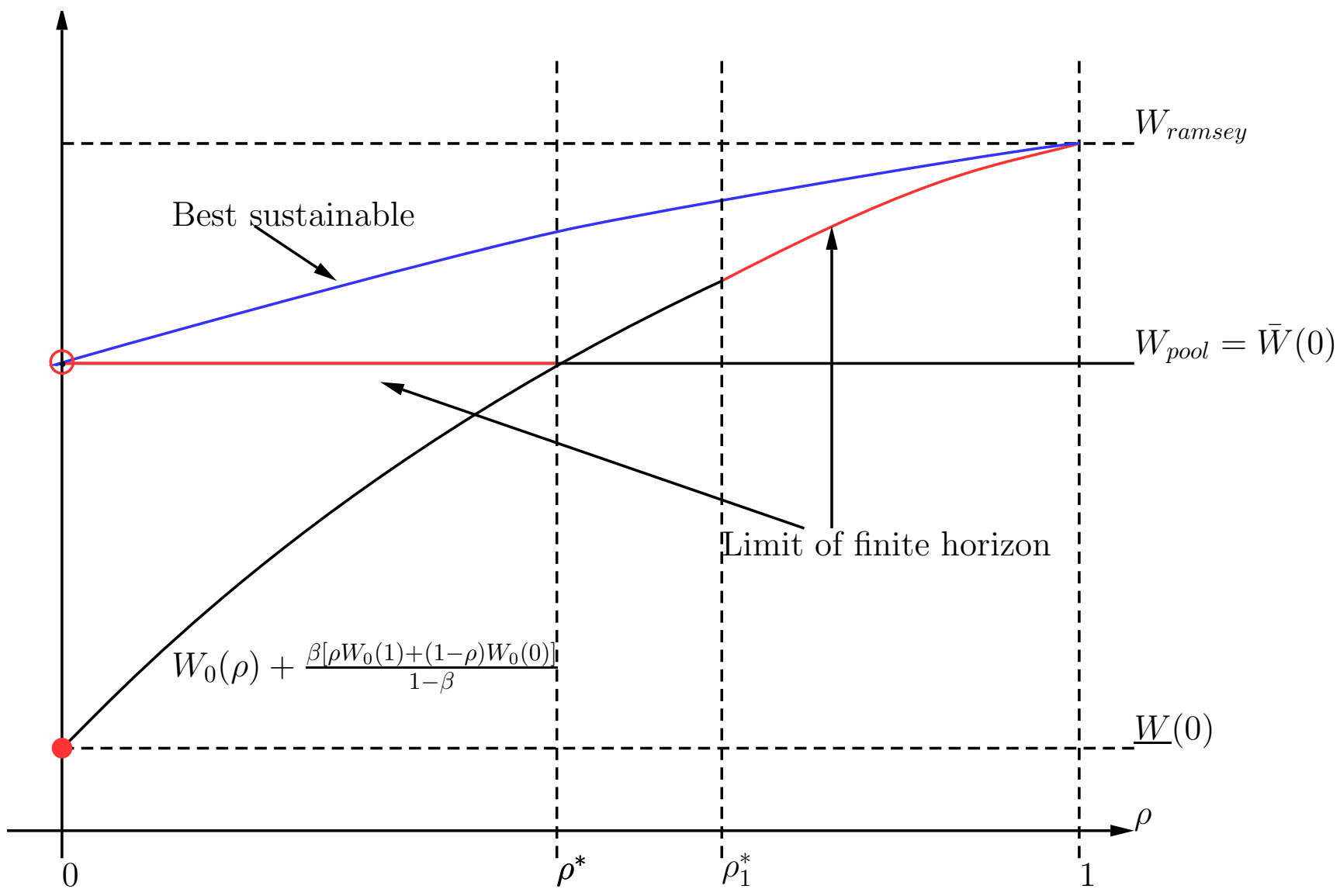




\subsection{Optimal rules when the rule designer can commit}

In our baseline model, we assumed that the rule designer chooses the optimal rule in each period. We now study the problem for the rule designer that can choose rules for all subsequent periods in period zero and commit to them. For an intermediate range of priors, the solution to this problem differs from the case in which rules are chosen sequentially: the rule designer itself suffers from a time-inconsistency problem. This is because future rules can be used to incentivize the policy maker in the current period, thereby relaxing the incentive compatibility constraint. We illustrate this point in the simplest possible way by considering a thrice repeated economy. ${ }^{9}$

The key insight is that the optimizing type's incentive constraint in period $t$ is tighter if there is pooling in period $t+1$ as compared with the case in which there is separation in $t+1$ for sufficiently high levels of reputation. Thus the period $t$ rule designer wants to have more stringent rules in period $t+1$ to induce separation. To understand this point, consider the value of the optimizing type along the equilibrium path in the first two periods, when rules are chosen sequentially. These values are illustrated in Figure 5. Consider the value in period $1, V_{1}(\rho)$. This value is discontinuous at the cutoff $\rho_{1}^{*}$. This is because at $\rho_{1}^{*}$, the period-one rule designer is indifferent between pooling and separating. Since separation has dynamic losses, it must have static gains. A necessary condition for separation to be statically beneficial over pooling is that the private action under pooling, $x_{i c o, 1}(\rho)$, is worse than the one under separation, i.e., $x_{i c o, 1}(\rho)>x_{0}(\rho)$ when $w_{x}<0$. This in turn implies that

$$
\begin{aligned}
\lim _{\rho \uparrow \rho_{1}^{*}} \bigvee_{1}(\rho) & =w\left(x_{i c o, 1}\left(\rho_{1}^{*}\right), \phi^{-1}\left(x_{i c o, 1}\left(\rho_{1}^{*}\right)\right)\right)+\beta V_{0}(\rho) \\
& =w\left(x_{i c o, 1}\left(\rho_{1}^{*}\right), \pi^{*}\left(x_{i c o, 1}\left(\rho_{1}^{*}\right)\right)\right)+\beta \bigvee_{0}(0) \\
& <w\left(x_{0}\left(\rho_{1}^{*}\right), \pi^{*}\left(x_{0}\left(\rho_{1}^{*}\right)\right)+\beta \bigvee_{0}(0)=\lim _{\rho \nmid \rho_{1}^{*}} V_{1}(\rho),\right.
\end{aligned}
$$

where the second equality follows from (6) and the strict inequality follows from $x_{i c o, 1}(\rho)>$ $x_{0}(\rho)$. The idea is that the optimizing type's value is high when the private action is low and it is allowed to best respond.

Consider now the incentive compatibility constraint in the first period. This can be written as

$$
w(x, \pi)-w\left(x, \pi^{*}(x)\right) \geqslant \beta_{0}\left[V_{1}(\rho)-V_{1}(0)\right]
$$

For $\rho$ slightly smaller than $\rho_{1}^{*}$, from (11) we have that $V_{0}(\rho)-V_{0}(0)>V_{1}(\rho)-V_{1}(0)$, as illustrated in Figure 5. Thus the incentive constraint is tighter in period zero than in

\footnotetext{
${ }^{9}$ Note that in the two-period economy there is no difference between the date zero and sequentially optimal rule since the only outcome that is feasible in last period is separation.
} 
Figure 5: Dynamic incentives for the optimizing type, $V_{k}(\rho)-V_{k}(0)$ for $k=0,1$

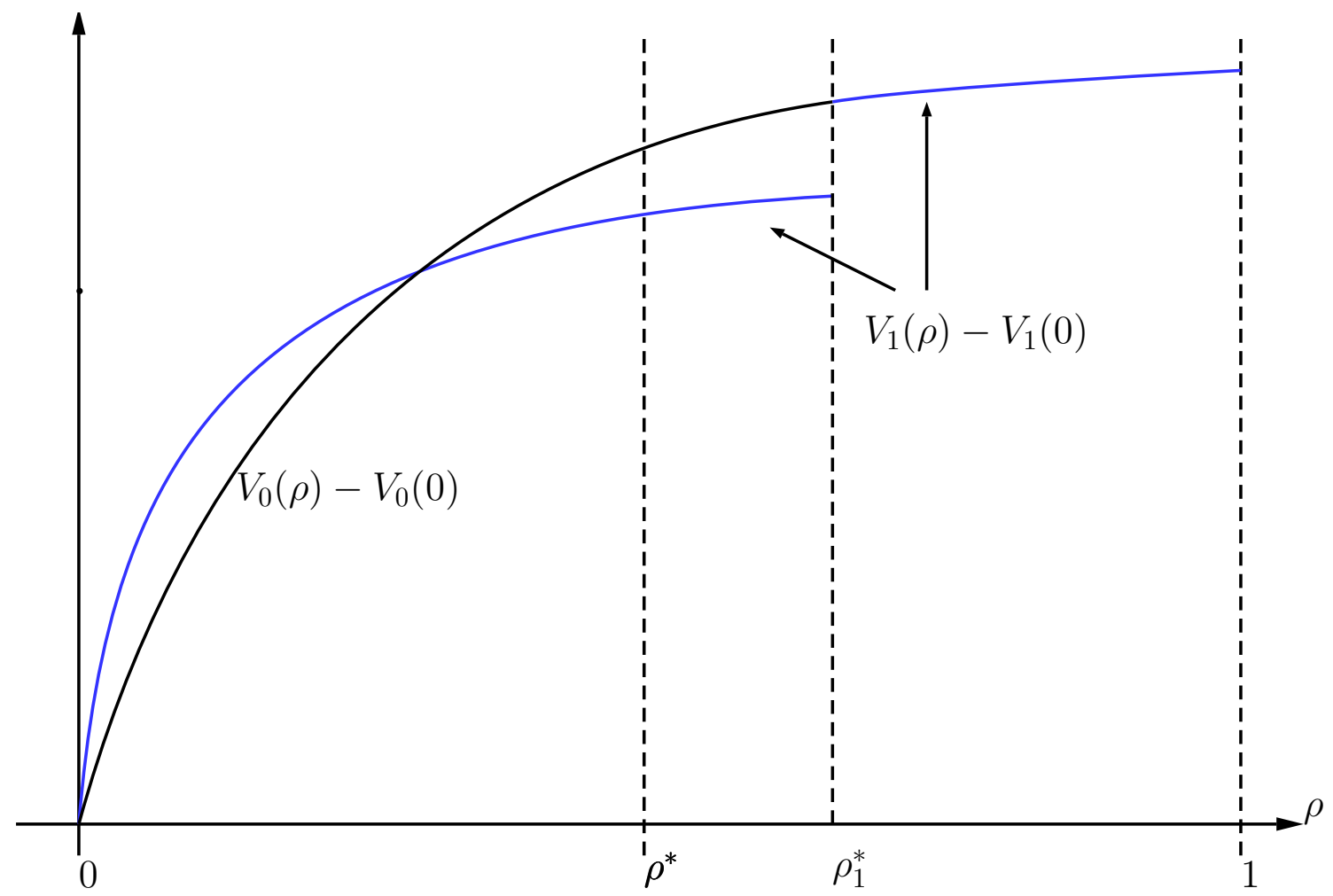

period one. Therefore, the static value of pooling is lower and the region in which it is optimal to pool shrinks. Then, there are priors $\rho$ such that it is optimal to pool in period one but optimal to separate in period zero, when rules are chosen sequentially. For those levels of reputation, the rule designer in period zero would like to force the rule designer in period one to adopt a stringent rule that induces separation next period and relax its incentive compatibility constraint. We have the following proposition:

Proposition 4. There exists an interval $\left[\rho_{\text {commit }}^{*}, \rho_{1}^{*}\right]$ where the optimal rules with and without commitment do not coincide. In particular, without commitment, it is optimal to separate in period zero. With commitment, it is optimal to pool in period zero and commit to separation in period one. This is achieved by committing to the most stringent rule, $\underline{\pi}$, in period one.

\subsection{Sticky rules}

So far we have allowed the rule designer to choose a new rule in each period as a function of the current reputation of the policy maker. In practice, opportunities for revising and introducing new rules arise infrequently. We now modify our framework to allow for this feature and show that our main conclusions are unchanged. In particular, the characterization in Proposition 2 continues to hold.

We consider the case in which rules are "sticky" in that they can only be changed in a 
given period with probability $\alpha$. The analyses in the baseline modelconsidered the case in which $\alpha=1$. We now assume that $\alpha<1$. Let $\tilde{W}_{t+1}\left(\rho^{\prime}, \pi_{\mathfrak{c}}\right)$ be the rule designer's value next period if it cannot set a new rule and must use $\pi_{\mathrm{c}}$ and $\tilde{V}_{\mathrm{t}+1}\left(\rho_{\mathrm{o}}^{\prime}, \pi_{\mathrm{c}}\right)$ be the analogous value for the optimizing type. Fixing the horizon $K$, the problem for a rule designer that has the opportunity to set new rules in period $t<K$ can be written as

$$
\begin{aligned}
W_{\mathrm{t}}(\rho)=\max _{x, \pi_{\mathrm{c}}, \pi_{\mathrm{o}}} \rho\left[w\left(x, \pi_{\mathrm{c}}\right)+\beta \alpha W_{\mathrm{t}+1}\left(\rho_{\mathrm{c}}^{\prime}\right)+\beta(1-\alpha) \tilde{W}_{\mathrm{t}+1}\left(\rho_{\mathrm{c}}^{\prime}, \pi_{\mathrm{c}}\right)\right] \\
+(1-\rho)\left[w\left(x, \pi_{\mathrm{o}}\right)+\beta \alpha W_{\mathrm{t}+1}\left(\rho_{\mathrm{o}}^{\prime}\right)+\beta(1-\alpha) \tilde{W}_{\mathrm{t}+1}\left(\rho_{\mathrm{o}}^{\prime}, \pi_{\mathrm{c}}\right)\right]
\end{aligned}
$$

subject to the implementability condition (4), the evolution of the prior (7), and the incentive compatibility constraint

$$
w\left(x, \pi_{\mathrm{o}}\right)+\beta \alpha V_{\mathrm{t}+1}\left(\rho_{\mathrm{o}}^{\prime}\right)+\beta(1-\alpha) \tilde{V}_{\mathrm{t}+1}\left(\rho_{\mathrm{o}}^{\prime}, \pi_{\mathrm{c}}\right) \geqslant w\left(x, \pi_{\mathrm{o}}\right)+\beta \mathrm{V}_{\mathrm{t}+1}(0)
$$

Under our assumptions, if the rule designer wants to separate, its value is the same as the one in the previous section since the optimal rule under separation is $\underline{\pi}$ for all $\rho$ and t. However, the introduction of sticky policies affects the rule designer's value when it chooses to pool. In particular, choosing $\pi_{\mathrm{c}}$ equal to the lowest value consistent with the optimizing type's incentive compatibility constraint induces the optimizing type to pool in the current period, but it may induce separation in future periods if the rules cannot be adjusted. Thus the rule designer may want to choose an even more lenient policy, which implies that (12) is slack, to ensure pooling next period in the event that the rule cannot be adjusted. However, this trade-off vanishes in the limit for $\rho \in\left[0, \rho^{*}\right]$ as the horizon goes to infinity since

$$
\lim _{K \rightarrow \infty} \pi_{i c o, t}^{K}(\rho)=\lim _{K \rightarrow \infty} \pi_{i c o, t+1}^{K}(\rho)=\pi_{C K}
$$

where $\pi_{\mathrm{CK}}$ is defined in (10). Thus the minimal rule that ensures pooling is constant over time. This observation implies that Proposition 2 holds with sticky policies $(\alpha<1)$.

Note that the ability to change the rule only with some probability does not help the rule designer to solve the underlying time inconsistency problem. This is because the optimal sequence of rules from a time zero perspective are time and state varying. In fact, as we showed in the previous section, the date zero rule designer would choose different rules for period zero and one. The stickiness here limits only the ability to change rules over time. 


\section{Transparency of rules}

We now study the implications of our theory for the optimal degree of transparency of the rule. Should the rule be designed so that a deviation by the policy maker is easily detectable? In other words, we ask if perfect monitoring is always desirable. Conventional wisdom suggests that for a typical repeated policy game with no reputational considerations, perfect monitoring is always desirable. In contrast, we show that with reputational considerations, perfect monitoring is desirable only for low levels of reputation, while imperfect monitoring is desirable for high levels of reputation.

\subsection{Optimal degree of monitoring}

We first consider the case in which the rule designer can control the degree to which the private agents and future rule designers can monitor the policies chosen by the policy maker. In particular, suppose the private agents cannot directly observe the policy $\pi$, but they can only observe a signal $\tilde{\pi}=\pi+\varepsilon$, where $\varepsilon \sim N\left(0, \sigma_{\varepsilon}^{2}\right)$. The rule designer can choose the standard deviation of the noise, $\sigma_{\varepsilon}$, as part of the optimal design of the rule. We interpret a choice of large noise as standing in for complicated rules whose deviations are hard to detect for the private agents. We say that a rule is transparent if $\sigma_{\varepsilon}=0$ and opaque if $\sigma_{\varepsilon}>0$.

For a given $\sigma_{\varepsilon}$, the law of motion for beliefs is

$$
\rho^{\prime}(\tilde{\pi}, \rho)=\frac{\rho \operatorname{Pr}\left(\tilde{\pi} \mid \pi_{c}\right)}{\rho \operatorname{Pr}\left(\tilde{\pi} \mid \pi_{c}\right)+(1-\rho) \operatorname{Pr}\left(\tilde{\pi} \mid \pi_{\mathrm{o}}\right)}=\frac{\rho g\left(\tilde{\pi}-\pi_{\mathrm{c}}\right)}{\rho g\left(\tilde{\pi}-\pi_{\mathrm{c}}\right)+(1-\rho) g\left(\tilde{\pi}-\pi_{\mathrm{o}}\right)^{\prime}},
$$

where $g$ is the PDF of a Normal distribution with mean zero and variance $\sigma_{\varepsilon}^{2}$. We can then write the rule designer's problem for the twice repeated economy as

$$
\begin{aligned}
& \max _{x, \pi_{\mathrm{c}}, \pi_{\mathrm{o}}, \sigma_{\varepsilon}} \rho {\left[\mathcal{W}\left(x, \pi_{\mathrm{c}}\right)+\beta \int W_{0}\left(\rho^{\prime}\left(\pi_{\mathrm{c}}+\varepsilon, \rho\right)\right) g(\varepsilon) \mathrm{d} \varepsilon\right] } \\
&+(1-\rho)\left[\mathcal{W}\left(x, \pi_{\mathrm{o}}\right)+\beta \int W_{0}\left(\rho^{\prime}\left(\pi_{\mathrm{o}}+\varepsilon, \rho\right)\right) g(\varepsilon) \mathrm{d} \varepsilon\right]
\end{aligned}
$$

subject to the implementability condition, $x=\rho \pi_{c}+(1-\rho) \pi_{0}$, the incentive compatibility constraint for the optimizing type,

$w\left(x, \pi_{0}\right)+\beta_{o} \int V_{0}\left(\rho^{\prime}\left(\pi_{\mathrm{o}}+\varepsilon, \rho\right)\right) g(\varepsilon) d \varepsilon \geqslant w(x, \pi)+\beta_{\mathrm{o}} \int \mathrm{V}_{0}\left(\rho^{\prime}(\pi+\varepsilon, \rho)\right) g(\varepsilon) d \varepsilon \quad \forall \pi$,

and the law of motion for beliefs (13). Note that the values in the final period, $W_{0}$ and $V_{0}$, are the static values and are not affected by $\sigma_{\varepsilon}$. 
The next proposition establishes that for low levels of reputation it is optimal to have perfectly transparent rules $\left(\sigma_{\varepsilon}=0\right)$, while for higher values of reputation it is optimal to have opaque rules:

Proposition 5. Under Assumptions 1-4:

1. For $\rho$ close to zero there is pooling and signals are perfectly informative, $\sigma_{\varepsilon}=0$.

2. For $\rho$ close to one there is separation and signals are not perfectly informative, $\sigma_{\varepsilon}>0$.

Consider first low levels of reputation. From Proposition 1, we know that if signals are perfectly informative, it is optimal to be in the pooling regime so $\pi_{\mathrm{o}}=\pi_{\mathrm{c}}$. Conditional on pooling, it is preferable to choose $\sigma_{\varepsilon}=0$ to relax the incentive constraint (15). In fact, without noise, (15) reduces to

$$
w\left(x, \pi_{0}\right)+\beta_{0} V_{0}(\rho) \geqslant w(x, \pi)+\beta_{0} V_{0}(0) \quad \forall \pi
$$

and so the spread in continuation values $\left[V_{0}(\rho)-V_{0}(0)\right]$ provides the maximal incentives to the optimizing type. To see this, first note that for any $\sigma_{\varepsilon}>0$

$$
\int \mathrm{V}_{0}\left(\rho^{\prime}(\pi+\varepsilon, \rho)\right) g(\varepsilon) d \varepsilon>V_{0}(0)
$$

so the right side of (15) is the lowest at $\sigma_{\varepsilon}=0$. Second, by concavity of $V_{0}$ we have that

$$
V_{0}(\rho)>\int V_{0}\left(\rho^{\prime}\left(\pi_{o}+\varepsilon, \rho\right)\right) g(\varepsilon) d \varepsilon
$$

since $\rho=\int \rho^{\prime}\left(\pi_{\mathrm{o}}+\varepsilon, \rho\right) \mathrm{g}(\varepsilon) \mathrm{d} \varepsilon$, so the left side of (15) is the highest at $\sigma_{\varepsilon}=0$. Thus, since we know that for low levels of reputation pooling is preferable to separating we have that the optimal rule has pooling and it is perfectly transparent.

Consider now high levels of reputation. Suppose by way of contradiction that it is optimal to be in the separating regime $\left(\pi_{\mathrm{o}} \neq \pi_{\mathrm{c}}\right)$ with perfectly informative signals, $\sigma_{\mathcal{E}}=$ 0 . Since types are perfectly revealed at the end of the first period we have that $\rho^{\prime} \in\{0,1\}$ and the only incentive-compatible policy for the optimizing type is $\pi_{\mathrm{o}}=\pi^{*}(\mathrm{x})$. Note that we can support the same policies by choosing $\sigma_{\varepsilon}=\infty$. This alternative rule has the same static payoff but prevents learning about the regulator's type and therefore $\rho^{\prime}=\rho$ because the signal $\tilde{\pi}$ is totally uninformative. This increases the expected continuation value because uncertainty is beneficial, $W(\rho)>\rho W(1)+(1-\rho) W(0)$. Thus, the rule designer's payoff is strictly higher and therefore it cannot be that $\sigma_{\varepsilon}=0$. In principle, it may be optimal to choose an intermediate value for the noise $\sigma_{\varepsilon}$ to induce the optimizing type to do something better than the static best response. 


\subsection{Optimal tenure}

The results in Proposition 5 are also informative about the optimal tenure of the policy maker. In fact, an alternative instrument for the rule designer to separate the static policy choice from the evolution of the reputation of the policy maker in subsequent periods is to terminate the current policy maker's tenure after one period. This is equivalent to choosing a perfectly opaque rule with $\sigma_{\varepsilon}=\infty$. Thus early termination (one-period tenure) is optimal when the reputation of a new policy maker is sufficiently high.

Consider the twice repeated environment. To obtain a tighter characterization we prove our result for the Barro-Gordon model. Suppose that in the first period the rule designer can choose a regulation $\pi_{c}$ and whether to terminate the policy maker's tenure after one period. The prior that a new policy maker is the commitment type is $\rho$ and is constant in both periods. We assume that the termination choice cannot be made contingent on the outcome at the end of the period. It is clear that the rule designer's problem is the same as the one in (14) with the additional restriction that $\sigma_{\varepsilon} \in\{0, \infty\}$.

Proposition 6. In the Barro-Gordon model, there exists $\rho^{* *}<\rho_{1}^{*}$ such that:

1. For $\rho \leqslant \rho^{* *}$ it is optimal to pool and not terminate the policy maker's tenure after one period.

2. For $\rho \geqslant \rho^{* *}$ it is optimal to separate and terminate the policy maker's tenure after one period.

Consider first the case in which pooling has static benefits, $\Delta \omega(\rho) \geqslant 0$. As shown in Figure 2, this is true for $\rho \in\left[0, \rho^{* *}\right]$, where $\rho^{* *}$ is defined as $\Delta \omega\left(\rho^{* *}\right)=0$. In this case, the rule designer does not want to terminate the policy maker's tenure, as it would tighten the incentive compatibility constraint without changing the continuation value. Thus in this region it is optimal to not terminate the policy maker's tenure.

Consider next the case in which there are static losses of pooling in that $\Delta \omega(\rho)<0$. This is true for levels of reputation above the cutoff $\rho^{* *}$. For these levels of reputation, if the rule designer keeps the same policy maker in office, the rule designer must trade off the static losses of pooling against their dynamic benefits. However, when the rule designer terminates the policy maker's tenure after one period, it can achieve both the static benefits associated with separation and the dynamic benefits associated with pooling. This is because replacing the policy maker after one period prevents learning and does not require the commitment type to implement a lenient policy in order to do so.

\subsection{Stochastic rules}

An alternative way of introducing opacity in rules is to allow the rule designer to choose stochastic rules even though fundamentals are deterministic. The rule designer can now choose a rule that consists of a set of policies, $\Sigma_{c}$, and a probability distribution over 
these policies, $\sigma_{c}$. We can interpret this as introducing clauses that allow policies to be conditioned on irrelevant details. The commitment type will then draw a policy from this distribution. The optimizing type can also randomize across policies. We will denote its strategy as $\sigma_{0}$.

The rule designer's problem is then

$$
\max _{\chi, \sigma_{0}, \sigma_{c}} \int\left[w(x, \pi)+\beta W_{0}\left(\rho^{\prime}(\pi, \rho)\right)\right]\left[\rho \sigma_{c}(\pi)+(1-\rho) \sigma_{o}(\pi)\right] d \pi
$$

subject to $\sigma_{\mathrm{c}}, \sigma_{\mathrm{o}} \in \Delta([\underline{\pi}, \bar{\pi}])$, the implementability condition,

$$
\chi=\phi\left(\int \pi\left[\rho \sigma_{c}(\pi)+(1-\rho) \sigma_{\mathrm{o}}(\pi)\right] \mathrm{d} \pi\right),
$$

the incentive compatibility constraint for the optimizing type, $\forall \pi \in S u p p \sigma_{0}, \forall \tilde{\pi} \in \operatorname{Supp} \sigma_{0} \cup$ Supp $\sigma_{\mathrm{c}} \cup\left\{\pi^{*}(\mathrm{x})\right\}$

$$
w(x, \pi)+\beta V_{0}\left(\rho^{\prime}(\pi, \rho)\right) \geqslant w(x, \tilde{\pi})+\beta V_{0}\left(\rho^{\prime}(\tilde{\pi}, \rho)\right),
$$

and the evolution of beliefs,

$$
\rho^{\prime}(\pi, \rho)=\frac{\rho \sigma_{\mathcal{c}}(\pi)}{\rho \sigma_{\mathcal{c}}(\pi)+(1-\rho) \sigma_{\mathrm{o}}(\pi)}
$$

We say that a rule is stochastic if the support of $\sigma_{\mathcal{c}}$ contains more than one element, while a rule is deterministic if the support of $\sigma_{c}$ is a singleton. Similar to Proposition 5, we show that if the policy maker's reputation is high enough then recommending stochastic rules is optimal, while if its reputation is sufficiently close to zero then it is optimal to have deterministic rules that provide strong incentives for the optimizing type.

Proposition 7. Suppose Assumptions 1-4 hold:

1. For $\rho$ close to one it is optimal to have stochastic rules.

2. For $\rho$ close to zero a deterministic rule is optimal and, in particular, $\pi_{\mathrm{c}}=\pi_{\mathrm{ico}}(\rho)$ with probability one.

Consider first the case in which the reputation is close to one. The optimality of stochastic rules follows from the properties of Bayes' rule and continuation values being increasing in the prior and does not rely on uncertainty being beneficial. To establish the result, suppose by way of contradiction that it is optimal to choose a rule that recommends policy $\underline{\pi}$ with probability one. This is the best deterministic rule, as shown in Proposition 1. Consider a perturbation in which the rule puts a small but positive probability, $\varepsilon$, on the static best response. When $\rho$ is close to one, on observing the static best 
response, the agents attribute this to the perturbation of the commitment type rather than the optimizing type. Consequently, the posterior that the policy maker is the commitment type rises sharply, which increases the expected continuation value of the perturbation and more than compensates the static losses. ${ }^{10}$

The case with reputation close to zero instead relies on uncertainty being beneficial. The argument here mirrors the one provided to show that randomization by the optimizing type is not optimal. The idea here is that randomization tightens the optimizing type's incentive constraint, which results in a more lenient expected policy. This in turn lowers the static payoff in addition to the dynamic losses that arise because uncertainty is beneficial.

The message of this section is that when reputation is low, rules should be transparent and easily interpretable so that deviations are easily detectable. This is because providing incentives to the optimizing type is critical, as in Atkeson et al. (2007). In contrast, when reputation is high, rules should be opaque and hard to interpret. This is because the benefits of maintaining uncertainty about the policy maker's type outweigh the costs associated with looser incentives to the optimizing type. This can account for why countries with low credibility adopt policies like fixed exchange rates or crawling pegs, while countries with high credibility are more likely to have discretionary exchange rate policies.

\section{Signaling game and payoff types}

In this section, we contrast our characterization of the optimal rule in Section 3 with two alternatives. First, we consider a signaling game in which the rule is chosen by the policy maker (which knows its type) instead of the rule designer, which is uncertain about the type of the policy maker. Second, we consider a model where the two types of policy makers differ in their preferences. In particular, policy makers can differ in their temptation to deviate ex-post because certain policy makers can better resist pressure from interest groups ex-post or have different preferences over outcomes than the social welfare function, as in the seminal Rogoff (1985) paper. We show that in both cases the equilibrium outcome has separation for all levels of reputation (under a reasonable refinement), in contrast with our main result that it is optimal to pool for low levels of reputation.

\footnotetext{
${ }^{10}$ Note that forcing the commitment type to randomize reduces the variance of the posterior. In fact, under the deterministic rule with separation, the posterior is one with probability $\rho$ and zero with probability $1-\rho$. Under our perturbation, the posterior is one with probability $\rho(1-\varepsilon)$ and $\rho \varepsilon /[\rho \varepsilon+(1-\rho)]$ with probability $\rho \varepsilon+(1-\rho)$.
} 


\subsection{Comparison to a signaling game}

We now consider a signaling game in which the rule is chosen by the policy maker (which knows its type) instead of the rule designer, which is uncertain about the type of the policy maker. If the rules are chosen by the policy makers, the commitment type (if sufficiently patient) will choose a rule that induces separation for all levels of reputation. In particular, it will prefer to separate for low levels of reputation even though the rule designer strictly prefers to pool. This result mirrors the one in Dovis and Kirpalani (2019a).

Proposition 8. Under Assumptions 1 and 3, the outcome of the signaling game is such that the commitment and optimizing types follow different policies if either i) $\rho$ is sufficiently high or ii) $\rho$ is sufficiently small and $\beta_{o}=\beta_{c} \in(\underline{\beta}, \bar{\beta})$. Thus, in both cases there is separation after one period.

The main idea here is that there are no dynamic gains for the commitment type of preserving uncertainty. The continuation value for the commitment type is always larger in a separating equilibrium as compared with pooling, as it can achieve the Ramsey outcome since the private agents know that they are facing the commitment type. However, there may still be static benefits of pooling when reputation is sufficiently low, as we saw in Section 3.2. But if the discount factor is sufficiently high $(\beta>\beta)$, the dynamic benefits outweigh the static losses. Note that for this to be an equilibrium we also need the optimizing type to strictly prefer to separate, which requires the discount factor to be low enough $(\beta<\bar{\beta})$. We show that $\underline{\beta}<\bar{\beta}$, since the optimizing type has additional static benefits of separating owing to the fact that it can choose its policy after the private agents have chosen their action.

\subsection{Payoff types}

So far, we have modeled the commitment type as a policy maker that cannot deviate from the rule. An alternative to modeling the uncertainty about the policy maker's ability to follow the rule is to assume that the two types of policy makers differ in their preferences. In particular, the policy makers can differ in their temptation to deviate ex-post because certain policy makers can better resist pressure from interest groups ex-post or have different preferences over outcomes than the social welfare function, as in the seminal Rogoff (1985) paper.

We next show that with preference types and a reasonable equilibrium refinement, we have different outcomes than in our benchmark case. In particular, the equilibrium coincides with the outcome of the signaling game and there is separation for all levels of initial reputation. 
We make our point in the context of the bailout example. Recall that the social welfare function is

$$
w(x, \pi ; \psi)=-v(x)+p(x) R_{H}-\psi(1-p(x))(1-\pi)-c(\pi)
$$

where $x$ is the banker's effort given by $\phi(\mathbb{E} \pi)$ for some $\phi$ with $\phi^{\prime}<0, \phi^{\prime \prime}>0, p(x)$ is the probability that the investment succeeds, and $\psi(1-p(x))(1-\pi)$ is the default cost that can be mitigated by transfers $\pi$. The parameter $\psi$ controls the degree of time inconsistency: if $\psi=0$ then the Ramsey outcome is sustainable because there are no benefits of deviating from the optimal plan ex-post. In contrast, if $\psi$ is large then there is a much larger temptation to deviate ex-post.

Suppose now that there are two types of policy makers, each associated with a different value of $\psi$. The high cost type has $\psi=\psi_{\mathrm{H}}>0$, and the low cost type has $\psi=\psi_{\mathrm{L}}=0$. The low cost type then has no incentive to deviate ex-post and thus represents the commitment type in our baseline model. It also corresponds to the "conservative central banker" in Rogoff (1985) since if the private agents know they are facing the low cost type with probability one then the Ramsey outcome can be implemented. To keep the symmetry with the previous analyses, we assume that the social welfare function used by the rule designer to evaluate outcomes is $w\left(x, \pi ; \psi_{\mathrm{H}}\right)$.

Consider the twice-repeated problem. The characterization in the terminal period does not change relative to the case analyzed previously. Thus, the value for the rule designer is $W_{0}(\rho)$, where $\rho$ is the prior of facing the low cost type, the value for the high cost type is $V_{0}\left(\rho ; \psi_{H}\right)=V_{0}(\rho)$, and the value for the low cost type is $V_{0}(\rho ; 0)=$ $w\left(x_{0}(\rho), \pi=0\right)$.

Consider now the rule designer's problem in the first period. The difference with problem (8) is that we have to add an incentive compatibility constraint for the low cost type,

$$
w\left(x, \pi_{r} ; 0\right)+\beta_{\mathrm{o}} \mathrm{V}_{0}\left(\rho_{\mathrm{c}}^{\prime} ; 0\right) \geqslant w(x, \pi ; 0)+\beta_{\mathrm{o}} \mathrm{V}_{0}\left(\rho^{\prime}(\pi) ; 0\right) \quad \forall \pi,
$$

where $\rho^{\prime}(\pi)$ is the posterior after observing policy $\pi$ and the low cost type's discount factor is $\beta_{0}$. Since $w_{\pi}(x, \pi ; 0)=0$ for all $(x, \pi)$ then we can rewrite the constraint above as $\beta_{\mathrm{o}} \mathrm{V}_{0}\left(\rho_{\mathrm{c}}^{\prime} ; 0\right) \geqslant \beta_{\mathrm{o}} \mathrm{V}_{0}\left(\rho^{\prime}(\pi) ; 0\right)$ or, since $\mathrm{V}_{0}(\rho ; 0)$ is strictly increasing in $\rho$, as

$$
\rho_{c}^{\prime}=\rho^{\prime}\left(\pi_{r}\right) \geqslant \rho^{\prime}(\pi) \quad \forall \pi
$$

The incentive compatibility constraint for the low type, (21), is satisfied in the separation regime as $\rho_{c}^{\prime}=1$ so the rule designer can attain the same value. We now turn to analyze whether the pooling regime is feasible. The answer to this question depends on the specification of off-path beliefs. Clearly, it is possible to specify the off-path beliefs as 
follows

$$
\rho^{\prime}(\pi)=\left\{\begin{array}{ll}
\rho & \text { if } \pi=\pi_{\mathrm{r}} \\
0 & \text { if } \pi \neq \pi_{\mathrm{r}}
\end{array} .\right.
$$

This choice is consistent with Bayes' rule on-path, trivially satisfies (21), and so supports the pooling outcome described above. An unappealing feature of (22) is that implementing more stringent policies ex-post reduces the policy maker's reputation. If we restrict to specifying beliefs such that $\rho^{\prime}(\pi)$ is strictly decreasing in $\pi$ then pooling is not feasible and the separating regime is the only solution for all levels of reputation. The restriction is intuitive as it imposes that if the deviation is relatively more advantageous for the low cost type then the posterior rises after enforcement.

\section{Conclusion}

In this paper, we study the optimal design of rules in a dynamic model when there is a time inconsistency problem and uncertainty about whether the policy maker can commit to follow the rule ex-post. We show that in a large class of economies preserving uncertainty about the policy maker's type is preferable from an ex-ante perspective. Therefore, learning the type of the policy maker can be costly. When reputation is low, we show that reputational considerations imply that the optimal rule is more lenient than the one that would arise in a static environment. For example, in the context of financial regulation, on-path bailouts are necessary to discipline future risk taking by financial institutions. Moreover, opaque rules are preferable to transparent ones when reputation is high.

In our analysis we abstract from the question on the optimal degree flexibility when the policy maker has private information about the state of the economy considered by the delegation literature. See for instance Athey et al. (2005), Amador et al. (2006), and Halac and Yared (2014). In our economy with no fundamental uncertainty, if the policy maker follows the rule for sure, it is trivially optimal to leave no flexibility and by doing so implement the Ramsey outcome. An interesting avenue for future work is to study how the incentives to build reputation considered in this paper interact with the choice of how much flexibility to leave to the policy maker.

\section{References}

Amador, M. AND C. Phelan (2018): “Reputation and Sovereign Default," Tech. rep., National Bureau of Economic Research. 6 
Amador, M., I. Werning, AND G.-M. Angeletos (2006): “Commitment vs. flexibility," Econometrica, 74, 365-396. 31

Angeletos, G.-M., C. HellwiG, And A. Pavan (2006): "Signaling in a global game: Coordination and policy traps," Journal of Political economy, 114, 452-484. 7

AsRiYAN, V., D. FoARTA, V. VANASCO, ET AL. (2019): “The good, the bad and the complex: Product design with imperfect information," Tech. rep. 7

Athey, S., A. AtKeson, And P. J. KehOe (2005): “The optimal degree of discretion in monetary policy," Econometrica, 73, 1431-1475. 6, 31

AtKeson, A., V. V. ChaRi, And P. J. KehOE (2007): "On the optimal choice of a monetary policy instrument," . 5, 28

ATKESON, A. AND P. J. KEHOE (2001): “The advantage of transparent instruments of monetary policy," Tech. rep., National Bureau of Economic Research. 5

Azzimonti, M., M. BAtTAGLini, AND S. CoATE (2016): “The costs and benefits of balanced budget rules: Lessons from a political economy model of fiscal policy," Journal of Public Economics, 136, 45-61. 6

BACKUS, D. AND J. DRIFFILL (1985): "Inflation and reputation," American economic review, $75,530-538.6$

BARRO, R. J. (1986): "Reputation in a model of monetary policy with incomplete information," Journal of Monetary Economics, 17, 3-20. 3, 6

BARRO, R. J. AND D. B. GORDON (1983a): "A positive theory of monetary policy in a natural rate model," Journal of political economy, 91, 589-610. 7, 9

(1983b): "Rules, discretion and reputation in a model of monetary policy," Journal of monetary economics, 12, 101-121. 3, 6

BOND, P. AND Y. ZENG (2018): "Silence is Safest: Non-Disclosure When the Audience's Preferences are Uncertain," Available at SSRN 3124106. 7

CHARI, V. V. AND P. J. KeHOE (1990): “Sustainable plans," Journal of political economy, 98, 783-802. 6, 7

COLE, H. L., J. DOW, AND W. B. ENGLiSH (1995): “Default, settlement, and signalling: Lending resumption in a reputational model of sovereign debt," International Economic Review, 365-385. 7 
CRÉMER, J. (1995): “Arm's length relationships," The Quarterly Journal of Economics, 110, 275-295. 5

Debortoli, D. AND R. Nunes (2010): “Fiscal policy under loose commitment," Journal of Economic Theory, 145, 1005-1032. 7

DOVis, A. AND R. KiRPALANi (2019a): "Fiscal rules, bailouts, and reputation in federal governments," Tech. rep., National Bureau of Economic Research. 6, 7, 29

(2019b): “Reputation, Bailouts, and Interest Rate Spread Dynamics," . 6

HALAC, M. AND P. YARED (2014): "Fiscal rules and discretion under persistent shocks," Econometrica, 82, 1557-1614. 6, 31

- (2017): “Fiscal Rules and Discretion in a World Economy," . 6

— (2018): “Fiscal Rules and Discretion under Self-Enforcement," . 6

Kareken, J. H. AND N. WAllace (1978): "Deposit insurance and bank regulation: A partial-equilibrium exposition," Journal of Business, 413-438. 3, 7, 9

KING, R. G., Y. K. LU, AND E. S. PASTEN (2008): “Managing expectations,” Journal of Money, Credit and banking, 40, 1625-1666. 7

KREPS, D. M. AND R. WiLSON (1982): "Reputation and imperfect information," Journal of economic theory, 27, 253-279. 6

Kydland, F. E. AND E. C. Prescott (1977): “Rules rather than discretion: The inconsistency of optimal plans," Journal of political economy, 85, 473-491. 2

LU, Y. K. (2013): “Optimal policy with credibility concerns," Journal of Economic Theory, 148, 2007-2032. 7

LU, Y. K., R. G. KING, AND E. PASTEN (2016): “Optimal reputation building in the New Keynesian model," Journal of Monetary Economics, 84, 233-249. 7

MARINOVIC, I. AND M. SzYDLOWSKI (2019): “Monitor Reputation and Transparency," . 7

Milgrom, P. AND J. RoberTS (1982): “Predation, reputation, and entry deterrence," Journal of economic theory, 27, 280-312. 6

NosAL, J. B. AND G. ORdOÑEZ (2016): “Uncertainty as commitment,” Journal of Monetary Economics, 80, 124-140. 3 
PHElan, C. (2006): "Public trust and government betrayal," Journal of Economic Theory, 130, 27-43. 6

Phelan, C. And E. Stacchetti (2001): "Sequential equilibria in a Ramsey tax model," Econometrica, 69, 1491-1518. 6

Piguillem, F. AND A. Riboni (2018): “Fiscal Rules as Bargaining Chips,” Tech. rep. 6

Piguillem, F. AND A. Schneider (2013): “Coordination, Efficiency and Policy Discretion," Tech. rep., Einaudi Institute for Economics and Finance (EIEF). 5

Prat, A. (2005): "The Wrong Kind of Transparency," The American Economic Review, 95, 862-877. 5

RoGOFF, K. (1985): "The optimal degree of commitment to an intermediate monetary target," The quarterly journal of economics, 100, 1169-1189. 6, 28, 29, 30

SANKTJOHANSER, A. (2018): “Optimally Stubborn,” Tech. rep., working paper. 7

VICKERS, J. (1986): “Signalling in a model of monetary policy with incomplete information," Oxford Economic Papers, 38, 443-455. 7 


\section{Appendix (Not for publication)}

\section{A Omitted proofs}

\section{A.1 Proof of Lemma 1}

Here we provide the proof for the case in which $w_{x}<0$. The case with $w_{x}>0$ follows from a symmetric argument.

Suppose first that $\pi_{\mathfrak{c}}(\rho)=\underline{\pi}$ for all $\rho$. We first prove a preliminary result.

Claim. Under Assumptions 1 and 2, if $w_{x}<0$ then $\pi^{*}(x)$ is increasing and convex and $\chi(\rho)$ is decreasing and convex.

Proof. The optimizing type's static best response, $\pi^{*}(x)$, is implicitly defined by the first order condition:

$$
w_{\pi}\left(x, \pi^{*}(x)\right)=0
$$

so by the implicit function theorem we have

$$
\pi_{x}^{*}(x)=-\frac{w_{x \pi}\left(x, \pi^{*}(x)\right)}{w_{\pi \pi}\left(x, \pi^{*}(x)\right)}>0
$$

where the inequality follows from $w_{x \pi}>0$ and $w_{\pi \pi}<0$.

Implicitly differentiating (23) twice we have

$$
\pi_{x x}^{*}(x)=\frac{w_{\pi \pi \pi}\left(x, \pi^{*}(x)\right) \pi_{x}^{*}(x)^{2}+2 w_{\pi \pi x} \pi_{x}^{*}(x)+w_{x \pi x}\left(x, \pi^{*}(x)\right)}{\left(-w_{\pi \pi}\left(x, \pi^{*}(x)\right)\right)}
$$

Thus, in order for $\pi^{*}(x)$ to be convex we need

$$
w_{\pi \pi \pi}\left(x, \pi^{*}(x)\right) \pi_{x}^{*}(x)^{2}+2 w_{\pi \pi x} \pi_{x}^{*}(x)+w_{x \pi x}\left(x, \pi^{*}(x)\right) \geqslant 0 .
$$

Notice that the expression above can be equivalently written as

$$
\left[\pi_{\mathrm{x}}^{*}(\mathrm{x}), 1\right] \nabla^{2} w_{\pi}(\mathrm{x}, \pi)\left[\begin{array}{c}
\pi_{\mathrm{x}}^{*}(\mathrm{x}) \\
1
\end{array}\right]
$$

which is positive since $w_{\pi}(x, \pi)$ is convex by Assumption 2. Hence $\pi^{*}(x)$ is increasing and convex.

Consider now

$$
\chi(\rho)=\phi\left(\rho \pi_{c}+(1-\rho) \pi_{0}(\rho)\right)
$$


where $\pi_{0}(\rho)=\pi^{*}(\chi(\rho))$. So

$$
\chi^{\prime}(\rho)=\frac{\phi^{\prime}\left[\pi_{\mathrm{c}}-\pi_{\mathrm{o}}\right]}{\left[1-\phi^{\prime}(1-\rho) \pi_{\mathrm{x}}^{*}(\chi(\rho))\right]}<0
$$

which is negative since $\pi_{0} \geqslant \underline{\pi}$ and $1-\phi^{\prime}(1-\rho) \pi_{x}^{*}(x) \geqslant 0$, where the latter follows from condition 3 of Assumption 2. Twice differentiating the implementability condition (25) we obtain

$$
\begin{aligned}
\chi^{\prime \prime}(\rho) & =\phi^{\prime \prime}\left[\pi_{\mathrm{c}}-\pi_{\mathrm{o}}+(1-\rho) \pi_{\mathrm{o}}^{\prime}(\rho)\right]^{2}+\phi^{\prime}\left[-\pi_{\mathrm{o}}^{\prime}(\rho)+(1-\rho) \pi_{\mathrm{o}}^{\prime \prime}(\rho)-\pi_{\mathrm{o}}^{\prime}(\rho)\right] \\
& =\phi^{\prime \prime}\left[\pi_{\mathrm{c}}-\pi_{\mathrm{o}}+(1-\rho) \pi_{\mathrm{o}}^{\prime}(\rho)\right]^{2}+\phi^{\prime}\left[(1-\rho) \pi_{\mathrm{o}}^{\prime \prime}(\rho)-2 \pi_{\mathrm{x}}^{*}(x) x^{\prime}(\rho)\right]
\end{aligned}
$$

and since $\pi_{0}(\rho)=\pi^{*}(x(\rho))$ then

$$
\pi_{\mathrm{o}}^{\prime \prime}(\rho)=\pi_{x x}^{*}(x) \chi^{\prime}(\rho)^{2}+\pi_{x}^{*}(x) \chi^{\prime \prime}(\rho)
$$

Therefore, using (28) to substitute for $\pi_{0}^{\prime \prime}(\rho)$ in (27), we obtain

$$
\chi^{\prime \prime}(\rho)=\frac{\phi^{\prime \prime}\left[\pi_{c}-\pi_{0}+(1-\rho) \pi_{o}^{\prime}(\rho)\right]^{2}+\left[\phi^{\prime}(1-\rho) \pi_{x x}^{*}(x) x^{\prime}(\rho)^{2}-2 \phi^{\prime} \pi_{x}^{*}(x) x^{\prime}(\rho)\right]}{\left[1-\phi^{\prime}(1-\rho) \pi_{x}^{*}(x)\right]}
$$

Thus $x^{\prime \prime} \geqslant 0$ follows from Assumption $1, \phi^{\prime} \geqslant 0, \phi^{\prime \prime} \geqslant 0, \pi_{x}^{*}(x)>0$, and $\pi_{x x}^{*}(x) \geqslant 0$, where the last two inequalities were proved earlier.

We now turn the proof of the lemma. Define

$$
\begin{gathered}
\bar{w}(x)=w\left(x, \pi_{c}\right) \\
w^{*}(x)=w\left(x, \pi^{*}(x)\right)
\end{gathered}
$$

and

$$
F(\rho, x(\rho))=\rho \bar{w}(x(\rho))+(1-\rho) w^{*}(x(\rho))
$$

and so $W_{0}(\rho)=F(\rho, \chi(\rho))$. We want to show that $W_{0}(\rho)$ is concave. We have,

$$
\begin{aligned}
W_{0}^{\prime \prime}(\rho) & =\left[1, x^{\prime}(\rho)\right] \nabla^{2} F(\rho, x(\rho))\left[\begin{array}{c}
1 \\
x^{\prime}(\rho)
\end{array}\right]+\nabla F(\rho, x(\rho))\left[\begin{array}{c}
0 \\
x^{\prime \prime}(\rho)
\end{array}\right] \\
& =2 F_{\rho x} x^{\prime}(\rho)+F_{x x} x^{\prime}(\rho)^{2}+F_{x}(\rho, x(\rho)) x^{\prime \prime}(\rho)
\end{aligned}
$$

Thus to prove the result it is sufficient to show that the above expression is negative. We proceed in several steps. First we show that $F_{x x} x^{\prime}(\rho)^{2}<0$ and then we show that 
$2 F_{\rho x} x^{\prime}(\rho)+F_{x}(\rho, x(\rho)) x^{\prime \prime}(\rho) \leqslant 0$.

To see that $F_{x x} x^{\prime}(\rho)^{2}<0$, note that

$$
F_{x}=\rho w_{x}\left(x, \pi_{c}\right)+(1-\rho) w_{x}\left(x, \pi_{0}\right)<0
$$

since $w_{x \pi}>0$, and so

$$
\begin{aligned}
F_{x x} & =\rho \bar{w}_{x x}(x(\rho))+(1-\rho) w_{x x}^{*}(x(\rho)) \\
& =\rho w_{x x}\left(x, \pi_{c}\right)+(1-\rho)\left[w_{x x}\left(x, \pi_{0}(x)\right)+w_{x \pi}(x, \pi) \pi_{x}^{*}(x)\right]
\end{aligned}
$$

The first term in (32) is negative since $w_{x x}<0$. For the second, note that from (24), we have that

$$
\pi_{x}^{*}(x)=-\frac{w_{x \pi}\left(x, \pi_{0}\right)}{w_{\pi \pi}\left(x, \pi_{0}\right)}
$$

Therefore, using (33) we can write the second term in (32) as

$$
w_{x x}\left(x, \pi_{0}\right)+w_{x \pi}\left(x, \pi_{0}\right) \pi_{x}^{*}(x)=\frac{w_{x x}\left(x, \pi_{0}\right) w_{\pi \pi}\left(x, \pi_{0}\right)-w_{x \pi}\left(x, \pi_{0}\right)^{2}}{w_{\pi \pi}\left(x, \pi_{0}\right)}<0
$$

where the inequality follows from $w$ being concave in $(x, \pi)$. Therefore, both terms in (32) are negative and so $F_{x x} x^{\prime}(\rho)^{2}<0$.

Now note that we can write $F_{\rho x}$ as

$$
\begin{aligned}
\mathrm{F}_{\rho x} & =\bar{w}_{x}(x(\rho))-w_{x}^{*}(x(\rho))=w_{x}\left(x, \pi_{c}\right)-\left[w_{x}\left(x, \pi^{*}(x)\right)+w_{\pi}\left(x, \pi^{*}(x)\right) \pi_{x}^{*}(x)\right] \\
& =w_{x}\left(x, \pi_{c}\right)-w_{x}\left(x, \pi^{*}(x)\right)<0
\end{aligned}
$$

Then from (30) it follows that

$$
\begin{aligned}
W_{0}^{\prime \prime}(\rho) & =2 F_{\rho x} x^{\prime}(\rho)+F_{x x} x^{\prime}(\rho)^{2}+F_{x}(\rho, x(\rho)) x^{\prime \prime}(\rho) \\
& <2 F_{\rho x} x^{\prime}(\rho)+F_{x}(\rho, x(\rho)) x^{\prime \prime}(\rho) \\
& =2\left[w_{x}\left(x, \pi_{c}\right)-w_{x}\left(x, \pi^{*}(x)\right)\right] x^{\prime}(\rho)+\left[\rho w_{x}\left(x, \pi_{c}\right)+(1-\rho) w_{x}\left(x, \pi^{*}(x)\right)\right] x^{\prime \prime}(\rho)
\end{aligned}
$$

where the first inequality follows from the fact that $F_{x x} x^{\prime}(\rho)^{2}<0$ and the second equality follows from (34) and (31). A sufficient condition for the concavity of $W_{0}$ is then

$$
2\left[w_{x}\left(x, \pi_{c}\right)-w_{x}\left(x, \pi^{*}(x)\right)\right] x^{\prime}(\rho)+\left[\rho w_{x}\left(x, \pi_{c}\right)+(1-\rho) w_{x}\left(x, \pi^{*}(x)\right)\right] x^{\prime \prime}(\rho) \leqslant 0
$$

or, rearranging terms,

$$
\frac{2\left[w_{x}\left(x, \pi^{*}(x)\right)-w_{x}\left(x, \pi_{c}\right)\right]}{-\left[\rho w_{x}\left(x, \pi_{c}\right)+(1-\rho) w_{x}\left(x, \pi^{*}(x)\right)\right]} \leqslant-\frac{\chi^{\prime \prime}(\rho)}{\chi^{\prime}(\rho)} .
$$


Using the expressions for $x^{\prime}(\rho)$ and $x^{\prime \prime}(\rho)$ in (26) and (29) we have

$$
\begin{aligned}
-\frac{\chi^{\prime \prime}(\rho)}{\chi^{\prime}(\rho)} & =\frac{\phi^{\prime \prime}\left[\pi_{c}-\pi_{0}+(1-\rho) \pi_{\mathrm{o}}^{\prime}(\rho)\right]^{2}+\left[\phi^{\prime}(1-\rho) \pi_{x x}^{*}(x) \chi^{\prime}(\rho)^{2}-2 \phi^{\prime} \pi_{x}^{*}(x) \chi^{\prime}(\rho)\right]}{\phi^{\prime}\left[\pi_{0}-\pi_{c}\right]} \\
& \geqslant \frac{-2 \phi^{\prime} \pi_{x}^{*}(x)}{\phi^{\prime}\left[\pi_{0}-\pi_{c}\right]} \chi^{\prime}(\rho) \\
& =\frac{2 \phi^{\prime} \pi_{x}^{*}(x)}{\left[1-\phi^{\prime}(1-\rho) \pi_{x}^{*}(x(\rho))\right]}
\end{aligned}
$$

where the first inequality follows from the fact that $\phi^{\prime \prime}>0, \phi^{\prime}>0, \pi_{x x}^{*}>0$ and the denominator is positive, and the last equality follows from using (26) to substitute for $x^{\prime}(\rho)$. Hence a sufficient condition for (35) is

$$
\frac{2\left[w_{x}\left(x, \pi^{*}(x)\right)-w_{x}\left(x, \pi_{c}\right)\right]}{-\left[\rho w_{x}\left(x, \pi_{c}\right)+(1-\rho) w_{x}\left(x, \pi^{*}(x)\right)\right]} \leqslant \frac{2 \pi_{x}^{*}(x) \phi^{\prime}}{\left[1-\phi^{\prime}(1-\rho) \pi_{x}^{*}(x)\right]}
$$

or, rearranging terms,

$$
\pi_{x}^{*}(x) \phi^{\prime}+\frac{w_{\chi}\left(x, \pi^{*}(x)\right)}{w_{x}\left(x, \pi_{c}\right)} \geqslant 1
$$

Notice that

$$
\pi_{x}^{*}(x) \phi^{\prime}+\frac{w_{\chi}\left(x, \pi^{*}(x)\right)}{w_{x}\left(x, \pi_{c}\right)} \geqslant \pi_{x}^{*}(\underline{x}) \phi^{\prime}(\underline{\pi})+\frac{w_{x}\left(\underline{x}, \pi^{*}(\underline{x})\right)}{w_{x}(\underline{x}, \underline{\pi})} \geqslant 1
$$

where we have used the fact that $\pi^{*}(x), \phi(\pi)$ are convex, $w(x, \pi)$ is concave, and the last inequality follows from condition 3 of Assumption 2. Thus $W_{0}^{\prime \prime}(\rho)<0$.

As a final step we will show that our assumptions imply that $\pi_{\mathrm{c}}$ is independent of $\rho$ and in particular equals $\underline{\pi}$. The first order condition of the static government's problem wrt $\pi_{\mathrm{c}}$ is

$$
\rho w_{\pi}(x, \pi)+\left[\rho w_{x}(x, \pi)+(1-\rho) w_{\chi}\left(x, \pi^{*}(x)\right)\right] \frac{\rho \phi^{\prime}(\cdot)}{\left[1-\phi^{\prime}(\cdot)(1-\rho) \pi_{x}^{*}(x)\right]}
$$

Since by assumption

$$
w_{\pi}(x, \pi)+\left[\rho w_{\chi}(x, \pi)+(1-\rho) w_{\chi}\left(x, \pi^{*}(x)\right)\right] \frac{\phi^{\prime}(\cdot)}{\left[1-\phi^{\prime}(\cdot)(1-\rho) \pi_{x}^{*}(x)\right]} \leqslant 0
$$

it must be that $\pi_{\mathrm{c}}=\underline{\pi}$. Q.E.D.

We next show that the two examples satisfy Assumption 2.

Lemma 2. The Barro-Gordon economy satisfies Assumption 2. 
Proof. Recall that

$$
w(x, \pi)-\frac{1}{2}\left[(\psi+x-\pi)^{2}+\pi^{2}\right]
$$

Thus,

$$
\begin{aligned}
& w_{x x}=-1<0 \\
& w_{\pi \pi}=-2<0
\end{aligned}
$$

and

$$
w_{x x} w_{\pi \pi}-w_{x \pi}=1>0
$$

Therefore the Hessian of $w(x, \pi)$ is negative semi-definite and thus $w(x, \pi)$ is concave.

Next, note that $w_{\pi \pi \mathrm{a}}=0, w_{\pi \pi \pi}=0$ and $w_{\pi \mathrm{aa}}=0$ and so $w_{\pi}$ is convex.

Next, since

$$
\pi^{*}(x)=\frac{x+\psi}{2}
$$

we have

$$
\pi_{x}^{*}(x) \phi^{\prime}(\pi)=\frac{1}{2}
$$

Therefore

$$
1>\pi_{x}^{*}(\mathrm{x}) \phi^{\prime}(\pi)=\frac{1}{2} \geqslant 1-\frac{w_{\chi}\left(\underline{x}, \pi^{*}(\underline{x})\right)}{w_{x}(\underline{x}, \underline{\pi})}=\frac{1}{2}
$$

and so condition 3 is satisfied. Finally, using a little algebra condition 4 is

$$
\left[-2+\frac{2 \rho}{1+\rho}\right] \pi_{\mathrm{c}}
$$

which is strictly less than zero for any $\pi_{\mathrm{c}}$ positive. Thus, it must be $\pi_{\mathrm{c}}=0$. Q.E.D.

Lemma 3. If $\psi$ is sufficiently small, then bailout economy satisfies Assumption 2.

Proof. Recall that

$$
w(e, \pi)=-v(e)+p(e) R_{H}-(1-p(e))(1-\pi) \psi-c(\pi)
$$

and thus $\pi^{*}(e)$ is the solution to

$$
(1-p(e)) \psi-c^{\prime}(\pi)=0
$$

Let's first show that $w(e, \pi)$ is concave. We have

$$
\begin{gathered}
w_{e}=-v^{\prime}(e)+p^{\prime}(e)\left(R_{H}+(1-\pi) \psi\right) \\
w_{e e}=-v^{\prime \prime}(e)+p^{\prime \prime}(e)\left(R_{H}+(1-\pi) \psi\right)<0
\end{gathered}
$$




$$
\begin{gathered}
w_{e \pi}=-p^{\prime \prime}(e) \psi>0 \\
\mathcal{w}_{\pi}=(1-p(e)) \psi-c^{\prime}(\pi) \\
w_{\pi \pi}=-c^{\prime \prime}(\pi) \leqslant 0
\end{gathered}
$$

So

$$
\begin{aligned}
& \mathcal{w}_{e e} \mathcal{W}_{\pi \pi}-w_{e \pi}^{2} \\
= & {\left[-v^{\prime \prime}(e)+p^{\prime \prime}(e)\left(R_{H}+(1-\pi) \psi\right)\right]\left(-c^{\prime \prime}(\pi)\right)-p^{\prime \prime}(e)^{2} \psi^{2} }
\end{aligned}
$$

The first term is positive since $v^{\prime \prime}>0, p^{\prime \prime}<0$, and $c^{\prime \prime}>0$ but $-p^{\prime \prime}(e)^{2} \psi^{2}$ is negative. Clearly, the whole expression is positive if $\psi$ is small enough. Thus the Hessian of $w$ is negative semi-definite which implies that $w$ is concave.

Next, we show that $w_{\pi}(e, \pi)$ is convex. We have

$$
w_{\pi}(e, \pi)=(1-p(e)) \psi-c^{\prime}(\pi)
$$

Therefore

$$
\begin{gathered}
w_{\pi e}=-p^{\prime}(e) \psi \\
w_{\pi e e}=-p^{\prime \prime}(e) \psi>0 \\
w_{\pi e \pi}=0 \\
w_{\pi \pi \pi}=-c^{\prime \prime \prime}(\pi)=0
\end{gathered}
$$

since $c(e)$ is quadratic and so $c^{\prime \prime \prime}=0$. Therefore

$$
w_{\pi e e} \mathcal{w}_{\pi \pi \pi}-w_{\pi e \pi}^{2}=\left[-p^{\prime}(e) \psi\right]\left[-c^{\prime \prime \prime}(e)\right]=0
$$

Thus the Hessian of $\mathcal{w}_{\pi}$ is positive semi-definite and so $\boldsymbol{w}_{\pi}$ is convex.

We now check that condition 3 is satisfied. We have to show that the following two conditions hold:

$$
\begin{gathered}
1>\pi_{x}^{*}(x) \phi^{\prime}(\pi) \\
\pi_{x}^{*}(x) \phi^{\prime}(\pi) \geqslant 1-\frac{w_{x}\left(\underline{x}, \pi^{*}(\underline{x})\right)}{w_{x}(\underline{x}, \underline{\pi})}
\end{gathered}
$$

Notice that

$$
\pi_{x}^{*}(x) \phi^{\prime}(\pi)=\left(-\frac{p^{\prime}(e) \psi}{c^{\prime \prime}(\pi)}\right) \phi^{\prime}(\pi)
$$


and

$$
1-\frac{w_{\chi}\left(\underline{\chi}, \pi^{*}(\underline{x})\right)}{w_{\chi}(\underline{x}, \underline{\pi})}=1-\frac{-v^{\prime}(\underline{e})+p^{\prime}(\underline{e}) R_{H}+p^{\prime}(\underline{e})\left(1-\pi^{*}(\underline{e})\right) \psi}{-v^{\prime}(\underline{e})+p^{\prime}(\underline{e}) R_{H}+p^{\prime}(\underline{e}) \psi}=\pi^{*}(\underline{e}) .
$$

Thus, the two conditions can be written as

$$
1>\left(-\frac{p^{\prime}(e) \psi}{c^{\prime \prime}(\pi)}\right) \phi^{\prime}(\pi)
$$

and

$$
\left(-\frac{p^{\prime}(e) \psi}{c^{\prime \prime}(\pi)}\right) \phi^{\prime}(\pi) \geqslant \pi^{*}(\underline{e}) .
$$

Under our functional form assumptions,

$$
\begin{gathered}
p(e)=e^{\alpha} \\
c(\pi)=\lambda \pi^{2} / 2 \\
v(e)=e^{2} / 2,
\end{gathered}
$$

we have that

$$
e=\phi(\pi)=\alpha^{\eta}\left(R_{H}-\pi\right)^{\eta}, \quad \eta \equiv 1 /(2-\alpha) \in(0,1)
$$

so

$$
\begin{aligned}
\left(-\frac{p^{\prime}(e) \psi}{c^{\prime \prime}(\pi)}\right) \phi^{\prime}(\pi) & =\frac{-\alpha e^{\alpha-1} \psi}{\lambda} \eta \alpha^{\eta}\left(R_{H}-\pi\right)^{\eta-1} \\
& =\frac{-\alpha\left(\alpha^{\eta}\left(R_{H}-\pi\right)^{\eta}\right)^{\alpha-1} \psi}{\lambda}\left(-\eta \alpha^{\eta}\left(R_{H}-\pi\right)^{\eta-1}\right) \\
& =\frac{\alpha^{1+\eta \alpha}}{2-\alpha} \frac{1}{\left(R_{H}-\pi\right)^{1-\eta \alpha}} \frac{\psi}{\lambda}
\end{aligned}
$$

and since $\underline{e}=\alpha^{\eta} R_{H}^{\eta}$ we have that

$$
\pi^{*}(\underline{e})=\frac{1-p\left(\alpha^{\eta} R_{H}^{\eta}\right)}{\lambda} \psi=\left[1-\left(\alpha R_{H}\right)^{\eta \alpha}\right] \frac{\psi}{\lambda} .
$$

By inspection, the first inequality, (37), is satisfied if $\psi$ is sufficiently small while the second inequality, (38), is satisfied if $R_{H}$ is sufficiently large. In fact, as $R_{H} \rightarrow 1 / \alpha, p(e) \rightarrow 1$ so $\pi^{*}(\underline{e}) \rightarrow 0$ while $\left(-\frac{p^{\prime}(e) \psi}{c^{\prime \prime}(\pi)}\right) \phi^{\prime}(\pi)>0$. 
Finally, we check that condition 4 holds. We have

$$
\begin{aligned}
& w_{\pi}(e, \pi)+\left[\rho w_{e}(e, \pi)+(1-\rho) w_{e}\left(e, \pi^{*}(e)\right)\right] \frac{\phi^{\prime}(\cdot)}{\left[1-\phi^{\prime}(\cdot)(1-\rho) \pi_{e}^{*}(e)\right]} \\
= & (1-p(e)) \psi-c^{\prime}(\pi) \\
& +\left[\rho p^{\prime}(e)[\pi+(1-\pi) \psi]+(1-\rho)\left[\pi_{0}(e)+\left(1-\pi^{*}(e)\right) \psi\right]\right] \frac{\phi^{\prime}(\cdot)}{\left[1-\phi^{\prime}(\cdot)(1-\rho)\left(-\frac{p^{\prime}(e) \psi}{c^{\prime \prime}(\pi)}\right)\right]}
\end{aligned}
$$

which is negative if $\psi$ is sufficiently small since $c^{\prime}>0, w_{e} \geqslant 0, \phi^{\prime} \leqslant 0$, and $1-\phi^{\prime} \pi_{e}^{*} \geqslant 0$. Q.E.D.

\section{A.2 Optimizing type does not randomize}

We now show that under our assumptions it is without loss of generality to consider the case in which the optimizing type either follows the rule with probability one or chooses its best response and deviates from the rule with probability one.

To see this, let's allow the allow the optimizing type to randomize. The value the rule designer can attain by inducing the optimizing type to follow the rule with probability $\sigma$ starting with a prior $\rho$ is

$$
\begin{aligned}
W_{\text {pool }}(\sigma, \rho) & =[\rho+(1-\rho) \sigma]\left[w\left(x_{\text {ico }}(\sigma, \rho), \pi_{\text {ico }}(\sigma, \rho)\right)+\beta W_{0}\left(\rho^{\prime}(\rho, \sigma)\right)\right] \\
& +(1-\rho)(1-\sigma)\left[w\left(x_{\text {ico }}(\sigma, \rho), \pi^{*}\left(x_{\text {ico }}(\sigma, \rho)\right)\right)+\beta W_{0}(0)\right]
\end{aligned}
$$

where the evolution of the prior is given by

$$
\rho^{\prime}(\sigma, \rho)=\frac{\rho}{\rho+(1-\rho) \sigma}
$$

and $\left(x_{\text {ico }}(\sigma, \rho), \pi_{\text {ico }}(\sigma, \rho)\right)$ solves

$$
x_{\text {ico }}(\sigma, \rho)=\phi\left([\rho+(1-\rho) \sigma] \pi_{\text {ico }}(\sigma, \rho)+(1-\rho)(1-\sigma) \pi^{*}\left(x_{\text {ico }}(\sigma, \rho)\right)\right)
$$

and

$$
w\left(x_{\text {ico }}(\sigma, \rho), \pi_{\text {ico }}(\sigma, \rho)\right)+\beta V_{0}\left(\rho^{\prime}(\rho, \sigma)\right)=w\left(x_{\text {ico }}(\sigma, \rho), \pi^{*}\left(x_{\text {ico }}(\sigma, \rho)\right)\right)+\beta V_{0}(0)
$$

That is, $\pi_{\text {ico }}(\sigma, \rho)$ is the most stringent policy that is incentive compatible for the optimizing type given $\rho$ and $\sigma$.

We next show that it is optimal to choose $\sigma=1$. We will use the following intermediate 
result:

Lemma 4. Under Assumptions 1 and 2, $\mathrm{V}_{0}(\rho)$ is concave.

Proof. We prove this for the case with $w_{x}<0$. The proof for the other case is identical. Recall that

$$
V_{0}(\rho)=w\left(x(\rho), \pi^{*}(x(\rho))\right)
$$

Then

$$
\begin{aligned}
V_{0}^{\prime}(\rho) & =w_{\chi} \chi^{\prime}(\rho)+w_{\pi} \pi_{x}^{*}(x) \chi^{\prime}(\rho) \\
& =w_{\chi} \chi^{\prime}(\rho)
\end{aligned}
$$

where we used that $w_{\pi}\left(x, \pi^{*}(x)\right)=0$. Then

$$
\begin{aligned}
V^{\prime \prime}(\rho) & =\left(w_{x x}+w_{x \pi} \pi_{x}^{*}\right) x^{\prime}(\rho)^{2}+w_{x} x^{\prime \prime}(\rho) \\
& =\left(\frac{w_{x x} w_{\pi \pi}\left(x, \pi^{*}\right)-w_{x \pi}\left(x, \pi^{*}\right)^{2}}{w_{\pi \pi}\left(x, \pi_{0}\right)}\right) x^{\prime}(\rho)^{2}+w_{x} x^{\prime \prime}(\rho) \\
& <0
\end{aligned}
$$

where the second line follows from using (24) to substitute for $\pi_{x}^{*}$ and the last inequality follows from Assumption 2 and $x^{\prime \prime}(\rho) \geqslant 0$ where the latter property was established in the proof of Lemma 1. Q.E.D.

Lemma 5. Under Assumptions 1 and 2 , for all $\rho$ and $\sigma, W_{\text {pool }}(1, \rho) \geqslant W_{\text {pool }}(\sigma, \rho)$.

Proof. Consider $\sigma<1$. Consider a deviation in which the optimizing type chooses the following policy

$$
\pi_{\mathrm{dev}}=[\rho+(1-\rho) \sigma] \pi_{\mathrm{ico}}(\sigma, \rho)+(1-\rho)(1-\sigma) \pi^{*}\left(x_{\mathrm{ico}}(\sigma, \rho)\right)
$$

with probability one and $\pi_{\mathrm{c}}=\pi_{\mathrm{dev}}$. Note that this policy is just the expected value of the policies followed by the optimizing type when there is randomization. Therefore, under this deviation, $x_{\text {ico }}(\sigma, \rho)$ is unaffected. Since $w$ is concave in $\pi$ and $W_{0}$ is concave in $\rho$, this policy improves welfare:

$$
\begin{aligned}
W_{\text {pool }}(\sigma, \rho) & =[\rho+(1-\rho) \sigma] w\left(x_{\text {ico }}(\sigma, \rho), \pi_{\text {ico }}(\sigma, \rho)\right)+(1-\rho)(1-\sigma) w\left(x_{\text {ico }}(\sigma, \rho), \pi^{*}\left(x_{\text {ico }}(\sigma, \rho)\right)\right) \\
& +[\rho+(1-\rho) \sigma] \beta W_{0}\left(\rho^{\prime}(\rho, \sigma)\right)+(1-\rho)(1-\sigma) \beta W_{0}(0) \\
& \leqslant w\left(x_{i c o}(\sigma, \rho),[\rho+(1-\rho) \sigma] \pi_{\text {ico }}(\sigma, \rho)+(1-\rho)(1-\sigma) \pi^{*}\left(x_{\text {ico }}(\sigma, \rho)\right)\right)+\beta W_{0}(\rho) \\
& =w\left(x_{\text {ico }}(\sigma, \rho), \pi_{\text {dev }}\right)+\beta W_{0}(\rho)
\end{aligned}
$$


We are left to show that this deviation is feasible for the rule designer in that it satisfies the incentive compatibility constraint for the optimizing type:

$$
w\left(x_{i c o}(\sigma, \rho), \pi_{\text {dev }}\right)+\beta V_{0}(\rho) \geqslant w\left(x_{i c o}(\sigma, \rho), \pi^{*}\left(x_{i c o}(\sigma, \rho)\right)\right)+\beta V_{0}(0)
$$

Note that at the original allocation it must be that

$$
w\left(x_{\text {ico }}(\sigma, \rho), \pi_{\text {ico }}(\sigma, \rho)\right)+\beta V_{0}\left(\rho^{\prime}(\rho, \sigma)\right) \geqslant w\left(x_{i c o}(\sigma, \rho), \pi^{*}\left(x_{i c o}(\sigma, \rho)\right)\right)+\beta V_{0}(0)
$$

and trivially

$$
w\left(x_{i c o}(\sigma, \rho), \pi^{*}\left(x_{i c o}(\sigma, \rho)\right)\right)+\beta V_{0}(0) \geqslant w\left(x_{i c o}(\sigma, \rho), \pi^{*}\left(x_{i c o}(\sigma, \rho)\right)\right)+\beta V_{0}(0)
$$

Multiplying the left and right side of (39) by $[\rho+(1-\rho) \sigma]$, the left and right side of (40) by $(1-\rho)(1-\sigma)$, and summing up the two resulting equations yields

$$
\begin{aligned}
w\left(x_{i c o}(\sigma, \rho), \pi^{*}\left(x_{i c o}(\sigma, \rho)\right)\right)+\beta V_{0}(0) & \leqslant[\rho+(1-\rho) \sigma]\left[w\left(x_{i c o}(\sigma, \rho), \pi_{i c o}(\sigma, \rho)\right)+\beta V_{0}\left(\rho^{\prime}(\rho, \sigma)\right)\right] \\
& +(1-\rho)(1-\sigma)\left[w\left(x_{i c o}(\sigma, \rho), \pi^{*}\left(x_{i c o}(\sigma, \rho)\right)\right)+\beta V_{0}(0)\right] \\
& \leqslant w\left(x_{i c o}(\sigma, \rho), \pi_{\text {dev }}\right)+\beta V_{0}(\rho)
\end{aligned}
$$

where the second inequality follows from concavity of $w$ in $\pi$ and $V_{0}$ in $\rho$. Thus the proposed deviation is incentive compatible and it increases welfare. Moreover, since

$$
W_{\text {pool }}(1, \rho)=\max _{\pi_{\mathrm{c}}} w\left(\phi\left(\pi_{\mathrm{c}}\right), \pi_{\mathrm{c}}\right)+\beta W_{0}(\rho)
$$

subject to

$$
w\left(\phi\left(\pi_{\mathrm{c}}\right), \pi_{\mathrm{c}}\right)+\beta \mathrm{V}_{0}(\rho) \geqslant w\left(\phi\left(\pi_{\mathrm{c}}\right), \pi^{*}\left(\phi\left(\pi_{\mathrm{c}}\right)\right)\right)+\beta \mathrm{V}_{0}(0)
$$

and since $\pi_{\mathrm{dev}}$ is feasible for this problem we have

$$
W_{\text {pool }}(1, \rho) \geqslant w\left(x_{\text {ico }}(\sigma, \rho), \pi_{\text {dev }}\right)+\beta W_{0}(\rho) \geqslant W_{\text {pool }}(\sigma, \rho)
$$

as wanted. Q.E.D.

\section{A.3 Proof of Proposition 1}

Consider first $\rho$ close to 1 . Since the incentive compatibility is binding, we have that for some $\delta>0$, for all $\rho$

$$
W_{\text {ramsey }}-\delta \geqslant W_{\text {pool }}(\rho)
$$


Clearly, at $\rho=1, W_{\text {sep }}(1)$ attains the Ramsey outcome. By continuity, there exists a $\varepsilon_{\delta}$ sufficiently small such that for all $\rho \in\left(1-\varepsilon_{\delta}, 1\right)$,

$$
W_{\text {sep }}(\rho) \geqslant W_{\text {ramsey }}-\delta
$$

Combining the two expressions above we have that for all $\rho \in\left(1-\varepsilon_{\delta}, 1\right)$,

$$
W_{\text {pool }}(\rho)<W_{\text {sep }}(\rho)
$$

as wanted.

Consider now $\rho$ close to zero and assume that Assumptions 1 and 2 hold. Thus by Lemma 1 uncertainty is beneficial, $W_{0}(\rho)>\rho W_{0}(1)+(1-\rho) W_{0}(0)$, and the continuation value is higher under pooling than under the separation policy. To show that it is optimal to pool, it is sufficient to show that the static benefits of pooling are positive for priors close to zero, i.e. $\Delta \omega(\rho) \geqslant 0$ where

$$
\Delta \omega(\rho)=w\left(\phi\left(\pi_{\text {ico }}(\rho)\right), \pi_{\text {ico }}(\rho)\right)-W_{0}(\rho)
$$

and

$$
W_{0}(\rho)=\left[\rho w\left(\phi\left(\rho \pi_{c}+(1-\rho) \pi_{0}\right), \pi_{c}\right)+(1-\rho) w\left(\phi\left(\rho \pi_{c}+(1-\rho) \pi_{0}\right), \pi_{0}\right)\right]
$$

To this end, note that at $\rho=0$ we have $\Delta \omega(0)=0$ since $\pi_{\text {ico }}(0)=\pi_{0}(0)=\pi^{*}\left(\phi\left(\pi_{0}(0)\right)\right)$. Thus it is sufficient to show that $\Delta \omega^{\prime}(0)>0$. Note that

$$
\begin{aligned}
W_{0}^{\prime}(\rho) & =\mathcal{w}\left(\phi\left(\rho \pi_{c}+(1-\rho) \pi_{\mathrm{o}}\right), \pi_{\mathrm{c}}\right)-\mathcal{w}\left(\phi\left(\rho \pi_{\mathrm{c}}+(1-\rho) \pi_{\mathrm{o}}\right), \pi_{\mathrm{o}}\right) \\
& +\left[\rho w_{x}\left(\phi\left(\rho \pi_{\mathrm{c}}+(1-\rho) \pi_{\mathrm{o}}\right), \pi_{\mathrm{c}}\right)+(1-\rho) \mathcal{w}_{x}\left(\phi\left(\rho \pi_{\mathrm{c}}+(1-\rho) \pi_{\mathrm{o}}\right), \pi_{\mathrm{c}}\right)\right] \phi^{\prime}\left(\rho \pi_{\mathrm{c}}+(1-\rho) \pi_{\mathrm{o}}\right) \\
& \times\left[\pi_{\mathrm{c}}-\pi_{\mathrm{o}}+(1-\rho) \pi_{\mathrm{o}}^{\prime}(\rho)\right]
\end{aligned}
$$

where we used that $w_{\pi}\left(\phi\left(\rho \pi_{c}+(1-\rho) \pi_{\mathrm{o}}\right), \pi_{\mathrm{o}}\right)=0$. Therefore,

$$
\begin{aligned}
\Delta \omega^{\prime}(0) & =\left[w_{x}\left(\phi\left(\pi_{i c o}(0)\right), \pi_{i c o}(0)\right) \phi^{\prime}\left(\pi_{i c o}(0)\right)+w_{\pi}\left(\phi\left(\pi_{i c o}(0)\right), \pi_{i c o}(0)\right)\right] \pi_{i c o}^{\prime}(0) \\
& -\left\{w\left(\phi\left(\pi_{\mathrm{o}}(0)\right), \pi_{\mathrm{c}}\right)-w\left(\phi\left(\pi_{\mathrm{o}}(0)\right), \pi_{\mathrm{o}}(0)\right)\right. \\
& \left.+w_{x}\left(\phi\left(\pi_{\mathrm{o}}(0)\right), \pi_{\mathrm{o}}(0)\right) \phi^{\prime}\left(\pi_{\mathrm{o}}(0)\right)\left[\pi_{\mathrm{c}}-\pi_{\mathrm{o}}+\pi_{\mathrm{o}}^{\prime}(0)\right]\right\} \\
& \geqslant\left[w_{x}\left(\phi\left(\pi_{i c o}(0)\right), \pi_{\mathrm{ico}}(0)\right) \phi^{\prime}\left(\pi_{i c o}(0)\right)+w_{\pi}\left(\phi\left(\pi_{i c o}(0)\right), \pi_{i c o}(0)\right)\right] \pi_{\mathrm{ico}}^{\prime}(0) \\
& -w_{x}\left(\phi\left(\pi_{\mathrm{o}}(0)\right), \pi_{\mathrm{o}}(0)\right) \phi^{\prime}\left(\pi_{\mathrm{o}}(0)\right)\left[\pi_{\mathrm{c}}-\pi_{\mathrm{o}}+\pi_{\mathrm{o}}^{\prime}(0)\right] \\
& =w_{x}\left(\phi\left(\pi_{i c o}(0)\right), \pi_{i c o}(0)\right) \phi^{\prime}\left(\pi_{i c o}(0)\right)\left[\pi_{i c o}^{\prime}(0)-\left(\pi_{\mathrm{c}}-\pi_{\mathrm{o}}\right)-\pi_{\mathrm{o}}^{\prime}(0)\right]
\end{aligned}
$$


where the first inequality follows from $w\left(\phi\left(\pi_{\mathrm{o}}(0)\right), \pi_{\mathrm{c}}\right)-w\left(\phi\left(\pi_{\mathrm{o}}(0)\right), \pi_{\mathrm{o}}(0)\right)<0$ and the last equality follows from $\pi_{\text {ico }}(0)=\pi_{\mathrm{o}}(0)$ which implies that $w_{\pi}\left(\phi\left(\pi_{\text {ico }}(0)\right), \pi_{\text {ico }}(0)\right)=$ 0 . Since $w_{x}<0$ and $\phi^{\prime}>0$ then it is sufficient to show that the term in square brackets is negative. To this end, we next show that $\pi_{\mathrm{ico}}^{\prime}(0)=-\infty$ and $-\left(\pi_{\mathrm{c}}-\pi_{\mathrm{o}}\right)-\pi_{\mathrm{o}}^{\prime}(0)$ is bounded.

Let's start with proving that $\lim _{\rho \rightarrow 0} \pi_{\text {ico }}^{\prime}(\rho)=-\infty$. Recall that $\pi_{\text {ico }}(\rho)$ is implicitly defined by the incentive compatibility constraint

$$
\mathcal{w}\left(\phi\left(\pi_{\text {ico }}(\rho)\right), \pi_{\text {ico }}(\rho)\right)+\beta V_{0}(\rho)=w\left(\phi\left(\pi_{\text {ico }}(\rho)\right), \pi^{*}\left(\phi\left(\pi_{\text {ico }}(\rho)\right)\right)\right)+\beta V_{0}(0)
$$

Totally differentiating and evaluating at $\rho=0$ we have that

$$
w_{\pi}\left(\phi\left(\pi_{i c o}(0)\right), \pi_{i c o}(0)\right) \pi_{i c o}^{\prime}(0)=-\beta V_{0}^{\prime}(0)
$$

where we used that $\pi_{\text {ico }}(0)=\pi^{*}\left(\phi\left(\pi_{\mathrm{o}}(0)\right)\right)$. Since

$$
\mathcal{w}_{\pi}\left(\phi\left(\pi_{\text {ico }}(0)\right), \pi_{\text {ico }}(0)\right)=\mathcal{w}_{\pi}\left(\phi\left(\pi_{\text {ico }}(0)\right), \pi_{\mathrm{o}}(0)\right)=0
$$

and $-\beta W_{0}^{\prime}(0)<0$, it must be that $\lim _{\rho \rightarrow 0} \pi_{\text {ico }}^{\prime}(\rho)=-\infty$ since $\mathcal{w}_{\pi} \geqslant 0$ in the relevant range.

Since $-\left(\pi_{c}-\pi_{0}\right) \leqslant \bar{\pi}-\underline{\pi}$, we are left to show that $\pi_{\mathrm{o}}^{\prime}(0)$ is bounded. Recall that $\pi_{\mathrm{o}}(\rho)$ is the solution to

$$
\mathcal{w}_{\pi}\left(\phi\left(\rho \pi_{\mathrm{c}}+(1-\rho) \pi_{\mathrm{o}}\right), \pi_{\mathrm{o}}\right)=0
$$

and so applying the implicit function theorem we have

$$
\begin{aligned}
\pi_{\mathrm{o}}^{\prime}(0) & =-\frac{\mathcal{w}_{\pi x}\left(\phi\left(\pi_{\mathrm{o}}(0)\right), \pi_{\mathrm{o}}(0)\right) \chi^{\prime}(0)}{\mathcal{w}_{\pi \pi}\left(\phi\left(\pi_{\mathrm{o}}(0)\right), \pi_{\mathrm{o}}(0)\right)} \\
& =-\frac{\mathcal{w}_{\pi x}\left(\phi\left(\pi_{\mathrm{o}}(0)\right), \pi_{\mathrm{o}}(0)\right)}{\mathcal{w}_{\pi \pi}\left(\phi\left(\pi_{\mathrm{o}}(0)\right), \pi_{\mathrm{o}}(0)\right)} \times \frac{\phi^{\prime}\left[\pi_{\mathrm{c}}-\pi_{\mathrm{o}}\right]}{\left[1-\phi^{\prime} \pi_{x}^{*}(\chi)\right]}
\end{aligned}
$$

Using (24) and (26) we can rewrite the expression above as

$$
\pi_{\mathrm{o}}^{\prime}(0)=\pi_{x}^{*}(x(0)) x^{\prime}(0)=\pi_{x}^{*}(x(0)) \frac{\phi^{\prime}\left[\pi_{\mathrm{c}}-\pi_{\mathrm{o}}\right]}{\left[1-\phi^{\prime} \pi_{\mathrm{x}}^{*}(x)\right]}
$$

So

$$
\left|\pi_{\mathrm{o}}^{\prime}(0)\right| \leqslant\left|\pi_{x}^{*}(x(0))\right| \frac{\left|\phi^{\prime}\left(\pi_{M}\right)\right|[\bar{\pi}-\underline{\pi}]}{\left[1-\phi^{\prime}\left(\pi_{M}\right) \pi_{x}^{*}\left(\phi\left(\pi_{M}\right)\right)\right]}<\infty
$$

since $\phi^{\prime}$ is assumed to be bounded and $1-\phi^{\prime} \pi_{x}^{*}(x)$ is generically not equal to zero. In particular, if the economy satisfies Assumption 2 then $\left[1-\phi^{\prime}\left(\pi_{M}\right) \pi_{x}^{*}\left(\phi\left(\pi_{M}\right)\right)\right]>0$. Suppose not. Then it must be that $\left[1-\phi^{\prime}\left(\pi_{M}\right) \pi_{x}^{*}\left(\phi\left(\pi_{M}\right)\right)\right] \leqslant 0$ which contradicts Assumption 2 
part 3. Thus $\pi_{0}^{\prime}(0)$ is bounded.

The above claims imply that $\left[\pi_{\text {ico }}^{\prime}(0)-\left(\pi_{\mathrm{c}}-\pi_{\mathrm{o}}\right)-\pi_{\mathrm{o}}^{\prime}(0)\right]<0$ so $\Delta \omega^{\prime}(0)>0$ as wanted. Q.E.D.

\section{A.4 Proof of Proposition 1 for the Barro-Gordon example}

Consider the Barro-Gordon example with

$$
\phi\left(\rho \pi_{c}+(1-\rho) \pi_{\mathrm{o}}\right)=\rho \pi_{\mathrm{c}}+(1-\rho) \pi_{\mathrm{o}}
$$

and

$$
w(\chi, \pi)=-\frac{1}{2}\left[(\psi+\chi-\pi)^{2}+\pi^{2}\right]
$$

Therefore,

$$
\begin{gathered}
w_{\pi}=-[-(\psi+x)+2 \pi] \\
w_{x}=-(\psi+x-\pi)
\end{gathered}
$$

Moreover, the static best response is

$$
\pi^{*}(x)=\frac{(\psi+x)}{2}
$$

Consider first the static problem:

$$
W_{0}(\rho)=\max _{\pi_{c}, \pi_{o}, x}-\rho \frac{1}{2}\left[\left(\psi+x-\pi_{c}\right)^{2}+\pi_{c}^{2}\right]-(1-\rho) \frac{1}{2}\left[\left(\psi+x-\pi_{o}\right)^{2}+\pi_{o}^{2}\right]
$$

subject to

$$
\begin{gathered}
x=\rho \pi_{c}+(1-\rho) \pi_{\mathrm{o}} \\
\pi_{\mathrm{o}}=\frac{(\psi+\chi)}{2}
\end{gathered}
$$

Combining the two constraints we can express $\pi_{\mathrm{o}}$ and $\mathrm{x}$ in terms of $\pi_{\mathrm{c}}$ as

$$
\pi_{\mathrm{o}}=\frac{\psi+\rho \pi_{\mathrm{c}}}{(1+\rho)}=\frac{\rho}{1+\rho} \pi_{\mathrm{c}}+\frac{\psi}{(1+\rho)}
$$

and

$$
x=\frac{2 \rho}{1+\rho} \pi_{c}+\frac{(1-\rho)}{(1+\rho)} \psi
$$


Therefore, substituting into the objective function we obtain

$$
\begin{aligned}
W_{0}(\rho) & =\max -\frac{1}{2}\left(\rho\left[\left(\psi+\frac{2 \rho}{1+\rho} \pi_{c}+\frac{(1-\rho)}{(1+\rho)} \psi-\pi_{c}\right)^{2}+\pi_{c}^{2}\right]\right. \\
& \left.+(1-\rho)\left[\left(\psi+\frac{2 \rho}{1+\rho} \pi_{c}+\frac{(1-\rho)}{(1+\rho)} \psi-\frac{\rho}{1+\rho} \pi_{c}-\frac{\psi}{(1+\rho)}\right)^{2}+\left(\frac{\rho}{1+\rho} \pi_{c}+\frac{\psi}{(1+\rho)}\right)^{2}\right]\right) \\
& =\max _{\pi_{\mathfrak{c}}}-\frac{1}{2}\left(\rho\left[\left(\frac{2}{(1+\rho)} \psi-\left(\frac{1-\rho}{1+\rho}\right) \pi_{c}\right)^{2}+\pi_{c}^{2}\right]+(1-\rho)\left[2\left(\frac{\psi}{(1+\rho)}+\frac{\rho}{1+\rho} \pi_{c}\right)^{2}\right]\right)
\end{aligned}
$$

the first order condition for the above problem is

$$
\begin{aligned}
0 & =\left[-\left(\left(\frac{1-\rho}{1+\rho}\right) \frac{4 \rho}{(1+\rho)} \psi-\rho\left(\frac{1-\rho}{1+\rho}\right) 2\left(\frac{1-\rho}{1+\rho}\right) \pi_{\mathrm{c}}\right)+2 \rho \pi_{\mathrm{c}}\right] \\
& +\left[4 \frac{\rho(1-\rho)}{1+\rho}\left(\frac{\psi}{(1+\rho)}+\frac{\rho}{1+\rho} \pi_{\mathrm{c}}\right)\right]
\end{aligned}
$$

which implies that

$$
\pi_{\mathrm{c}}=0
$$

Therefore

$$
\begin{aligned}
& \pi_{0}(\rho)=\frac{\psi}{(1+\rho)^{\prime}}, \\
& \chi(\rho)=\frac{(1-\rho)}{(1+\rho)} \psi
\end{aligned}
$$

and

$$
W_{0}(\rho)=-\frac{\psi^{2}}{(1+\rho)} .
$$

Also it is worth noting that

$$
W_{0}^{\prime \prime}(\rho)=-2 \frac{\psi^{2}}{(1+\rho)}<0
$$

and so $W_{0}$ is concave.

Consider now the two-period problem. Let the value for the optimizing type in the terminal period be

$$
\begin{aligned}
V(\rho) & =-\frac{1}{2}\left[\left(\psi+x(\rho)-\pi^{*}\right)^{2}+\left(\pi^{*}(x(\rho))\right)^{2}\right] \\
& =-\left(\frac{\psi}{(1+\rho)}\right)^{2}
\end{aligned}
$$


Lets consider the pooling case first. The optimal rule solves

$$
W^{\text {pool }}(\rho)=\max _{\pi, x}-\frac{1}{2}\left[(\psi+x-\pi)^{2}+\pi^{2}\right]+\beta W_{0}(\rho)
$$

subject to

$$
\begin{gathered}
x=\pi \\
-\frac{1}{2}\left[(\psi+x-\pi)^{2}+\pi^{2}\right]+\beta_{\mathrm{o}} V(\rho) \geqslant-\frac{1}{2}\left[\left(\psi+x-\pi^{*}(x)\right)^{2}+\pi^{*}(x)^{2}\right]+\beta_{\mathrm{o}} \mathrm{V}(0)
\end{gathered}
$$

or

$$
W^{\text {pool }}(\rho)=\max _{\pi}-\frac{1}{2}\left[\psi^{2}+\pi^{2}\right]+\beta W_{0}(\rho)
$$

subject to

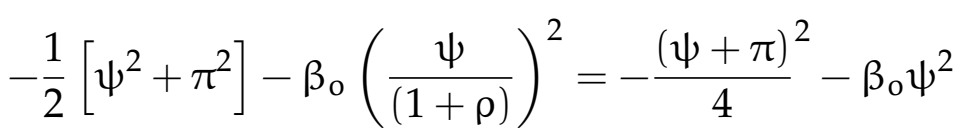

Thus the solution is pinned down by the last constraint. Solving for $\pi$ we have

$$
\pi_{\text {ico }}(\rho)=\psi\left(1-\sqrt{4 \beta_{\mathrm{o}}\left[1-\left(\frac{1}{(1+\rho)}\right)^{2}\right]}\right)
$$

(Note that $\pi_{\text {ico }}(0)=\psi=\pi_{\mathrm{o}}(0)$ and $\pi_{\text {ico }}(1)=\psi\left(1-\sqrt{3 \beta_{\mathrm{o}}}\right)$ so it must be that $\beta_{\mathrm{o}}<1 / 3$.) So the payoff from pooling is

$$
W^{\text {pool }}(\rho)=-\psi^{2}\left[\frac{1}{2}\left[1+\left(1-\sqrt{4 \beta_{\mathrm{o}}\left[1-\left(\frac{1}{(1+\rho)}\right)^{2}\right]}\right)^{2}\right]+\beta \frac{1}{(1+\rho)}\right]
$$

The value of separation is

$$
\begin{aligned}
W^{\text {sep }}(\rho) & =W_{0}(\rho)+\beta\left[\rho W_{0}(1)+(1-\rho) W_{0}(0)\right] \\
& =-\psi^{2}\left[\frac{1}{(1+\rho)}+\beta\left[1-\frac{\rho}{2}\right]\right]
\end{aligned}
$$


Let's consider

$$
\begin{aligned}
& \Delta(\rho) \equiv W^{\text {pool }}(\rho)-W^{\text {sep }}(\rho) \\
&=\psi^{2}\left(-\left[\frac{1}{2}\left[1+\left(1-\sqrt{4 \beta_{\mathrm{o}}\left[1-\left(\frac{1}{(1+\rho)}\right)^{2}\right]}\right)^{2}\right]+\beta \frac{1}{(1+\rho)}\right]\right. \\
&\left.+\left[\frac{1}{(1+\rho)}+\beta\left[1-\frac{\rho}{2}\right]\right]\right) \\
&=\psi^{2}\left(\left[\frac{1}{(1+\rho)}-\frac{1}{2}[1+z(\rho)]\right]+\beta\left[1-\frac{\rho}{2}-\frac{1}{1+\rho}\right]\right)
\end{aligned}
$$

where

$$
z(\rho) \equiv\left(1-\sqrt{4 \beta_{\mathrm{o}}\left[1-\left(\frac{1}{(1+\rho)}\right)^{2}\right]}\right)^{2}=\left(1-\frac{2 \xi}{(1+\rho)} \sqrt{\left[(1+\rho)^{2}-1\right]}\right)^{2}<1
$$

and

$$
\xi \equiv \sqrt{\beta_{\mathrm{o}}}
$$

At $\rho=0, \Delta(0)=0$, while at $\rho=1$,

$$
\Delta(1)=\psi^{2}\left(\left[-\frac{1}{2}\left(1-\sqrt{4 \beta_{\mathrm{o}}\left[1-\left(\frac{1}{2}\right)^{2}\right]}\right)^{2}\right]\right)<0 .
$$

Next, let's look at the slope

$$
\begin{gathered}
\Delta^{\prime}(\rho)=\psi^{2}\left(\left[-\frac{1}{(1+\rho)^{2}}-\frac{1}{2} z^{\prime}(\rho)\right]+\beta\left[-\frac{1}{2}+\frac{1}{(1+\rho)^{2}}\right]\right) \\
z^{\prime}(\rho)=-\left(8 \beta_{0} \frac{1}{(1+\rho)^{3}}\right)\left(\left(4 \beta_{0}\left[1-\left(\frac{1}{(1+\rho)}\right)^{2}\right]\right)^{-\frac{1}{2}}-1\right)
\end{gathered}
$$

Notice that as $\rho \rightarrow 0, z^{\prime}(\rho)$ goes to $-\infty$. Therefore the slope of $\Delta(\rho)$ at $\rho=0$ is

$$
\psi^{2}\left(\left[-1-\frac{1}{2} z^{\prime}(\rho)\right]+\frac{\beta}{2}\right) \rightarrow \infty
$$

so that near 0 there exists a region of pooling. 
In general to get pooling we need

$$
\beta>\frac{\left[-\frac{1}{(1+\rho)}+\frac{1}{2}[1+z(\rho)]\right]}{\left[1-\frac{\rho}{2}-\frac{1}{1+\rho}\right]}
$$

Let

$$
F(\rho) \equiv \frac{\left[-\frac{1}{(1+\rho)}+\frac{1}{2}[1+z(\rho)]\right]}{\left[1-\frac{\rho}{2}-\frac{1}{1+\rho}\right]}
$$

We know that $\lim _{\rho \rightarrow 0} F(\rho)=-\infty$ and $\lim _{\rho \rightarrow 1} F(\rho)=\infty$. In fact, we have

$$
\mathrm{F}(0)=\frac{0}{0}
$$

So by the L'Hôpital's rule we have

$$
\lim _{\rho \rightarrow 0} F(0)=\lim _{\rho \rightarrow 0} \frac{\left[1+\frac{1}{2} z^{\prime}(0)\right]}{\frac{1}{2}}=-\infty
$$

and

$$
\mathrm{F}(1)=\frac{\left[-\frac{1}{2}+\frac{1}{2}[1+z(1)]\right]}{\left[\frac{1}{2}-\frac{1}{2}\right]}=\infty
$$

We next show that $F(\rho)$ is monotone increasing in $\rho$ so that there exists a cutoff $\rho^{*}$ such that it is optimal to pool for $\rho<\rho^{*}$ and it is optimal to separate for $\rho>\rho^{*}$. To this end, note that we can rearrange (42) as

$$
F(\rho)=\frac{-2+(1+\rho)[1+z(\rho)]}{\rho(1-\rho)}
$$

So

$$
F^{\prime}(\rho)=\frac{\rho(1-\rho)\left[(1+\rho) z^{\prime}(\rho)+1+z(\rho)\right]-[-2+(1+\rho)[1+z(\rho)]][1-2 \rho]}{\rho^{2}(1-\rho)^{2}} .
$$

The denominator is positive and so we just need to sign the numerator in order to sign $F^{\prime}(\rho)$.

Lets do some preliminary calculations. We have that

$$
\sqrt{z(\rho)}=1-2 \xi \frac{\sqrt{\left[(1+\rho)^{2}-1\right]}}{(1+\rho)}
$$


and so

$$
\frac{2 \xi}{(1+\rho)}=\frac{1-\sqrt{z(\rho)}}{\sqrt{\left[(1+\rho)^{2}-1\right]}}
$$

Therefore, after some algebraic manipulations, we can rewrite (41) as

$$
\begin{aligned}
z^{\prime}(\rho) & =-\left(8 \beta_{0} \frac{1}{(1+\rho)^{3}}\right)\left(\left(4 \beta_{0}\left[1-\left(\frac{1}{(1+\rho)}\right)^{2}\right]\right)^{-\frac{1}{2}}-1\right) \\
& =-\left(\frac{2}{(1+\rho)}\left(\frac{2 \xi}{(1+\rho)}\right)^{2}\right)\left(\frac{1}{2 \xi \frac{\sqrt{\left[(1+\rho)^{2}-1\right]}}{(1+\rho)}}-1\right) \\
& =-\left(\frac{2}{(1+\rho)} \frac{(1-\sqrt{z(\rho)})^{2}}{\left[(1+\rho)^{2}-1\right]}\right) \frac{\sqrt{z(\rho)}}{1-\sqrt{z(\rho)}} \\
& =-\frac{2}{(1+\rho)} \frac{(\sqrt{z(\rho)}-z(\rho))}{\rho(2+\rho)}
\end{aligned}
$$

where in the third line we used (44).

Next let's consider the numerator of $F^{\prime}(\rho)$ in (43):

$$
\begin{aligned}
h(\rho) & \equiv \rho(1-\rho)\left[(1+\rho) z^{\prime}(\rho)+1+z(\rho)\right]-[-2+(1+\rho)[1+z(\rho)]][1-2 \rho] \\
& =(1-\rho)\left[\frac{-2 \sqrt{z(\rho)}+(2+\rho(2+\rho)) z(\rho)+\rho(2+\rho)}{(2+\rho)}\right] \\
& -\{-2[1-2 \rho]+(1+\rho)[1-2 \rho]+(1+\rho)[1-2 \rho] z(\rho)\} \\
& =\left[\frac{-2(1-\rho) \sqrt{z(\rho)}+\left[\rho+4 \rho^{2}+\rho^{3}\right] z(\rho)+(2+\rho)(1-\rho)^{2}}{(2+\rho)}\right] \\
& >\left[\frac{-2(1-\rho) \sqrt{z(\rho)}+\left[\rho+4 \rho^{2}\right] z(\rho)+(2+\rho)(1-\rho)^{2}}{(2+\rho)}\right] \\
& =\left[\frac{(1-\rho)[(2+\rho)(1-\rho)-2 \sqrt{z(\rho)}]+\left[\rho+4 \rho^{2}\right] z(\rho)}{(2+\rho)}\right]
\end{aligned}
$$

where to obtain the second equality we used (45), the third equality follows from algebra, the inequality follows from $\rho^{3} z(\rho)>0$, and the last equality also follows from algebra. Notice that if $[(2+\rho)(1-\rho)-2 \sqrt{z(\rho)}] \geqslant 0$ then $h(\rho)>0$ and the result is proved. 
Suppose not, i.e.

$$
(1-\rho)<\frac{2 \sqrt{z(\rho)}}{(2+\rho)}
$$

We can then write

$$
\begin{aligned}
h(\rho) & >\left[\frac{(1-\rho)[(2+\rho)(1-\rho)-2 \sqrt{z(\rho)}]+\left[\rho+4 \rho^{2}\right] z(\rho)}{(2+\rho)}\right] \\
& >\left[\frac{\frac{2 \sqrt{z(\rho)}}{(2+\rho)}[(2+\rho)(1-\rho)-2 \sqrt{z(\rho)}]+\left[\rho+4 \rho^{2}\right] z(\rho)}{(2+\rho)}\right] \\
& =\left[\frac{2(1-\rho) \sqrt{z(\rho)}-z(\rho)\left[\frac{4}{(2+\rho)}-\rho-4 \rho^{2}\right]}{(2+\rho)}\right] \\
& >\frac{z(\rho)}{(2+\rho)}\left[2(1-\rho)-\left[\frac{4}{(2+\rho)}-\rho-4 \rho^{2}\right]\right]
\end{aligned}
$$

where the second line follows from (46), the third is algebra, and the fourth line follows from $\sqrt{z(\rho)}>z(\rho)$ since $z(\rho)<1$. We next show that $2(1-\rho)>\left[\frac{4}{(2+\rho)}-\rho-4 \rho^{2}\right]$. To see this, suppose it is not true. Then

$$
2(1-\rho)-\left[\frac{4}{(2+\rho)}-\rho-4 \rho^{2}\right]<0
$$

or

$$
4 \rho^{2}(2+\rho)-\rho^{2}<0
$$

which is a contradiction. Therefore $h(\rho)>0$ and so $F^{\prime}(\rho)>0$. Q.E.D.

\section{A.5 Assumption 4 satisfied in our examples}

Lemma 6. In our two examples, the gains from going to best response are decreasing in $x$. In general, this is true if $\frac{w_{x}\left(\phi(\pi), \pi^{*}(\phi(\pi))\right)}{w_{x}(\phi(\pi), \pi)}$ is close enough to one. 
Proof. In the Barro-Gordon model:

$$
\begin{aligned}
G(x) & =-\frac{1}{2}\left[\left(\psi+x-\frac{\psi+x}{2}\right)^{2}+\left(\frac{\psi+x}{2}\right)^{2}\right]+\frac{1}{2}\left[\psi^{2}+x^{2}\right] \\
& =-\left(\frac{\psi+x}{2}\right)^{2}+\frac{1}{2}\left[\psi^{2}+x^{2}\right] \\
& =-\frac{1}{4}\left[\psi^{2}+x^{2}+2 \psi x\right]+\frac{1}{2}\left[\psi^{2}+x^{2}\right] \\
& =\frac{1}{4}\left[\psi^{2}+x^{2}\right]-\frac{1}{2} \psi x
\end{aligned}
$$

so

$$
\mathrm{G}^{\prime}(x)=\frac{1}{2}(x-\psi)=-\frac{1}{2}(\psi-x)
$$

which is negative for all $x \in[0, \psi]$ i.e. between the Ramsey and the Markov outcome (which is the relevant range).

For the the bailout example, it is more convenient to consider

$$
\tilde{\mathrm{G}}(\pi) \equiv \mathrm{G}(\phi(\pi)) .
$$

Since $\phi$ is strictly decreasing, we have that $\tilde{G}^{\prime}(\pi)=G^{\prime}(\phi(\pi)) \phi^{\prime}(\pi)$ or $G^{\prime}(x)=\tilde{G}^{\prime}\left(\phi^{-1}(x)\right) / \phi^{\prime}(\pi)$ so $\mathrm{G}^{\prime} \leqslant 0$ if $\tilde{\mathrm{G}} \leqslant 0$. Note that

$\tilde{\mathrm{G}}(\pi)=-(1-\mathrm{p}(\phi(\pi)))\left(1-\pi^{*}(\phi(\pi))\right) \psi-\mathrm{c}\left(\pi^{*}(\phi(\pi))\right)+(1-\mathrm{p}(\phi(\pi)))(1-\pi) \psi+\mathrm{c}(\pi)$

Since $(1-p(\phi(\pi))) \psi=c^{\prime}\left(\pi^{*}(\phi(\pi))\right)$ we can write

$$
\begin{aligned}
\tilde{\mathrm{G}}^{\prime}(\pi) & =\mathrm{p}^{\prime}(\phi(\pi)) \phi^{\prime}(\pi)\left(1-\pi^{*}(\phi(\pi))\right) \psi-\mathrm{p}^{\prime}(\phi(\pi)) \phi^{\prime}(\pi)(1-\pi) \psi-(1-\mathrm{p}(\phi(\pi))) \psi+\mathrm{c}^{\prime}(\pi) \\
& =\mathrm{p}^{\prime}(\phi(\pi)) \phi^{\prime}(\pi)\left(\pi-\pi^{*}(\phi(\pi))\right) \psi-\left[(1-\mathrm{p}(\phi(\pi))) \psi-\mathrm{c}^{\prime}(\pi)\right]
\end{aligned}
$$

Recall that

$$
\pi^{*}(e)=\frac{(1-p(e)) \psi}{\lambda}
$$

Thus,

$$
\begin{aligned}
\tilde{\mathrm{G}}(\pi) & =p^{\prime}(\phi(\pi)) \phi^{\prime}(\pi)\left(\pi-\frac{(1-p(\phi(\pi))) \psi}{\lambda}\right) \psi-[(1-p(\phi(\pi))) \psi-\lambda \pi] \\
& =\left[\frac{(1-p(\phi(\pi))) \psi}{\lambda}-\pi\right]\left[-p^{\prime}(\phi(\pi)) \phi^{\prime}(\pi)-\frac{\lambda}{\psi}\right] \psi
\end{aligned}
$$

We are now going to show that the first term in square brackets is positive while the second is negative. Let's start with the first. Since we considering $\pi \in\left[0, \pi_{M}\right]$ it must be 
that for any $\psi, \lambda$, and interior $\pi$, the first term is positive since $\pi<\pi^{*}(\phi(\pi))$. Consider next the second term, $\left[-p^{\prime}(\phi(\pi)) \phi^{\prime}(\pi)-\lambda / \psi\right]$. Note that $-p^{\prime}(\phi(\pi)) \phi^{\prime}(\pi)>0$ and it is increasing in $\pi$. Moreover, $\pi_{M}(\psi)$ is increasing in $\psi$. These two observations imply that we can find a $\psi$ sufficiently small such that the second term is negative for all $\pi \in$ $\left[0, \pi_{M}(\psi)\right]$. Thus, for $\psi$ small enough, we have that $\tilde{G}^{\prime}(\pi) \leqslant 0$.

In general, we have that

$$
\tilde{\mathrm{G}}^{\prime}(\pi)=\left[\mathcal{w}_{x}\left(\phi(\pi), \pi^{*}(\phi(\pi))\right)-\mathcal{w}_{x}(\phi(\pi), \pi)\right] \phi^{\prime}(\pi)-\mathcal{w}_{\pi}(\phi(\pi), \pi)
$$

which is negative if

$$
\mathcal{w}_{\chi}(\phi(\pi), \pi)\left[\frac{\mathcal{w}_{\chi}\left(\phi(\pi), \pi^{*}(\phi(\pi))\right)}{\mathcal{w}_{\chi}(\phi(\pi), \pi)}-1\right] \phi^{\prime}(\pi)-\mathcal{w}_{\pi}(\phi(\pi), \pi) \leqslant 0
$$

which is true if $\frac{w_{\chi}\left(\phi(\pi), \pi^{*}(\phi(\pi))\right)}{\mathcal{w}_{x}(\phi(\pi), \pi)}$ is close enough to one. Q.E.D.

\section{A.6 Proof of Proposition 2}

The problem for the rule designer for a generic horizon $k$ can be written as

$$
W_{k}(\rho)=\max _{x, \pi_{c}, \sigma} \rho\left[w\left(x, \pi_{c}\right)+\beta W_{k-1}\left(\rho^{\prime}\left(\pi_{c}\right)\right)\right]+(1-\rho)\left[w\left(x, \pi_{0}\right)+\beta W_{k-1}\left(\rho^{\prime}\left(\pi_{0}\right)\right)\right]
$$

subject to the implementability condition,

$$
\chi=\phi\left(\rho \pi_{c}+(1-\rho)\left[\sigma \pi_{c}+(1-\sigma) \pi^{*}(x)\right]\right),
$$

the incentive compatibility constraint for the optimizing type,

$$
w\left(x, \pi_{\mathrm{o}}\right)+\beta \mathrm{V}_{\mathrm{k}-1}\left(\rho^{\prime}\left(\pi_{\mathrm{c}}\right)\right) \geqslant \mathcal{w}\left(x, \pi^{*}(x)\right)+\beta \mathrm{V}_{\mathrm{k}-1}\left(\rho^{\prime}\left(\pi_{\mathrm{c}}\right)\right)
$$

and the law of motion for beliefs (7). The value for the optimizing type for a generic horizon $k$ is

$$
V_{k}(\rho)=w\left(x_{k}(\rho), \pi_{o, k}(\rho)\right)+\beta_{o} V_{k-1}\left(\rho^{\prime}\left(\pi_{o, k}(\rho)\right)\right)
$$

As for the twice repeated case, we can just compare two values:

$$
W_{k}(\rho)=\max \left\{W_{k}^{\text {pool }}(\rho), W_{k}^{\text {sep }}(\rho)\right\}
$$

where

$$
W_{k}^{s e p}(\rho)=W_{0}(\rho)+\beta\left[\rho W_{k-1}(1)+(1-\rho) W_{k-1}(0)\right]
$$




$$
W_{k}^{\text {pool }}(\rho)=w\left(x_{i c o, k}(\rho), \phi^{-1}\left(x_{i c o, k}(\rho)\right)\right)+\beta W_{k-1}(\rho)
$$

and $x_{i c o, k}(\rho)$ solves

$$
\mathrm{G}\left(x_{i c o, k}(\rho)\right)=\beta \Delta V_{k-1}(\rho)
$$

where we define

$$
\Delta V_{k}(\rho) \equiv V_{k}(\rho)-V_{k}(0) .
$$

and recall that $\mathrm{G}(\mathrm{x})=w\left(x, \pi^{*}(\mathrm{x})\right)-w\left(x, \phi^{-1}(\mathrm{x})\right)$.

With this setup, we can now turn to the proof of the proposition (as usual we consider the case with $w_{x}<0$ ):

Part 1. For $\rho=0$ it is clear that the equilibrium is the repetition of the static outcome i.e. all $k, W_{k}(0)=W_{0} /(1-\beta)$ and $V_{k}(0)=V_{0}(0) /\left(1-\beta_{0}\right)$, since no incentives can be provided to the optimizing type.

Part 2. Consider now the region $\rho \in\left(0, \rho^{*}\right]$. We will show that $\left.i\right)$ in this region it is always optimal to pool and ii) the optimal rule converges monotonically to $\pi_{\mathrm{CK}}$. We will do so by an induction argument. Before doing so, we prove an intermediate result: $\rho^{*}<\rho_{1}^{*}$.

To prove this we need to show that at $\rho^{*}$, with residual horizon of 1 (twice repeated) the rule designer strictly prefers to pool, i.e.,

$$
w\left(x_{\text {ico }, 1}\left(\rho^{*}\right), \pi_{\text {ico }, 1}\left(\rho^{*}\right)\right)+\beta W_{0}\left(\rho^{*}\right) \geqslant W_{0}\left(\rho^{*}\right)+\beta\left[\rho^{*} W_{0}(1)+\left(1-\rho^{*}\right) W_{0}(0)\right]
$$

while

$$
w\left(x_{C K}, \pi_{C K}\right)+\frac{\beta}{1-\beta} w\left(x_{C K}, \pi_{C K}\right)=W_{0}\left(\rho^{*}\right)+\frac{\beta}{1-\beta}\left[\rho^{*} W_{0}(1)+\left(1-\rho^{*}\right) W_{0}(0)\right]
$$

To show this, it is sufficient to show that at $\rho^{*}$, both the static payoff and the continuation value of pooling are larger with horizon 1 than for the infinitely repeated economy, i.e.

$$
w\left(x_{\text {ico, } 1}\left(\rho^{*}\right), \pi_{\text {ico, } 1}\left(\rho^{*}\right)\right)>w\left(x_{\mathrm{CK}}, \pi_{\mathrm{CK}}\right)
$$

and

$\beta\left[W_{0}\left(\rho^{*}\right)-\left[\rho W_{0}(1)+(1-\rho) W_{0}(0)\right]\right]>\frac{\beta}{1-\beta}\left[w\left(x_{C K}, \pi_{C K}\right)-\left[\rho W_{0}(1)+(1-\rho) W_{0}(0)\right]\right]$

Let's first compare the static values. To prove condition (53), it is sufficient to show that $\pi_{\mathrm{ico}, 1}\left(\rho^{*}\right)<\pi_{\mathrm{CK}}$ or equivalently $x_{\mathrm{ico}, 1}\left(\rho^{*}\right)<x_{\mathrm{CK}}$ since $w_{\mathrm{x}}<0$. To this end, we first 
show that $x_{0}\left(\rho^{*}\right)<x_{C K}$. Suppose not, i.e. $x_{C K} \leqslant x_{0}\left(\rho^{*}\right)$. Then

$$
\begin{aligned}
W_{0}\left(\rho^{*}\right) & =\rho w\left(x_{0}\left(\rho^{*}\right), \underline{\pi}\right)+\left(1-\rho^{*}\right) w\left(x_{0}\left(\rho^{*}\right), \pi^{*}\left(x_{0}\left(\rho^{*}\right)\right)\right) \\
& <w\left(x_{0}\left(\rho^{*}\right), \rho^{*} \underline{\pi}+\left(1-\rho^{*}\right) \pi^{*}\left(x_{0}\left(\rho^{*}\right)\right)\right) \\
& =w\left(x_{0}\left(\rho^{*}\right), \phi^{-1}\left(x_{0}\left(\rho^{*}\right)\right)\right) \\
& \leqslant w\left(x_{C K}, \pi_{C K}\right)
\end{aligned}
$$

where the first line is the definition of $W_{0}(\rho)$, the second line follows from the concavity of $w$, the third line is algebra, and the fourth line follows from the contradiction hypothesis $x_{C K} \leqslant x_{0}\left(\rho^{*}\right)$. We next show that this is a contradiction because the definition of $\rho^{*}$ implies that there must be static losses associated with pooling, i.e. $W_{0}\left(\rho^{*}\right)>w\left(x_{C K}, \pi_{C K}\right)$. Suppose not, i.e. $W_{0}\left(\rho^{*}\right) \leqslant w\left(x_{\mathrm{CK}}, \pi_{\mathrm{CK}}\right)$ then

$$
\frac{w\left(x_{\mathrm{CK}}, \pi_{\mathrm{CK}}\right)}{1-\beta} \geqslant \frac{W_{0}\left(\rho^{*}\right)}{1-\beta}>W_{0}\left(\rho^{*}\right)+\frac{\beta}{1-\beta}\left[\rho^{*} W_{0}(1)+\left(1-\rho^{*}\right) W_{0}(0)\right]
$$

which contradicts (52). Thus it must be that $x_{C K} \leqslant x_{0}\left(\rho^{*}\right)$.

We now turn to show that $x_{i c o, 1}\left(\rho^{*}\right)<x_{C K}$. Let's consider the incentive constraint for the optimizing type in the twice repeated economy:

$$
w\left(x_{i c o, 1}\left(\rho^{*}\right), \phi^{-1}\left(\pi_{\text {ico }, 1}\left(\rho^{*}\right)\right)\right)+\beta V_{0}\left(\rho^{*}\right)=w\left(x_{i c o, 1}\left(\rho^{*}\right), \pi^{*}\left(x_{i c o, 1}\left(\rho^{*}\right)\right)\right)+\beta V_{0}(0)
$$

and in the infinite horizon economy, (9), reported here for convenience,

$$
\mathrm{V}_{\mathrm{CK}} \equiv \frac{w\left(\mathrm{x}_{\mathrm{CK}}, \phi^{-1}\left(\mathrm{x}_{\mathrm{CK}}\right)\right)}{1-\beta}=w\left(\mathrm{x}_{\mathrm{CK}}, \pi^{*}\left(\mathrm{x}_{\mathrm{CK}}\right)\right)+\frac{\beta_{\mathrm{o}}}{1-\beta_{\mathrm{O}}} \mathrm{V}_{0}(0)
$$

Recall that

$$
\mathrm{G}(\mathrm{x})=w\left(x, \pi^{*}(x)\right)-w\left(x, \phi^{-1}(x)\right)
$$

is decreasing in $x$. Also note that by the definition of $\rho^{*}$,

$$
\mathrm{G}\left(\mathrm{x}_{\mathrm{CK}}\right)=\Delta \mathrm{V}_{\mathrm{CK}}\left(\rho^{*}\right)
$$

where

$$
\Delta \mathrm{V}_{\mathrm{CK}}(\rho) \equiv \mathrm{V}_{\mathrm{CK}}-\frac{\mathrm{V}_{0}(0)}{1-\beta_{\mathrm{O}}} .
$$

Therefore, if $\Delta V_{0}\left(\rho^{*}\right)>\Delta V_{C K}$, then $x_{i c o, 1}\left(\rho^{*}\right)<x_{C K}$. We now show that $\Delta V_{0}\left(\rho^{*}\right)>\Delta V_{C K}$. 
Define

$$
\begin{aligned}
\mathrm{V}_{\mathrm{CK}}^{\text {sep }}\left(\rho^{*}\right) & \equiv w\left(x_{0}\left(\rho^{*}\right), \pi^{*}\left(x_{0}\left(\rho^{*}\right)\right)\right)+\frac{\beta_{\mathrm{O}}}{1-\beta_{\mathrm{O}}} \mathrm{V}_{0}(0) \\
& >w\left(x_{\mathrm{CK}}, \pi^{*}\left(x_{\mathrm{CK}}\right)\right)+\frac{\beta_{\mathrm{O}}}{1-\beta_{\mathrm{O}}} \mathrm{V}_{0}(0) \\
& =w\left(x_{\mathrm{CK}}, \phi^{-1}\left(x_{\mathrm{CK}}\right)\right)+\beta_{\mathrm{O}} \mathrm{V}_{\mathrm{CK}} \\
& =\mathrm{V}_{\mathrm{CK}}
\end{aligned}
$$

where the second line follows from $x_{C K}>x_{0}\left(\rho^{*}\right)$ and $w_{x}<0$, the third line from the incentive compatibility constraint (55), and the last line from the definition of $\mathrm{V}_{\mathrm{CK}}$. Thus,

$$
\begin{aligned}
\Delta V_{C K} & =V_{C K}-\frac{V_{0}(0)}{1-\beta_{o}} \\
& <V_{C K}^{\text {sep }}\left(\rho^{*}\right)-\frac{V_{0}(0)}{1-\beta_{o}} \\
& =V_{0}\left(\rho^{*}\right)+\frac{\beta_{o}}{1-\beta_{o}} V_{0}(0)-\frac{V_{0}(0)}{1-\beta_{o}} \\
& =V_{0}\left(\rho^{*}\right)-V_{0}(0)=\Delta V_{0}\left(\rho^{*}\right)
\end{aligned}
$$

where the second line follows from (56), the third from the definition of $V_{C K}^{\text {sep }}(\rho)$, and the last line follows from algebra. Thus $\Delta V_{C K}<\Delta V_{0}\left(\rho^{*}\right)$ and consequently $\pi_{\text {ico, } 1}\left(\rho^{*}\right)<\pi_{C K}$. Thus condition (53) holds.

We now compare the continuation values and show that (54) holds. Note from the definition of $\rho^{*},(52)$, it follows that

$$
\begin{aligned}
& W_{0}\left(\rho^{*}\right)=\frac{w\left(x_{C K}, \pi_{C K}\right)}{1-\beta}-\frac{\beta}{1-\beta}\left[\rho W_{0}(1)+(1-\rho) W_{0}(0)\right] \\
\Longrightarrow & W_{0}\left(\rho^{*}\right)-\left[\rho W_{0}(1)+(1-\rho) W_{0}(0)\right]=\frac{1}{1-\beta}\left[w\left(x_{C K}, \pi_{C K}\right)-\left[\rho W_{0}(1)+(1-\rho) W_{0}(0)\right]\right] .
\end{aligned}
$$

Therefore, the dynamic benefits are identical and condition (54) holds as an equality. Thus, $\rho_{1}^{*}>\rho^{*}$.

We are now ready to consider the main induction argument. Define

$$
\begin{aligned}
& W_{k+1}^{\text {pool }}(\rho) \equiv \sum_{j=0}^{k} \beta^{j} w\left(x_{k+1-j}(\rho), \phi^{-1}\left(x_{k+1-j}(\rho)\right)\right)+\beta^{k+1} W_{0}(\rho) \\
& W_{k+1}^{\text {sep }}(\rho) \equiv W_{0}(\rho)+\sum_{j=1}^{k+1} \beta^{j}\left[\rho W_{0}(1)+(1-\rho) W_{0}(0)\right]
\end{aligned}
$$


where $x_{k}$ is short-hand notation for $x_{i c o, k}$.

We will show that for $\rho \in\left(0, \rho^{*}\right]$, if i) $W_{j}^{\text {pool }}(\rho) \geqslant W_{j}^{\text {sep }}(\rho)$ for all $j \leqslant k$ and ii) $\left\{x_{j}(\rho)\right\}_{j=1}^{k}$ is monotone then i) $W_{k+1}^{\text {pool }}(\rho) \geqslant W_{k+1}^{\text {sep }}(\rho)$ and ii) $\left\{x_{j}(\rho)\right\}_{j=1}^{k+1}$ is monotone.

We will use the following observations: From (51), $x_{k}$ is such that

$$
G\left(x_{k}(\rho)\right)=\beta_{0} \Delta V_{k-1}(\rho)
$$

Moreover, for any $k \geqslant 1$

$$
\begin{aligned}
\mathrm{V}_{k}^{\text {pool }}(\rho) & =\sum_{j=0}^{k} \beta_{\mathrm{o}}^{j} w\left(x_{k+1-j}(\rho), \phi^{-1}\left(x_{k+1-j}(\rho)\right)\right)+\beta_{o}^{k+1} V_{0}(\rho) \\
& =w\left(x_{k}(\rho), \phi^{-1}\left(x_{k}(\rho)\right)\right)+\beta_{o} V_{k-1}^{\text {pool }}(\rho)
\end{aligned}
$$

Thus if it is optimal to pool for all $k, k-1, \ldots, 1$, the dynamic incentives are given by

$$
\begin{aligned}
\Delta \mathrm{V}_{\mathrm{k}}(\rho) & =\mathrm{V}_{\mathrm{k}}^{\text {pool }}(\rho)-\mathrm{V}_{\mathrm{k}}(0) \\
& =w\left(x_{k}(\rho), \phi^{-1}\left(x_{\mathrm{k}}(\rho)\right)\right)+\beta_{\mathrm{o}} \mathrm{V}_{\mathrm{k}-1}^{\text {pool }}(\rho)-\mathrm{V}_{\mathrm{k}}(0) \\
& =w\left(x_{k}(\rho), \pi^{*}\left(\mathrm{x}_{\mathrm{k}}(\rho)\right)\right)+\beta_{\mathrm{o}} \mathrm{V}_{\mathrm{k}-1}(0)-\mathrm{V}_{\mathrm{k}}(0) \\
& =w\left(x_{k}(\rho), \pi^{*}\left(x_{k}(\rho)\right)\right)-\mathrm{V}_{0}(0)
\end{aligned}
$$

where the third line follows from (51) and the last from $V_{k}(0)=\left(1+\beta_{o}+\beta_{o}^{2}+\ldots+\beta_{o}^{k}\right) V_{0}(0)$. Let's define

$$
\mathrm{F}(\mathrm{x}) \equiv \mathcal{w}\left(\mathrm{x}, \pi^{*}(\mathrm{x})\right)-\mathrm{V}_{0}(0) .
$$

Clearly $F(x)$ is a strictly decreasing function.Combining (57) and (58) using the definition of $F$, we have that for all $k \geqslant 1$

$$
F\left(x_{k}(\rho)\right)=\Delta V_{k}(\rho)=\frac{1}{\beta_{o}} G\left(x_{k+1}(\rho)\right) .
$$

We can now proceed with the induction argument.

For $k=1$ : Since $\rho^{*}<\rho_{1}^{*}$, it is optimal to pool and so $W_{1}^{\text {pool }}(\rho)>W_{1}^{\text {sep }}(\rho)$ for all $\rho \in\left(0, \rho^{*}\right]$.

Consider now a generic horizon $k+1$. Suppose that $\left\{x_{j}(\rho)\right\}_{j=1}^{k}$ is monotone decreasing. Then $x_{k} \leqslant x_{k-1}$ so using (59) and the fact that $G$ and $F$ are strictly decreasing functions we have

$$
\frac{1}{\beta_{\mathrm{o}}} \mathrm{G}\left(\mathrm{x}_{\mathrm{k}+1}\right)=\mathrm{F}\left(\mathrm{x}_{\mathrm{k}}\right) \geqslant \mathrm{F}\left(\mathrm{x}_{\mathrm{k}-1}\right)=\frac{1}{\beta_{\mathrm{o}}} \mathrm{G}\left(\mathrm{x}_{\mathrm{k}}\right)
$$


Since $\mathrm{G}$ is strictly decreasing, the above implies that

$$
x_{k+1} \leqslant x_{k} \leqslant x_{k-1}
$$

so $\left\{x_{j}(\rho)\right\}_{j=1}^{k+1}$ is monotone decreasing. A symmetric argument can be made if $\left\{x_{j}(\rho)\right\}_{j=1}^{k}$ is monotone increasing.

Next we show that it is optimal to pool in $k+1$. Note that

$$
\begin{aligned}
W_{k+1}^{\text {pool }}(\rho)-W_{k+1}^{\text {sep }}(\rho) & =w\left(x_{k+1}, \phi^{-1}\left(x_{k+1}\right)\right)+\beta W_{k}^{\text {pool }}(\rho) \\
& -\left\{W_{0}(\rho)+\frac{\beta\left(1-\beta^{k}\right)}{1-\beta}\left[\rho W_{0}(1)+(1-\rho) W_{0}(0)\right]\right\} \\
& \geqslant w\left(x_{k+1}, \phi^{-1}\left(x_{k+1}\right)\right)+\beta\left\{W_{0}(\rho)+\frac{\beta\left(1-\beta^{k-1}\right)}{1-\beta}\left[\rho W_{0}(1)+(1-\rho) W_{0}(0)\right]\right\} \\
& -\left\{W_{0}(\rho)+\frac{\beta\left(1-\beta^{k}\right)}{1-\beta}\left[\rho W_{0}(1)+(1-\rho) W_{0}(0)\right]\right\} \\
& =w\left(x_{k+1}, \phi^{-1}\left(x_{k+1}\right)\right)-(1-\beta) W_{0}(\rho)+\beta\left[\rho W_{0}(1)+(1-\rho) W_{0}(0)\right] \\
& =w\left(x_{k+1}, \phi^{-1}\left(x_{k+1}\right)\right)-W_{0}(\rho)+\beta\left[W_{0}(\rho)-\rho W_{0}(1)+(1-\rho) W_{0}(0)\right]
\end{aligned}
$$

where the first equality just uses the definitions of $W_{k+1}^{\text {pool }}$ and $W_{k+1}^{\text {sep }}$, the second inequality follows from $W_{k}^{\text {pool }} \geqslant W_{k}^{\text {sep }}$ which follows from the induction hypothesis, and the final equality follows from algebra. The last term is positive if and only if

$$
\frac{w\left(x_{k+1}, \phi^{-1}\left(x_{k+1}\right)\right)}{1-\beta} \geqslant W_{0}(\rho)+\frac{\beta}{1-\beta}\left[\rho W_{0}(1)+(1-\rho) W_{0}(0)\right]
$$

Suppose first that $\left\{x_{j}(\rho)\right\}_{j=1}^{k}$ is monotone decreasing. Then $x_{k+1} \leqslant x_{k}$ and so

$$
\frac{w\left(x_{k+1}, \phi^{-1}\left(x_{k+1}\right)\right)}{1-\beta} \geqslant \frac{w\left(x_{k}, \phi^{-1}\left(x_{k}\right)\right)}{1-\beta} \geqslant W_{0}(\rho)+\frac{\beta}{1-\beta}\left[\rho W_{0}(1)+(1-\rho) W_{0}(0)\right]
$$

because $w_{x}<0$. Therefore, $W_{k+1}^{\text {pool }}(\rho)-W_{k+1}^{\text {sep }}(\rho)>0$.

If instead $\left\{x_{j}(\rho)\right\}_{j=1}^{k}$ is monotone increasing then $x_{k+1} \geqslant x_{k}$ so we cannot use the above argument. However, (60) holds if $x_{k+1} \leqslant x_{C K}$. Thus we need to show that if $x_{k} \leqslant x_{C K}$ then $x_{k+1} \leqslant x_{C K}$. Notice that since

$$
w\left(x_{C K}, \phi^{-1}\left(x_{C K}\right)\right)=\left(1-\beta_{0}\right) w\left(x_{C K}, \pi^{*}\left(x_{C K}\right)\right)+\beta_{\mathrm{o}} V_{0}(0)
$$


we have that

$$
\mathrm{F}\left(\mathrm{x}_{\mathrm{CK}}\right)=\frac{w\left(\mathrm{x}_{\mathrm{CK}}, \phi^{-1}\left(\mathrm{x}_{\mathrm{CK}}\right)\right)-\mathrm{V}_{0}(0)}{\left(1-\beta_{\mathrm{o}}\right)}
$$

and

$$
\mathrm{G}\left(\mathrm{x}_{\mathrm{CK}}\right)=w\left(\mathrm{x}_{\mathrm{CK}}, \pi^{*}\left(\mathrm{x}_{\mathrm{CK}}\right)\right)-w\left(\mathrm{x}_{\mathrm{CK}}, \phi^{-1}\left(\mathrm{x}_{\mathrm{CK}}\right)\right)=\beta_{\mathrm{o}} \frac{w\left(\mathrm{x}_{\mathrm{CK}}, \phi^{-1}\left(\mathrm{x}_{\mathrm{CK}}\right)\right)-\mathrm{V}_{0}(0)}{\left(1-\beta_{\mathrm{o}}\right)}
$$

so

$$
F\left(x_{\mathrm{CK}}\right)=\frac{1}{\beta_{\mathrm{o}}} \mathrm{G}\left(\mathrm{x}_{\mathrm{CK}}\right)
$$

Since for all $k$,

$$
F\left(x_{k}\right)=\frac{1}{\beta} G\left(x_{k+1}\right)
$$

if $x_{k} \leqslant x_{C K}$ and $F$ and $G$ are strictly decreasing we have that

$$
F\left(x_{k}\right) \geqslant F\left(x_{C K}\right) \Rightarrow G\left(x_{k+1}\right) \geqslant G\left(x_{C K}\right) \Rightarrow x_{k+1} \leqslant x_{C K}
$$

Therefore condition (60) holds and it is optimal to pool when the horizon is $k+1$. This concludes the proof of the induction step.

Summarizing, we have proved that for $\rho \in\left(0, \rho^{*}\right]$, for all $k \geqslant 1$ it is optimal to pool and $\left\{x_{k}(\rho)\right\}_{k \geqslant 1}$ is a monotone and bounded sequence. The fact that the sequence if bounded follows from $x \in\left[\phi(\underline{\pi}), \pi_{0}(0)\right]$. Then $\left\{x_{k}(\rho)\right\}_{k \geqslant 1}$ must converge pointwise to some limit $x(\rho)$. To see that $x(\rho)=x_{C K}$, note that for all $k$

$$
\begin{aligned}
G\left(x_{k}(\rho)\right) & =\beta_{o}\left[V_{k}^{\text {pool }}(\rho)-V_{k}(0)\right] \\
& =\beta_{o}\left[\sum_{j=0}^{k} \beta^{j} w\left(x_{k+1-j}(\rho), \phi^{-1}\left(x_{k+1-j}(\rho)\right)\right)+\beta^{k+1} V_{0}(\rho)-\frac{1-\beta_{o}^{k+1}}{1-\beta_{o}} V_{0}(0)\right]
\end{aligned}
$$

Taking limits as $k \rightarrow \infty$ we have that the limit $\chi(\rho)$ is implicitly defined by

$$
G(x(\rho))=\beta_{\mathrm{o}}\left[\frac{w\left(x(\rho), \phi^{-1}(x(\rho))\right)}{1-\beta_{\mathrm{o}}}-\frac{\mathrm{V}_{0}(0)}{1-\beta_{\mathrm{o}}}\right]
$$

or

$$
\frac{w\left(x(\rho), \phi^{-1}(x(\rho))\right)}{1-\beta_{0}}=w\left(x(\rho), \pi^{*}(x(\rho))\right)+\beta_{0} \frac{V_{0}(0)}{1-\beta_{o}}
$$

which is the implicit definition of $x_{C K}$ (and there is a unique solution).

Part 3. Consider now $\rho \in\left(\rho_{1}^{*}, 1\right]$. Here we know that in the twice repeated problem it is optimal to separate in the first period. We now show it is also optimal to separate for all horizons $k \geqslant 2$. Consider any horizon $k+1$ with $k \geqslant 1$. Suppose it is optimal to separate 
for horizons $0,1, \ldots, k$ at prior $\rho$. We next show it is optimal to separate in $k+1$ Note that regardless of the horizon, if there is separation next period we have that

$$
\begin{aligned}
\Delta V_{k}(\rho) & =\left[V_{0}(\rho)+\left(\beta+\beta^{2}+\ldots+\beta^{k}\right) V_{0}(0)\right]-\left(1+\beta+\beta^{2}+\ldots+\beta^{k}\right) V_{0}(0) \\
& =V_{0}(\rho)-V_{0}(0)
\end{aligned}
$$

Thus $x_{k+1}(\rho)=x_{k}(\rho)$. Therefore

$$
\begin{aligned}
& W_{k+1}^{\text {pool }}(\rho)=W\left(x_{k}(\rho), \phi^{-1}\left(x_{k}(\rho)\right)\right)+\beta W_{0}(\rho)+\left(\beta^{2}+\beta^{3}+\ldots+\beta^{k+1}\right)\left[\rho W_{0}(1)+(1-\rho) W_{0}(0)\right] \\
& W_{k+1}^{\text {sep }}(\rho)=W_{0}(\rho)+\left(\beta+\beta^{2}+\beta^{3}+\ldots+\beta^{k+1}\right)\left[\rho W_{0}(1)+(1-\rho) W_{0}(0)\right]
\end{aligned}
$$

and

$$
\begin{aligned}
W_{k+1}^{\text {sep }}(\rho)-W_{k+1}^{\text {pool }}(\rho) & =\left[W_{0}(\rho)-\mathcal{W}\left(x_{k}(\rho), \phi^{-1}\left(x_{k}(\rho)\right)\right)\right] \\
& -\beta\left\{W_{0}(\rho)-\left[\rho W_{0}(1)+(1-\rho) W_{0}(0)\right]\right\} \\
& =W_{1}^{\text {sep }}(\rho)-W_{1}^{\text {pool }}(\rho)>0
\end{aligned}
$$

Thus for $\rho>\rho_{1}^{*}$ it is always optimal to separate for any horizon.

Part 4. We now show that for $\rho \in\left(\rho^{*}, \rho_{1}^{*}\right)$ the equilibrium rules do not converge as the horizon goes to infinity and they display a cyclical pattern.This is because there is a form a strategic substitutability across time. In particular, if it is optimal to separate with horizon $k$ then it is optimal to pool with residual horizon $k+1$ if $\rho \in\left(\rho^{*}, \rho_{1}^{*}\right)$. To see this, note that since it is optimal to separate with horizon $k$ then the values of pooling and separating with horizon $k+1$ are given by

$$
\begin{aligned}
& W_{k+1}^{\text {pool }}(\rho)=w\left(x_{k+1}\right)+\beta W_{0}(\rho)+\left(\beta^{2}+\ldots+\beta^{k}\right)\left[\rho W_{0}(1)+(1-\rho) W_{0}(0)\right] \\
& W_{k+1}^{\text {sep }}(\rho)=W_{0}(\rho)+\left(\beta+\beta^{2}+\ldots+\beta^{k}\right)\left[\rho W_{0}(1)+(1-\rho) W_{0}(0)\right]
\end{aligned}
$$

so

$$
W_{k+1}^{\text {pool }}(\rho)-W_{k+1}^{\text {sep }}(\rho)=w\left(x_{k+1}\right)+\beta W_{0}(\rho)-W_{0}(\rho)-\beta\left[\rho W_{0}(1)+(1-\rho) W_{0}(0)\right]
$$

The dynamic incentives provided to the optimizing type are

$$
\begin{aligned}
\Delta V_{k}(\rho) & =V_{0}(\rho)+\left(\beta+\ldots+\beta^{k-1}\right) V_{0}(\rho)-\left(1+\beta+\ldots+\beta^{k-1}\right) V_{0}(0) \\
& =V_{0}(\rho)-V_{0}(\rho)=\Delta V_{0}(\rho)
\end{aligned}
$$


so $x_{k+1}=x_{1}$. Thus, from (61) it follows that

$$
W_{k+1}^{\text {pool }}(\rho)-W_{k+1}^{\text {sep }}(\rho)=W_{1}^{\text {pool }}(\rho)-W_{1}^{\text {sep }}(\rho)>0
$$

so it is optimal to pool if the next period (horizon $k$ ) rule designer separates.

Since in the last period, $k=0$, it is optimal to separate, the above logic implies that pooling is optimal at $k=1$. We now argue that the consecutive number of periods of pooling must be finite. Suppose to the contrary that they are not. Then the value of pooling converges to $w\left(x_{C K}, \phi^{-1}\left(x_{C K}\right)\right) /(1-\beta)$ which is less than the value of separating because $\rho>\rho^{*}$. Then the finite consecutive number of periods with pooling, $M(\rho)$, is is the largest natural number such that the value of pooling is greater than the value of separating implicitly defined by

$$
\begin{aligned}
& \sum_{j=1}^{M(\rho)} \beta^{M(\rho)-j} \mathcal{W}\left(x_{j}, \phi^{-1}\left(x_{j}\right)\right)+\beta^{M(\rho)}\left\{W_{0}(\rho)+\frac{\beta}{1-\beta}\left[\rho W_{0}(1)+(1-\rho) W_{0}(0)\right]\right\} \\
& \geqslant W_{0}(\rho)+\frac{\beta}{1-\beta}\left[\rho W_{0}(1)+(1-\rho) W_{0}(0)\right]
\end{aligned}
$$

where the sequence $\left\{x_{j}\right\}_{j=1}^{M(\rho)}$ solves

$$
\begin{aligned}
F\left(x_{0}(\rho)\right) & =\frac{1}{\beta} G\left(x_{1}(\rho)\right) \\
& \vdots= \\
F\left(x_{M-1}(\rho)\right) & =\frac{1}{\beta} G\left(x_{M}(\rho)\right)
\end{aligned}
$$

given $x_{0}(\rho)$ from the static problem.

The optimal rule as a function of the residual horizon is then

$$
\pi_{k}(\rho)= \begin{cases}\underline{\pi} & \text { if } k=0, M(\rho)+1, \ldots, n(M(\rho)+1), \ldots \\ \phi^{-1}\left(x_{1}(\rho)\right) & \text { if } k=1, M(\rho)+2, \ldots, n(M(\rho)+1)+1, \ldots \\ \vdots & \vdots \\ \phi^{-1}\left(x_{M}(\rho)\right) & \text { if } k=M(\rho), 2 M(\rho)+1, \ldots, n(M(\rho)+1)+M(\rho), \ldots\end{cases}
$$

so the the optimal strategy has $M+1$ period-cycles. Q.E.D.

Note that for $\rho \in\left(\rho^{*}, \rho_{1}^{*}\right)$ the equilibrium strategies display a cyclical pattern but not the equilibrium outcomes. This is because the first time the strategy for the rule designer calls for a stringent rule $-\underline{\pi}-$ to induce separation then the posterior converges to either 0 or 1 and these two states are absorbing. However, we could observe equilibrium 
Figure 6: Strategies and outcomes when $\rho \in\left(\rho^{*}, \rho_{1}^{*}\right)$

Equilibrium strategies when $M(\rho)=2$
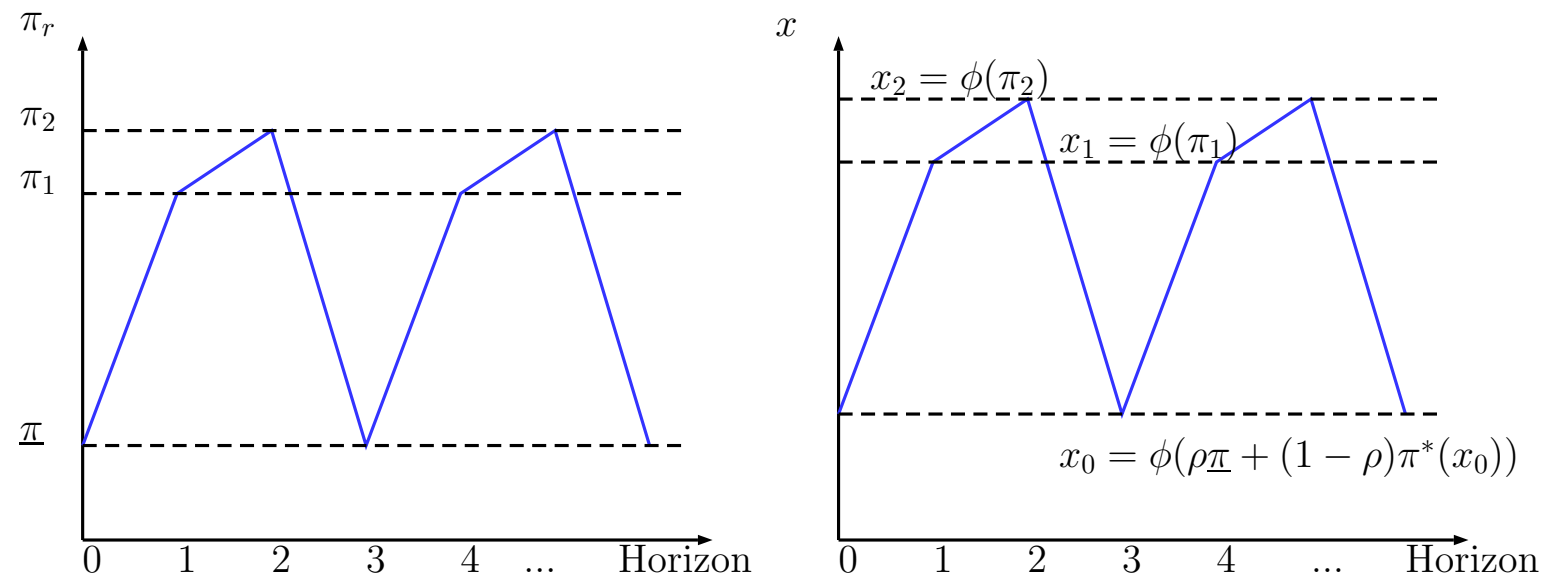

Equilibrium outcome when $M(\rho)=2$ and $K=3$
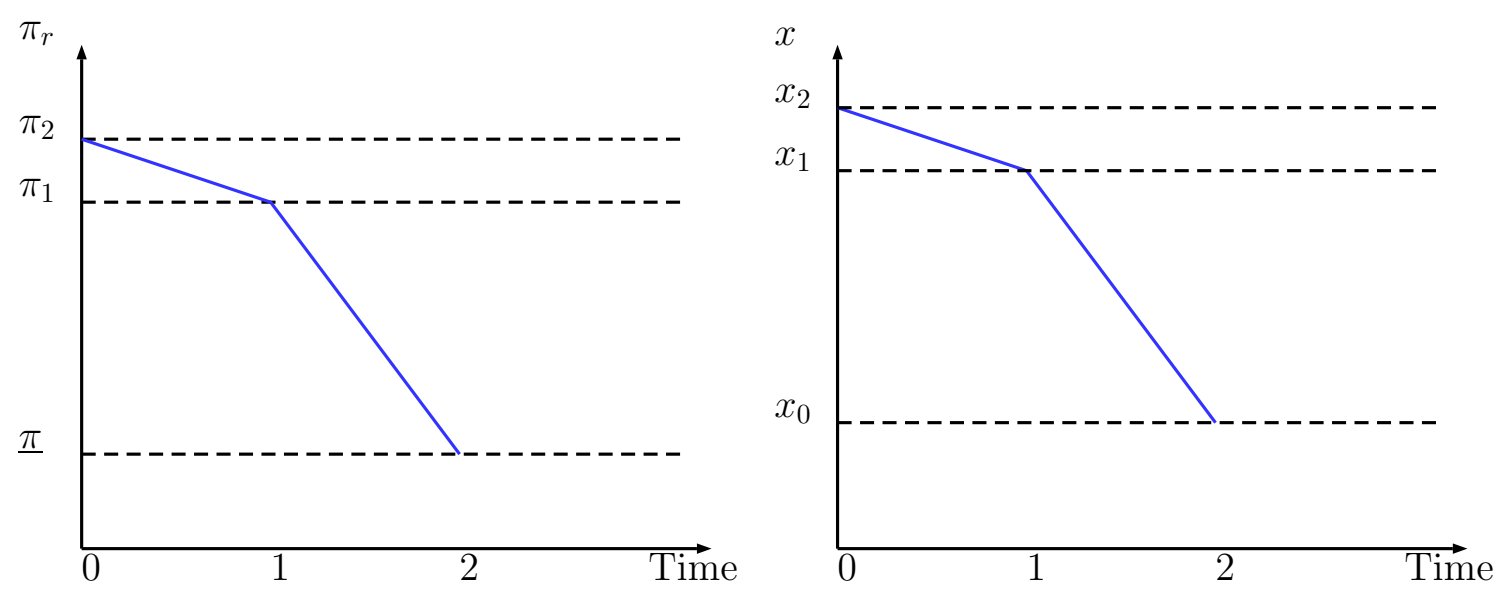

Equilibrium outcome when $M(\rho)=2$ and $K=4$
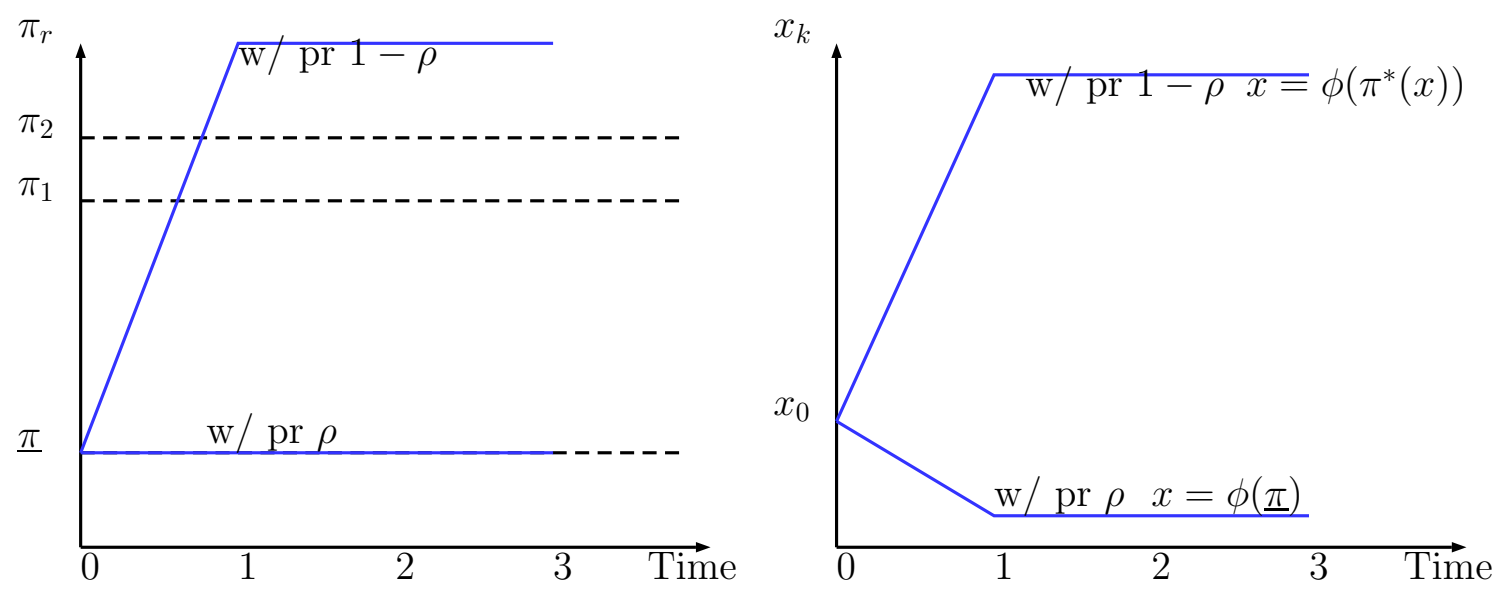
Figure 7: Equilibrium values: uncertainty is beneficial for $k \geqslant 1$

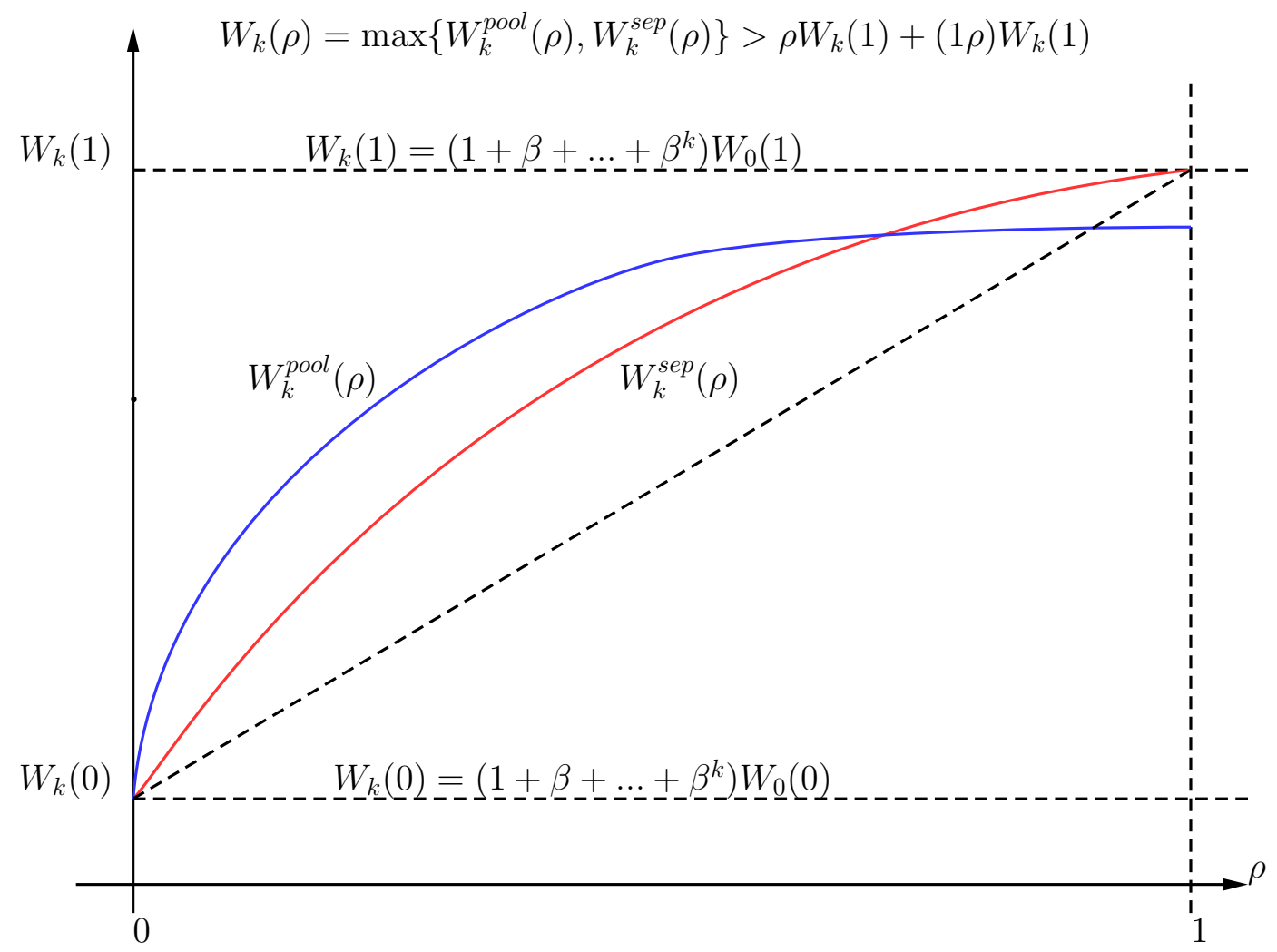

dynamics that depend on horizon K. To see this, consider the following example. For concreteness, suppose $M(\rho)=2$. The equilibrium strategies for the rule designers as a function of $k$ are reported in the first panel of Figure 6. If we start the economy with an horizon of $K=M(\rho)+1=3$, the equilibrium outcome has two periods of pooling with progressively more stringent rules and finally there is separation in the final period when the recommendation is $\underline{\pi}$ as displayed in the second panel of Figure 6 . If instead we start the economy with an horizon of $\mathrm{K}=4$ then it is optimal to separate in the very first period and then the equilibrium outcome is either the Ramsey outcome (with probability $\rho$ ) or the static Markov outcome (with probability $1-\rho$ ). This is illustrated in the third panel of Figure 6. Thus the equilibrium outcome critically depends on the horizon of the economy when $\rho \in\left(\rho^{*}, \rho_{1}^{*}\right)$.

Moreover, note that if $W_{0}(\rho)$ is concave then uncertainty is beneficial at any horizon $k \geqslant 0$, in that $W_{k}(\rho)>\rho W_{k}(1)+(1-\rho) W_{k}(0)$ for all $k$ as shown in Figure 7. This is true despite the fact that for a general horizon $k, W_{k}(\rho)$ is the upper envelope of two (concave) functions, $W_{k}(\rho)=\max \left\{W_{k}^{\text {pool }}(\rho), W_{k}^{\text {sep }}(\rho)\right\}$, and therefore it is not globally concave.

Lemma 7. If $W_{k}(\rho)>\rho W_{k}(1)+(1-\rho) W_{k}(0)$ then $W_{k+1}(\rho)>\rho W_{k+1}(1)+(1-\rho) W_{k+1}(0)$. 
Proof. Note that

$$
\begin{aligned}
W_{k+1}(\rho) & \geqslant W_{k+1}^{\text {sep }}(\rho) \\
& =W_{0}(\rho)+\beta \rho W_{k}(1)+\beta(1-\rho) W_{k}(0) \\
& \geqslant\left[\rho W_{0}(1)+(1-\rho) W_{0}(0)\right]+\beta \rho W_{k}(1)+\beta(1-\rho) W_{k}(0) \\
& =\rho W_{k+1}(1)+(1-\rho) W_{k+1}(0)
\end{aligned}
$$

where the first inequality follows from the definition of $W_{k+1}$ in (48), the second line follows from the definition of $W_{k+1}^{\text {sep }}(\rho)$ in (49), the third line follows from uncertainty being beneficial in the static problem and the induction hypothesis, and the last line follows from the fact that for $\rho \in\{0,1\}, W_{k}(\rho)=W_{k+1}(\rho)=W_{0}(\rho)$. Q.E.D.

The above lemma and the fact that $W_{0}$ satisfies the property implies that for all $k$, $W_{k}(\rho)$ satisfies the above property.

\section{A.7 Proof of Proposition 3}

Let's denote the value of the best sustainable equilibrium with prior $\rho$ by $\bar{W}(\rho)$. Consider first the value when $\rho=0$. Here trigger strategies alone can attain the value of $W_{\text {pool }}$ described above:

$$
\bar{W}(0)=\max _{\pi} w(\phi(\pi), \pi)+\beta \bar{W}(0)
$$

subject to

$$
w(\phi(\pi), \pi) \geqslant(1-\beta) w\left(\phi(\pi), \pi^{*}(\phi(\pi))\right)+\beta \underline{\mathrm{V}}(0)
$$

where the worst equilibrium for the optimizing type is $\underline{V}(0)=W_{0}(0) /(1-\beta)$. Clearly $\bar{W}(0)=W_{\text {pool }}$ and the optimal policy is $\pi_{\mathrm{CK}}$.

Consider now any $\rho>0$. If it is optimal to pool in all periods, the value is

$$
\bar{W}_{\text {pool }}(\rho)=\frac{w\left(\phi\left(\pi_{\mathrm{CK}}\right), \pi_{\mathrm{CK}}\right)}{1-\beta}=\bar{W}(0)=W_{\text {pool }} .
$$

If instead it is optimal to separate in period 0 , the problem solves

$$
\bar{W}_{\text {sep }}(\rho)=\max _{\pi_{\mathrm{c}}, \pi_{0}, x} \rho\left[w\left(x, \pi_{\mathrm{c}}\right)+\beta W_{\text {ramsey }}\right]+(1-\rho)\left[w\left(x, \pi_{\mathrm{o}}\right)+\beta \bar{W}(0)\right]
$$

subject to

$$
\begin{gathered}
x=\phi\left(\rho \pi_{c}+(1-\rho) \pi_{0}\right) \\
w\left(x, \pi_{0}\right)+\beta \bar{V}(0) \geqslant w\left(x, \pi^{*}(x)\right)+\beta \underline{V}(0)
\end{gathered}
$$

and $\pi_{\mathrm{c}} \neq \pi_{\mathrm{o}}$. Note that here $\overline{\mathrm{V}}(0)=w\left(\phi\left(\pi_{\mathrm{CK}}\right), \pi_{\mathrm{CK}}\right) /(1-\beta)$. Clearly, i) $\bar{W}_{\text {sep }}(\rho)>$ 
$W_{\text {sep }}(\rho)$ for all $\rho<1$ where

$$
\begin{aligned}
W_{\text {sep }}(\rho) & =W_{0}(\rho)+\frac{\beta}{1-\beta}\left[\rho W_{0}(1)+(1-\rho) W_{0}(0)\right] \\
& =W_{0}(\rho)+\beta\left[\rho W_{\text {ramsey }}+(1-\rho) \underline{W}(0)\right]
\end{aligned}
$$

and ii) $\bar{W}_{\text {sep }}(0)=\bar{W}(0)=W_{\text {pool }}$ and $\bar{W}_{\text {sep }}(\rho)$ is strictly increasing in $\rho$ so $\bar{W}_{\text {sep }}(\rho)>$ $W_{\text {pool }}$ for all $\rho \in(0,1)$. Thus it is always optimal to separate and the limit of the finite horizon converges to the best sustainable equilibrium only for the trivial case $\rho=1$. Q.E.D.

\section{A.8 Proof of Proposition 4}

We know that when rules are chosen sequentially by the rule designer, following the logic in the text, there exist two cutoffs, $\rho_{1}^{*}$ and $\rho_{2}^{*}$, such that: i) for $\rho>\rho_{1}^{*}$ it is optimal to separate in all periods, ii) for $\rho \in\left(\rho_{2}^{*}, \rho_{1}^{*}\right]$ it is optimal to pool in period 1 but not in period 0 , iii) if $\rho \leqslant \rho_{2}^{*}$ it is optimal to pool in periods 0 and 1 .

Consider now the problem where the rule designer in period 0 can choose the recommended rule for periods 0 and 1 . In particular, consider $\rho \in \rho \in\left(\rho_{2}^{*}, \rho_{1}^{*}\right]$. With commitment, the rule designer in period 0 can choose a stringent rule for period 1 to induce separation in period 1 and therefore relaxing the incentive constraint in period 0 . The value associated with this plan is at least

$$
\begin{aligned}
\tilde{W}_{0}(\rho) & =w\left(x_{1}(\rho), \phi^{-1}\left(x_{1}(\rho)\right)\right)+\beta W_{0}(\rho)+\beta^{2}\left[\rho W_{0}(1)+(1-\rho) W_{0}(0)\right] \\
& \geqslant W_{0}(\rho)+\left(\beta+\beta^{2}\right)\left[\rho W_{0}(1)+(1-\rho) W_{0}(0)\right]
\end{aligned}
$$

where the inequality follows from the fact that for $\rho \leqslant \rho_{1}^{*}$, the value of pooling in the twice repeated economy, $w\left(x_{1}(\rho), \phi^{-1}\left(x_{1}(\rho)\right)\right)+\beta W_{0}(\rho)$, is higher than the value of separation, $W_{0}(\rho)+\beta\left[\rho W_{0}(1)+(1-\rho) W_{0}(0)\right]$.

The other alternative is to choose a rule for period 1 that allows for pooling as in the case without commitment. Since $\rho>\rho_{2}^{*}$, if there is pooling in period 1 then the incentive constraint in period 0 is tight and it is not optimal to pool in period 0 as $\rho_{2}^{*}$ is defined as

$$
\begin{aligned}
& w\left(x_{2}\left(\rho_{2}^{*}\right), \phi^{-1}\left(x_{2}\left(\rho_{2}^{*}\right)\right)\right)+\beta w\left(x_{1}\left(\rho_{2}^{*}\right), \phi^{-1}\left(x_{1}\left(\rho_{2}^{*}\right)\right)\right)+\beta^{2} W_{0}(\rho) \\
& =W_{0}\left(\rho_{2}^{*}\right)+\left(\beta+\beta^{2}\right)\left[\rho_{2}^{*} W_{0}(1)+\left(1-\rho_{2}^{*}\right) W_{0}(0)\right]
\end{aligned}
$$

Thus for all $\rho>\rho_{2}^{*}$ we have that the value of separating is higher than pooling in both 
periods 0 and 1 . Thus the value of this option is

$$
W_{0}(\rho)+\left(\beta+\beta^{2}\right)\left[\rho W_{0}(1)+(1-\rho) W_{0}(0)\right]
$$

which is less than the value $\tilde{W}_{0}(\rho)$ from (62). Hence, it is better to pool in period 0 and induce separation in period 1 for all $\rho \in\left(\rho_{2}^{*}, \rho_{1}^{*}\right]$. Q.E.D.

\section{A.9 Proof of Proposition 7}

We start by proving that for $\rho$ close to 1 it is optimal to have stochastic rules. Consider a $\rho$ close enough to 1 so that with a deterministic rule it is optimal to separate so $\pi=\pi_{0}(\rho)$ . The value of this policy is

$$
\begin{aligned}
W & =\left[\rho w\left(x_{0}, \pi_{0}\right)+(1-\rho) w\left(x_{0}, \pi^{*}\left(x_{0}\right)\right)\right]+\beta\left[\rho W_{0}(1)+(1-\rho) W_{0}(0)\right] \\
& =W_{0}(\rho)+\beta\left[\rho W_{0}(1)+(1-\rho) W_{0}(0)\right]
\end{aligned}
$$

We now show that if $\rho$ is close to 1 then this policy is dominated by one that calls for the commitment type to play the static best response with some positive probability. Consider a deviation indexed by $\varepsilon>0$ sufficiently small so that

$$
\pi_{c}= \begin{cases}\pi_{0}(\rho) & \text { with pr } 1-\varepsilon \\ 1 & \text { with pr } \varepsilon\end{cases}
$$

so after observing a bailout the posterior is

$$
\rho^{\prime}=\frac{\rho \varepsilon}{\rho \varepsilon+(1-\rho)}=\frac{\rho}{\rho+(1-\rho) / \varepsilon}>0
$$

and after no-bailout $\rho^{\prime}=1$. The value of this deviation is then

$$
\begin{aligned}
W^{\operatorname{dev}}(\varepsilon) & =\left[\rho(1-\varepsilon) w\left(x_{0}(\varepsilon), \pi_{0}\right)+[\rho \varepsilon+(1-\rho)] w\left(x_{0}(\varepsilon), \pi^{*}\left(x_{0}\right)\right)\right] \\
& +\beta\left[\rho(1-\varepsilon) W_{0}(1)+[\rho \varepsilon+(1-\rho)] W_{0}\left(\rho^{\prime}\right)\right]
\end{aligned}
$$

Since $W=W^{\text {dev }}(0)$ we have

$$
\begin{aligned}
W^{\mathrm{dev}}(\varepsilon)-W^{\mathrm{dev}}(0) & =\Delta \omega(\varepsilon)+\beta \Delta \Omega(\varepsilon) \\
& \approx\left[\Delta \omega^{\prime}(\varepsilon)+\beta \Delta \Omega^{\prime}(\varepsilon)\right] \varepsilon
\end{aligned}
$$

where

$$
\Delta \omega(\varepsilon)=\left[\rho(1-\varepsilon) \mathcal{w}\left(x_{0}(\varepsilon), \pi_{0}\right)+[\rho \varepsilon+(1-\rho)] \mathcal{w}\left(x_{0}(\varepsilon), \pi^{*}\left(x_{0}\right)\right)\right]
$$




$$
\Delta \Omega(\varepsilon)=\left[\rho(1-\varepsilon) W_{0}(1)+[\rho \varepsilon+(1-\rho)] W_{0}\left(\rho^{\prime}\right)\right]-\left[\rho W_{0}(1)+(1-\rho) W_{0}(0)\right]
$$

Note that

$$
\begin{aligned}
\Delta \Omega^{\prime}(\varepsilon) & =-\rho W_{0}(1)+\rho W_{0}\left(\rho^{\prime}(\varepsilon)\right)+[\rho \varepsilon+(1-\rho)] W_{0}^{\prime}\left(\rho^{\prime}(\varepsilon)\right) \frac{\partial \rho^{\prime}}{\partial \varepsilon} \\
& =-\rho\left[W_{0}(1)-W_{0}\left(\rho^{\prime}(\varepsilon)\right)\right]+[\rho \varepsilon+(1-\rho)] W_{0}^{\prime}\left(\rho^{\prime}(\varepsilon)\right) \frac{\partial \rho^{\prime}}{\partial \varepsilon}
\end{aligned}
$$

As $\varepsilon \rightarrow 0$

$$
\begin{gathered}
\Delta \Omega^{\prime}(\varepsilon) \rightarrow-\rho\left[W_{0}(1)-W_{0}(0)\right]+[(1-\rho)] W_{0}^{\prime}(0) \frac{\partial \rho^{\prime}}{\partial \varepsilon} \\
\frac{\partial \rho^{\prime}}{\partial \varepsilon}=\frac{\rho[\rho \varepsilon+(1-\rho)]-\rho \varepsilon \rho}{[\rho \varepsilon+(1-\rho)]^{2}} \rightarrow \frac{\rho(1-\rho)}{(1-\rho)^{2}}=\frac{\rho}{(1-\rho)}
\end{gathered}
$$

so for $\rho$ close to one

$$
\lim _{\rho \rightarrow 1} \lim _{\varepsilon \rightarrow 0} \Delta \Omega^{\prime}(\varepsilon)=\infty
$$

Thus to show that the deviation is profitable it is sufficient to show that $\Delta \omega>-M$ for some $M$ sufficiently large. Consider

$$
\begin{aligned}
\Delta \omega^{\prime}(\varepsilon) & =\rho\left[w\left(x_{0}(\varepsilon), \pi^{*}\left(x_{0}(\varepsilon)\right)\right)-w\left(x_{0}(\varepsilon), \pi_{0}\right)\right] \\
& +\left\{\rho(1-\varepsilon) w_{x}\left(x_{0}(\varepsilon), \pi_{0}\right)+[\rho \varepsilon+(1-\rho)] w_{x}\left(x_{0}(\varepsilon), \pi^{*}\left(x_{0}\right)\right)\right\} \frac{\partial x_{0}(\varepsilon)}{\partial \varepsilon} \\
& +\rho(1-\varepsilon) w_{\pi}\left(x_{0}(\varepsilon), \pi_{0}\right)
\end{aligned}
$$

where

$$
\frac{\partial x_{0}(\varepsilon)}{\partial \varepsilon}=\phi_{\pi} \rho\left(\pi^{*}-\pi_{0}\right)
$$

Since the first term in square brackets is positive we have that

$$
\begin{aligned}
\Delta \omega^{\prime}(\varepsilon) & >\left\{\rho(1-\varepsilon) w_{x}\left(x_{0}(\varepsilon), \pi_{0}\right)+[\rho \varepsilon+(1-\rho)] w_{x}\left(x_{0}(\varepsilon), \pi^{*}\left(x_{0}\right)\right)\right\} \frac{\partial x_{0}(\varepsilon)}{\partial \varepsilon} \\
& +\rho(1-\varepsilon) w_{\pi}\left(x_{0}(\varepsilon), \pi_{0}\right) \\
& =\left\{\rho w_{x}\left(x_{0}, \pi_{0}\right)+(1-\rho) w_{x}\left(x_{0}, \pi^{*}\left(x_{0}\right)\right)\right\} \phi_{\pi} \rho\left(\pi^{*}-\pi_{0}\right)+\rho w_{\pi}\left(x_{0}, \pi_{0}\right)
\end{aligned}
$$

with $w_{x}$ and $\phi_{\pi}$ bounded, as $\rho \rightarrow 1$ we have that the last expression converges to $\mathcal{w}_{x}\left(x_{0}, \pi_{0}\right) \phi_{\pi}\left(\pi^{*}-\pi_{0}\right)+\mathcal{w}_{\pi}\left(x_{0}, \pi_{0}\right)>\mathcal{w}_{x}\left(x_{0}, \pi_{0}\right) \phi_{\pi}\left(\pi^{*}-\pi_{0}\right)>\mathcal{w}_{x}\left(x_{0}, \pi_{0}\right) \phi_{\pi}(\bar{\pi}-\underline{\pi})>-M$ for some $M<\infty$.

(Notice that to derive this result we are not relying on the concavity of $W_{0}$ but only: $i$ ) $W_{0}^{\prime}>0$ and ii) properties of Bayes' rule.)

We now prove that for $\rho$ close to zero a deterministic rule is optimal. Since $W_{0}$ is 
concave and the posterior is a martingale,

$$
\int \rho^{\prime}(\pi, \rho)\left[\rho \sigma_{\mathcal{c}}(\pi)+(1-\rho) \sigma_{\mathrm{o}}(\pi)\right] \mathrm{d} \pi=\rho,
$$

then

$$
W_{0}(\rho) \geqslant \int W_{0}\left(\rho^{\prime}(\pi, \rho)\right)\left[\rho \sigma_{c}(\pi)+(1-\rho) \sigma_{o}(\pi)\right] d \pi
$$

Thus randomization can be optimal only if it improves that static outcome by reducing $x$. Then it must be that

$$
\mathcal{w}\left(\phi\left(\pi_{\text {ico }}\right), \pi_{\text {ico }}\right)<\int \mathcal{w}(x, \pi)\left[\rho \sigma_{\mathrm{c}}(\pi)+(1-\rho) \sigma_{\mathrm{o}}(\pi)\right] \mathrm{d} \pi
$$

where $x$ is given by (18). A necessary condition is that

$$
x<\phi\left(\pi_{\mathrm{ico}}\right) \Longleftrightarrow \mathbb{E} \pi \equiv \int \pi\left[\rho \sigma_{\mathrm{c}}(\pi)+(1-\rho) \sigma_{\mathrm{o}}(\pi)\right] \mathrm{d} \pi<\pi_{\mathrm{ico}}
$$

Thus, it is sufficient to show that $\mathbb{E} \pi>\pi_{\text {ico }}$ to prove our result. Note that

$$
\mathbb{E} \pi \geqslant \underline{\pi}(\rho)=\min _{\sigma_{\mathcal{c},}, \sigma_{\mathrm{o}} \in \Delta([0,1])} \int \pi\left[\rho \sigma_{\mathcal{c}}(\pi)+(1-\rho) \sigma_{\mathrm{o}}(\pi)\right]
$$

subject to (18), (19), and (20). It is then sufficient to show that $\underline{\pi}(\rho)>\pi_{\text {ico }}(\rho)$ for $\rho$ close to zero. Suppose by way of contradiction that it is not optimal to have $\pi_{\text {ico }}$ with probability 1 so

$$
\int \pi\left[\rho \sigma_{\mathrm{c}}(\pi)+(1-\rho) \sigma_{\mathrm{o}}(\pi)\right] \mathrm{d} \pi<\pi_{\text {ico }}
$$

and since we consider $\rho \rightarrow 0$ then

$$
\mathbb{E}_{\mathrm{o}} \pi=\int \pi \sigma_{\mathrm{o}}(\pi) \mathrm{d} \pi \leqslant \pi_{\mathrm{ico}}
$$

otherwise we can make $\rho$ arbitrary close to 0 so that the inequality in (63) is reversed. From the incentive constraint, it must be that $\forall \pi \in S u p p \sigma_{0}$

$$
w(x, \pi)+\beta \bigvee_{0}\left(\rho^{\prime}(\pi, \rho)\right) \geqslant w\left(x, \pi^{*}(x)\right)+\beta V_{0}\left(\rho^{\prime}\left(\pi^{*}(x), \rho\right)\right) \geqslant w\left(x, \pi^{*}(x)\right)+\beta \bigvee_{0}(0)
$$

where the second inequality follows from $V_{0}$ being increasing in the posterior and $\rho^{\prime}\left(\pi^{*}(x), \rho\right) \geqslant$ 0 . Note now that by properties of Bayes' rule

$$
\int \rho^{\prime}(\pi, \rho) \sigma_{o}(\pi) d \pi=\int \frac{\rho \sigma_{\mathcal{c}}(\pi)}{\rho \sigma_{\mathcal{c}}(\pi)+(1-\rho) \sigma_{o}(\pi)} \sigma_{o}(\pi) d \pi \leqslant \rho .
$$


Thus we have:

$$
\begin{aligned}
\int\left[w(x, \pi)+\beta \mathrm{V}_{0}\left(\rho^{\prime}(\pi, \rho)\right)\right] \sigma_{\mathrm{o}}(\pi) \mathrm{d} \pi & <w\left(x, \mathbb{E}_{\mathrm{o}} \pi\right)+\beta \mathrm{V}_{0}\left(\mathrm{E}_{\mathrm{o}} \rho^{\prime}\right) \\
& \leqslant w\left(x, \mathbb{E}_{0} \pi\right)+\beta \mathrm{V}_{0}(\rho) \\
& =w\left(\phi\left(\mathbb{E}_{0} \pi\right), \mathbb{E}_{\mathrm{o}} \pi\right)+\beta \mathrm{V}_{0}(\rho)
\end{aligned}
$$

where the first inequality follows from the strict concavity of $w$ (in $\pi$ ) and $V_{0}$, the second inequality from (66) and $V_{0}$ strictly increasing. Thus combining (65) and (67) we have that

$$
w\left(\phi\left(\mathbb{E}_{0} \pi\right), \mathbb{E}_{0} \pi\right)+\beta \bigvee_{0}(\rho)>w\left(\phi\left(\mathbb{E}_{0} \pi\right), \pi^{*}\left(\phi\left(\mathbb{E}_{0} \pi\right)\right)\right)+\beta \bigvee_{0}(0)
$$

Since $\pi_{\text {ico }}$ is the smallest solution to

$$
w(\phi(\pi), \pi)+\beta V_{0}(\rho)=w\left(\phi(\pi), \pi^{*}(\pi)\right)+\beta V_{0}(0)
$$

then it follows that for $\rho$ close to zero

$$
\pi_{\text {ico }}(\rho)<\mathbb{E}_{0} \pi
$$

a contradiction. Q.E.D.

\section{A.10 Proof of Proposition 8}

We consider the case with $w_{x}<0$. Note that the statically optimal rule chosen by the commitment type is $\underline{\pi}$. To see why note that the first order condition for the commitment type is

$$
\begin{aligned}
& w_{\pi}(x, \pi)+w_{x}(x, \pi) \frac{\phi^{\prime}(\cdot)}{\left[1-\phi^{\prime}(\cdot)(1-\rho) \pi_{x}^{*}(x)\right]} \\
& \leqslant w_{\pi}(x, \pi)+\left[\rho w_{x}(x, \pi)+(1-\rho) w_{x}\left(x, \pi^{*}(x)\right) \frac{\phi^{\prime}(\cdot)}{\left[1-\phi^{\prime}(\cdot)(1-\rho) \pi_{x}^{*}(x)\right]}\right. \\
& \leqslant 0
\end{aligned}
$$

where the first inequality follows from the assumption that $w_{x \pi} \geqslant 0$ and the last inequality from Assumption 2. Let

$$
\mathrm{V}_{0}^{\mathrm{c}}(\rho)=w\left(\mathrm{x}_{0}(\rho), \underline{\pi}\right),
$$

be the value for the commitment type in the terminal period given the prior $\rho$ where

$$
x_{0}(\rho)=\phi\left(\rho \underline{\pi}+(1-\rho) \pi^{*}\left(x_{0}(\rho)\right)\right) .
$$


We can write the value for the commitment type if it chooses to separate as

$$
V_{\text {sep }}^{c}(\rho)=w\left(x_{0}(\rho), \underline{\pi}\right)+\beta V_{0}^{c}(1),
$$

while the value of pooling is

$$
\mathrm{V}_{\text {pool }}^{\mathrm{c}}(\rho)=w\left(\phi\left(\pi_{\text {ico }}(\rho)\right), \pi_{\text {ico }}(\rho)\right)+\beta \mathrm{V}_{0}^{\mathrm{c}}(\rho)
$$

where $\pi_{\text {ico }}$ solves

$$
w\left(\phi\left(\pi_{\text {ico }}(\rho)\right), \pi_{\text {ico }}(\rho)\right)+\beta V_{0}(\rho)=w\left(\phi\left(\pi_{\text {ico }}(\rho)\right), \pi^{*}\left(\phi\left(\pi_{\text {ico }}(\rho)\right)\right)\right)+\beta V_{0}(0)
$$

Therefore,

$$
V_{\text {sep }}^{c}(\rho)-V_{\text {pool }}^{c}(\rho)=\left[w\left(x_{0}(\rho), \underline{\pi}\right)-w\left(\phi\left(\pi_{\text {ico }}\right), \pi_{\text {ico }}\right)\right]+\beta\left[V_{0}^{c}(1)-V_{0}^{c}(\rho)\right]
$$

First note that for $\rho$ sufficiently large separating has both dynamic gains, $V_{0}^{c}(1)-V_{0}^{c}(\rho)>$ 0 , and static gains as $\left[w\left(x_{0}(\rho), \underline{\pi}\right)-w\left(\phi\left(\pi_{i c o}\right), \pi_{\text {ico }}\right)\right]>0$. In particular, for $\rho \rightarrow 1$ we have that the static gains of separating converge to

$$
\left[w(\phi(\underline{\pi}), \underline{\pi})-w\left(\phi\left(\pi_{\mathrm{ico}}(1)\right), \pi_{\mathrm{ico}}(1)\right)\right]
$$

which is positive since under our assumption thatthe Ramsey outcome is not sustainable, $\pi_{\text {ico }}(\rho)<\underline{\pi}$. Consequently, for $\rho$ large enough the commitment type will choose a stringent rule and thus there will be separation.

Next, given some $\rho$, the commitment type would like to separate if

$$
\beta \geqslant \underline{\beta}(\rho) \equiv \frac{\left[w\left(\phi\left(\pi_{\text {ico }}(\rho)\right), \pi_{\text {ico }}(\rho)\right)-w\left(x_{0}(\rho), \underline{\pi}\right)\right]}{\left[V_{0}^{c}(1)-V_{0}^{c}(\rho)\right]}
$$

To show that it is optimal for the optimizing type to separate at $\underline{\pi}$ it must be that

$$
w\left(x_{0}(\rho), \pi^{*}\left(x_{0}(\rho)\right)\right)+\beta V_{0}(0)>w\left(x_{0}(\rho), \underline{\pi}\right)+\beta V_{0}(1)
$$

(note that if the optimizing type mimics the commitment type the posterior jumps to one because we are constructing an equilibrium with separation) or

$$
\beta<\bar{\beta}(\rho) \equiv \frac{\left[w\left(x_{0}(\rho), \pi^{*}\left(x_{0}(\rho)\right)\right)-w\left(x_{0}(\rho), \underline{\pi}\right)\right]}{V_{0}(1)-V_{0}(0)}
$$


Therefore, the equilibrium outcome of the signaling game has separation if

$$
\bar{\beta}(\rho)>\beta>\underline{\beta}(\rho)
$$

Thus we need to show that such an interval exists. For $\rho \rightarrow 0$ we have

$$
\begin{aligned}
& \lim _{\rho \rightarrow 0} \bar{\beta}(\rho)=\frac{w\left(x_{0}(0), \pi^{*}\left(x_{0}(0)\right)\right)-w\left(x_{0}(0), \underline{\pi}\right)}{V_{0}(1)-V_{0}(0)} \\
& \lim _{\rho \rightarrow 0} \underline{\beta}(\rho)=\frac{w\left(x_{0}(0), \pi^{*}\left(x_{0}(0)\right)\right)-w\left(x_{0}(0), \underline{\pi}\right)}{V_{0}^{c}(1)-V_{0}^{c}(0)}
\end{aligned}
$$

since $\pi_{\text {ico }}(\rho) \rightarrow \pi^{*}\left(x_{0}(0)\right)$. Thus to compare $\bar{\beta}(0)$ and $\underline{\beta}(0)$ we only need to compare the denominators since the numerators are identical. In particular, $\bar{\beta}(0)>\beta(0)$ if and only if $\mathrm{V}_{0}(1)-\mathrm{V}_{0}(0)<\mathrm{V}_{0}^{\mathrm{c}}(1)-\mathrm{V}_{0}^{\mathrm{c}}(0)$, or

$$
w\left(x_{0}(1), \pi^{*}\left(x_{0}(1)\right)\right)-w\left(x_{0}(0), \pi^{*}\left(x_{0}(0)\right)\right)<w\left(x_{0}(1), \underline{\pi}\right)-w\left(x_{0}(0), \underline{\pi}\right)
$$

or

$$
w\left(x_{0}(1), \pi^{*}\left(x_{0}(1)\right)\right)-w\left(x_{0}(1), \underline{\pi}\right)<w\left(x_{0}(0), \pi^{*}\left(x_{0}(0)\right)\right)-w\left(x_{0}(0), \underline{\pi}\right)
$$

Note that

$$
w\left(\mathrm{x}_{0}(0), \pi^{*}\left(\mathrm{x}_{0}(0)\right)\right)-w\left(\mathrm{x}_{0}(0), \underline{\pi}\right) \geqslant w\left(\mathrm{x}_{0}(0), \pi^{*}\left(\mathrm{x}_{0}(1)\right)\right)-w\left(\mathrm{x}_{0}(0), \underline{\pi}\right)
$$

so we are left to show that

$$
w\left(\mathrm{x}_{0}(0), \pi^{*}\left(\mathrm{x}_{0}(1)\right)\right)-w\left(\mathrm{x}_{0}(0), \underline{\pi}\right)>w\left(\mathrm{x}_{0}(1), \pi^{*}\left(\mathrm{x}_{0}(1)\right)\right)-w\left(\mathrm{x}_{0}(1), \underline{\pi}\right)
$$

Under Assumption 1, for $x_{H}>x_{L}$

$$
\int_{\left[\underline{\pi} \pi^{*}\right]} w_{\pi}\left(x_{H}, \pi\right) d \pi>\int_{\left[\underline{\pi}, \pi^{*}\right]} w_{\pi}\left(x_{L}, \pi\right) d \pi
$$

Thus, since $x_{0}(0)>x_{0}(1)$ the inequality is satisfied. Q.E.D.

\section{B Example where uncertainty is not beneficial}

Here we present an example of an economy where uncertainty is not beneficial. This economy is similar to our bailout example but there is no effort choice.

There are two types of private agents: depositors and bankers. At the beginning of each period the banker has no capital and must borrow $k=1$ from the depositors to 
finance an investment opportunity that pays off at the end of the period. The return of the investment opportunity is $R_{H}$ with probability $p$ and 0 with probability $1-p$. The banker offers a contract to depositors that promises to repay $R$ units of the consumption good in the second sub-period subject to limited liability. We assume that there are bankruptcy costs $\psi$ in case of a default. The policy maker can avoid this bankruptcy costs by making a transfer to the banker. In particular, the policy maker can choose the recovery rate $\pi$ in case of inability of the banker to repay. With a recovery rate $\pi$, the bankruptcy costs are $\psi(1-\pi)$.

The rule designer and the policy makers care about the depositor's utility net of bankruptcy costs.

Depositors are then willing to lend to the banker at an interest rate

$$
\mathrm{Q}(\mathrm{b}, \mathbb{E} \pi)=[\mathrm{p}+(1-\mathrm{p}) \mathbb{E} \pi]
$$

so the banker's problem is

$$
\max _{b} p \max \left\{R_{H}-b, 0\right\}
$$

subject to

$$
1=\mathrm{Q}(\mathrm{b}, \mathbb{E} \pi) \mathrm{b} .
$$

In equilibrium, it must be that $b=1 / Q(b, \mathbb{E} \pi)$ so we can let $x=Q$ with

$$
\mathrm{Q}=\phi(\mathbb{E} \pi)=[\mathrm{p}+(1-\mathrm{p}) \mathbb{E} \pi]
$$

and express the social welfare function as

$$
\mathcal{w}(\mathrm{Q}, \pi)=-1+\mathrm{p} \frac{1}{\mathrm{Q}}-(1-\mathrm{p}) \psi \frac{1}{\mathrm{Q}} \max \{1-\pi, 0\}
$$

We first show that the solution to the static rule designer's value is convex in $\rho$ :

Lemma 8. If $p>1 / 2$, the static rule designer's value $W_{0}(\rho)$ is convex.

Proof. Consider

$$
W_{0}(\rho)=\max _{\pi_{c}, Q}-\left[1-p \frac{1}{Q}\right]-(1-p) \rho \psi \frac{1}{Q} \max \left\{1-\pi_{c}, 0\right\}
$$

subject to (68) or, using the constraint to substitute for Q as

$$
W_{0}(\rho)=\max _{\pi_{c}}-1+\frac{\left[p-(1-p) \rho \psi\left(1-\pi_{c}\right)\right]}{\left[p+(1-p)\left(\rho \pi_{c}+(1-\rho)\right)\right]}
$$


Differentiating with respect to $\pi_{\mathrm{c}}$ we obtain

$$
\frac{(1-p) \rho \psi\left[p+(1-p)\left(\rho \pi_{c}+(1-\rho)\right)\right]-(1-p) \rho\left[p-(1-p) \rho \psi\left(1-\pi_{c}\right)\right]}{\left[p+(1-p)\left(\rho \pi_{c}+(1-\rho)\right)\right]^{2}}
$$

which is negative if $\psi$ is sufficiently small. So the optimal static rule is $\pi_{c}=0$ for all $\rho$. Thus we have that

$$
W_{0}(\rho)=\frac{[p-(1-p) \rho \psi]}{p-\rho(1-p)}-1
$$

which is convex as

$$
\begin{aligned}
W_{0}^{\prime}(\rho) & =\frac{-(1-p) \psi[p-\rho(1-p)]+(1-p)[p-(1-p) \rho \psi]}{[p-\rho(1-p)]^{2}} \\
& =\frac{(1-p) p(1-\psi)}{[p-\rho(1-p)]^{2}}
\end{aligned}
$$

and

$$
W_{0}^{\prime \prime}(\rho)=2(1-p) \frac{(1-p) p(1-\psi)}{[p-\rho(1-p)]^{3}}=2(1-p) \frac{(1-p) p(1-\psi)}{[p(1+\rho)-\rho]^{3}}>0
$$

as long as $p>1 / 2$. Q.E.D.

Consider now the dynamic problem (twice repeated) in (8). Because of the convexity of $W_{0}(\rho)$, the dynamic benefits of pooling, $\Delta \Omega(\rho)=W_{0}(\rho)-\left[\rho W_{0}(1)+(1-\rho) W_{0}(0)\right]$, are negative. The static benefits of pooling, $\Delta \omega$, are positive for low levels of reputation and negative for high levels. If $\beta$ is sufficiently high we have that $\Delta \omega(\rho)+\beta \Delta \Omega(\rho)<0$ and so we have the following counterpart of Proposition 1:

Proposition 9. In this example where condition (5) does not hold and uncertainty is welfare reducing, under Assumptions 1 and 3, if $\beta$ is sufficiently large then for all $\rho$ there is separation with probability 1 and $\pi=\pi_{0}$. 